\title{
$N$-Glycosylation with Sulfoxide Donors for the Synthesis of Peptidonucleosides
}

\author{
This article is dedicated to the memory of our esteemed colleague Dr. Mazen Es-Sayed
}

Margaux Beretta, ${ }^{\mathrm{a}}$ Emilie Rouchaud, ${ }^{\mathrm{a}}$ Lionel Nicolas, ${ }^{\mathrm{b}}$ Jean-Pierre Vors, ${ }^{\mathrm{b}}$ Thomas Dröge, ${ }^{\mathrm{c}}$ Mazen Es-Sayed, ${ }^{\mathrm{c}}$ JeanMarie Beau, *a,d and Stéphanie Norsikian *a

\footnotetext{
a. Université Paris-Saclay, CNRS, Institut de Chimie des Substances Naturelles, UPR 2301, 91198, Gif-sur-Yvette, France..

b. Bayer Cropscience, Disease Control Chemistry, Centre de Recherche de La Dargoire, 14 impasse Pierre Baizet, 69009 Lyon, France

c. Bayer CropScience, Disease Control Chemistry, Alfred-Nobel-Str. 50, 40789 Monheim am Rhein, Germany

d.Institut de Chimie Moléculaire et des Matériaux d'Orsay (ICMMO), UMR 8182, Univ. Paris-Sud and CNRS, Université Paris-Saclay, F91405 Orsay, France
}

\begin{abstract}
We report here the synthesis of peptidonucleosides obtained after glycosylation of different pyrimidine bases with glucopyranosyl donors carrying an azide group at the $\mathrm{C} 4$ position. A methodological study involving different anomeric leaving groups (acetate, phenylsulfoxide and ortho-hexynylbenzoate) showed that a sulfoxide donor in combination with trimethylsilyl triflate as the promoter led to the best yields.
\end{abstract}

Fungicides represent a class of pesticides used to prevent the development of a fungus in the plant and / or to fight a fungal infection caused by a pathogen. ${ }^{1}$ Their use has become essential over time to avoid contamination of whole crops and improve agricultural yields in a context of increasing urbanization that continues to decrease the area of cultivable land. ${ }^{2}$ The continuous use of agrochemicals has led to the development of pest resistance to active substances, which makes some pesticides ineffective. ${ }^{3}$ Thus, finding new structural motifs with new antifungal modes of action to fight against pathogens is essential to circumvent resistances. Natural products remain an important source of inspiration for the discovery of new active molecules. Among them gougerotin, isolated for the first time in 1962 from strains of Streptomyces gougerotii, ${ }^{4}$ caught the attention of researchers. ${ }^{5}$ This peptidylnucleoside consists of a glucan-type pyranose saccharide motif that has a carboxamide group at the 6-position. It is also substituted at the 4position by a dipeptide made of $\mathrm{D}$-serine and sarcosine and $N$-linked at the anomeric position by a cytosine base. Since its discovery, gougerotin has been the subject of three total syntheses, including two described in the $1970 \mathrm{~s}^{6}$ and a more recent one in 2005 using solid- and solution-phase methodology. ${ }^{7}$ Gougerotin has a very broad spectrum of biological activities: antiviral, ${ }^{8}$ antifungal, ${ }^{5}$ antiparasitic and antibacterial ${ }^{9}$ by inhibiting protein synthesis in procaryotic and eucaryotic systems. It is active on various varieties of plants whether in preventive or curative tests but its phytotoxicity limits its direct use on plants. ${ }^{10}$ In order to optimize its crop specificity, we were interested in the preparation of a few gougerotin analogues. The main modifications will concern the replacement of the natural nucleic base by other pyrimidine bases while preserving the glucopyranosyl skeleton of the parent molecule (Figure 1). The carboxamide function at C5 will also be replaced by a free hydroxymethyl group. To access these compounds, different bases will first be glycosylated with a donor carrying an azide group at the $\mathrm{C} 4$ position. Following this glycosylation step, the azide will be reduced to the corresponding amine allowing a dipeptide coupling. A potential impact of the dipeptide motif on the bioactivity will also be studied with the synthesis of compounds comprising Lserine instead of D-serine or a modified peptide.

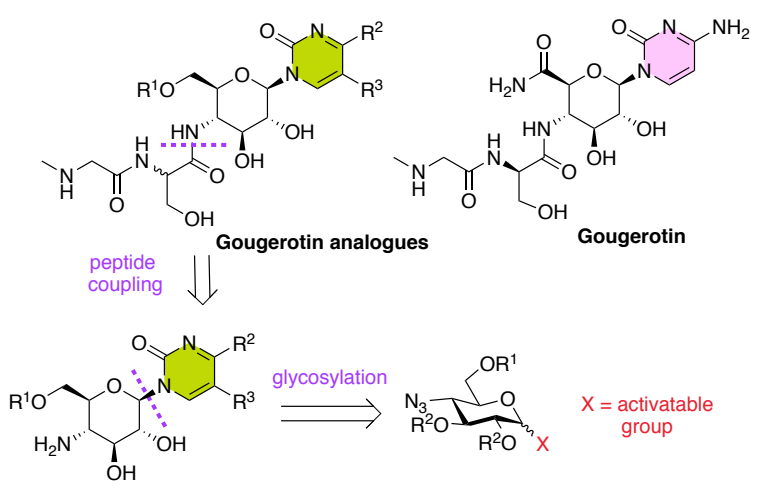

Figure 1. Retrosynthetic scheme of gougerotin analogues 
First of all, we prepared anomeric acetate donor 5, which is the most conventionally activatable donor used to synthesize nucleoside derivatives under Vorbrüggen glycosylation conditions (Scheme 1). ${ }^{11}$ Methyl $\alpha$-Dgalactopyranoside 1 a was first protected as a 4,6-benzylidene acetal to yield the corresponding product, which was esterified with acetic anhydride in pyridine. Compound $\mathbf{2 a}$ was then engaged in a reductive opening reaction of the 4,6-O-benzylidene acetal to lead to galactopyranoside 3a having a free hydroxyl at position 4 . Best results for this step were obtained using triethylsilane ( 2.5 eq.) in combination with triflic acid (2 eq.) as described by Sakagami and Hamana. ${ }^{12}$ Since we also observed the formation of the corresponding 4- $O$-triethylsilyl ether adduct, the reaction mixture was further treated with tetrafluoroboric acid to furnish the desired alcohol 3a in $63 \%$ yield. The latter was then reacted with $\mathrm{Tf}_{2} \mathrm{O}$ in the presence of pyridine to form the triflate in the 4-position, that was displaced by $\mathrm{NaN}_{3}$ with inversion of configuration leading to the glucopyranoside adduct $\mathbf{4 a}$. A final acetolysis step led to donor $\mathbf{5}$ as a mixture of anomers $(\alpha / \beta=8: 2)$.

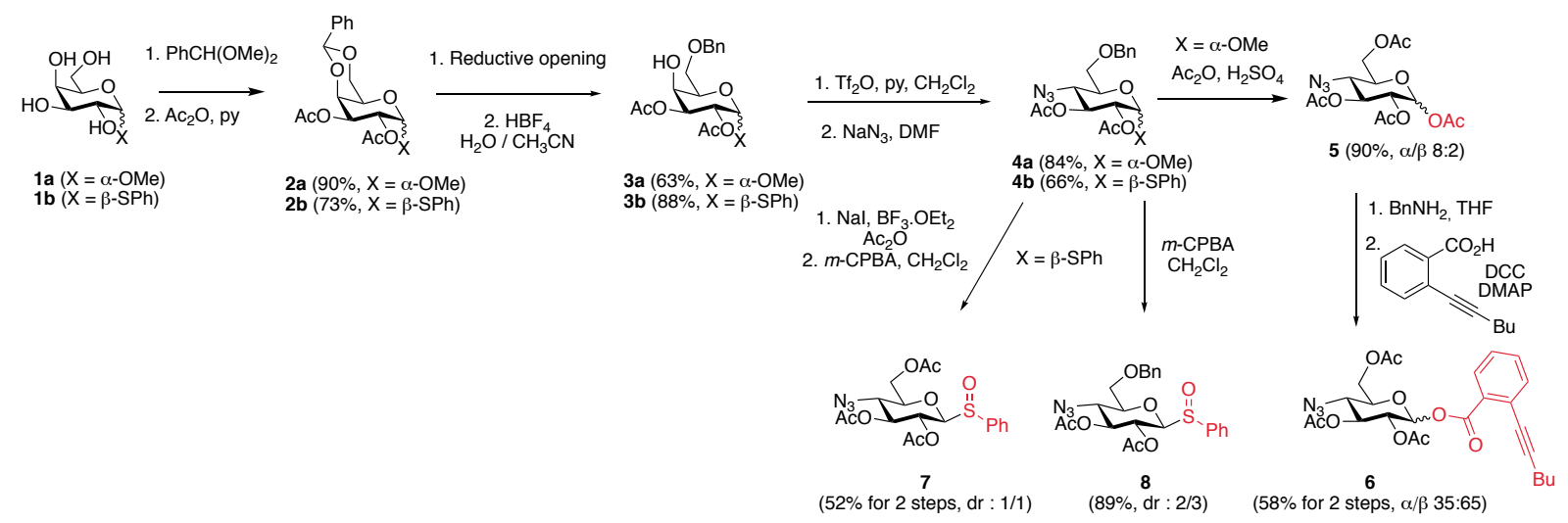

Scheme 1. Preparation of different donors 5-8. Conditions for the reductive opening: with $\mathbf{2 a}(\mathrm{X}=\alpha-\mathrm{OMe}) \mathrm{Et} \mathrm{t}_{3} \mathrm{SiH}\left(2.5 \mathrm{eq}\right.$.), $\mathrm{TfOH}\left(2 \mathrm{eq}\right.$.) in $\mathrm{CH}_{3} \mathrm{CN}$, with $\mathbf{2 b}(\mathrm{X}=\beta$ $\mathrm{SPh}) \mathrm{Et}_{3} \mathrm{SiH}$ (1 eq.), cat. $\mathrm{Cu}(\mathrm{OTf})_{2}$ in $\mathrm{CH}_{3} \mathrm{CN}$

With this donor in hand, we first performed the glycosylation of $\mathrm{N}^{4}$-Ac-cytosine 9 , the nucleic base naturally present in the gougerotin. Reaction with $N, O$-bis(trimethylsilyl)acetamide (BSA) in MeCN gave the corresponding silylated cytosine, which was subsequently treated with $\mathbf{5}$ in the presence of trimethylsilyl triflate (TMSOTf, 1.5 eq.) for $12 \mathrm{~h}$ at $55{ }^{\circ} \mathrm{C} .{ }^{13}$ After workup and purification, the nucleoside 15 was isolated in $36 \%$ yield (Scheme 2, Table 1, entry 1 ) and as a single $\beta$-anomer due to the anchimeric assistance of the 2-OAc group. This was confirmed by the ${ }^{1} \mathrm{H}$ NMR spectrum showing a large coupling constant between protons $\mathrm{H}-1$ and $\mathrm{H}-2\left({ }^{3} J_{1,2}=9.5 \mathrm{~Hz}\right)$ indicating an axial orientation of both protons. The yield could be increased to $69 \%$ by using the Vorbrüggen method with $\mathrm{SnCl}_{4}$ ( 3 eq.) in $\mathrm{MeCN}$ at $55^{\circ} \mathrm{C}$ for $12 \mathrm{~h}$ (Table 1, entry 2). ${ }^{14}$ The glycosylation of other pyrimidine bases was then tested and for each base, the reaction conditions had to be readjusted accordingly. The results are summarized in Table 1. Similar yields were obtained with thymine $\mathbf{1 0}$ (entry 3) and 5-F-uracil 11 (entry 4), which led respectively to the $\beta$ glycosylated adducts $\mathbf{1 6}$ and $\mathbf{1 7}$ in 74 and $71 \%$ yield respectively. Note that 5-F-uracil 11 was silylated in the presence of 1,1,1,3,3,3-hexamethyldisilazane (HMDS) in combination with saccharin (5 mol\%) as catalyst. ${ }^{15}$ With 5 -Me- $\mathrm{N}^{4}$ obtained with silylated uracil 14, which led to the corresponding $\beta$-nucleoside 20 (entry 7). This disappointing result led us to consider the use of other donors for these glycosylations. We started our investigations with the preparation of glycosyl ortho-hexynyl benzoate $\mathbf{6}$, readily accessible from $\mathbf{5}$ after selective deacetylation at the anomeric position and subsequent esterification of the corresponding hemiacetal (Scheme 1). Recently, these donors have been shown to be superior donors for the $N$-glycosylation of nucleobases using gold catalysis under very mild conditions. ${ }^{16}$ Donor 6 was then subjected to glycosylation with silylated pyrimidine bases under the catalysis of $\mathrm{Ph}_{3} \mathrm{PAuNTf}_{2}(10 \mathrm{~mol} \%)$ Bz-cytosine 12 (entry 5) and 5-F-N - -Bz-cytosine $\mathbf{1 3}$ (entry 6), which are rarely glycosylated with pyranosyl donors, moderate yields of 45 and $51 \%$ were achieved respectively for $\mathbf{1 8}$ and $\mathbf{1 9}$. A much lower yield of $25 \%$ was also at room temperature in $\mathrm{MeCN}$. In all cases the corresponding nucleosides were isolated with an excellent $\beta$-selectivity but with variable yields depending on the acceptor. For example, a good 78\% yield was obtained with uracil 14 (entry 13). Moderate results (30 to 49\%) were achieved with other bases (entries 8-11) and, with 5-F- $\mathrm{N}^{4}-\mathrm{Bz}$ cytosine 13, the 

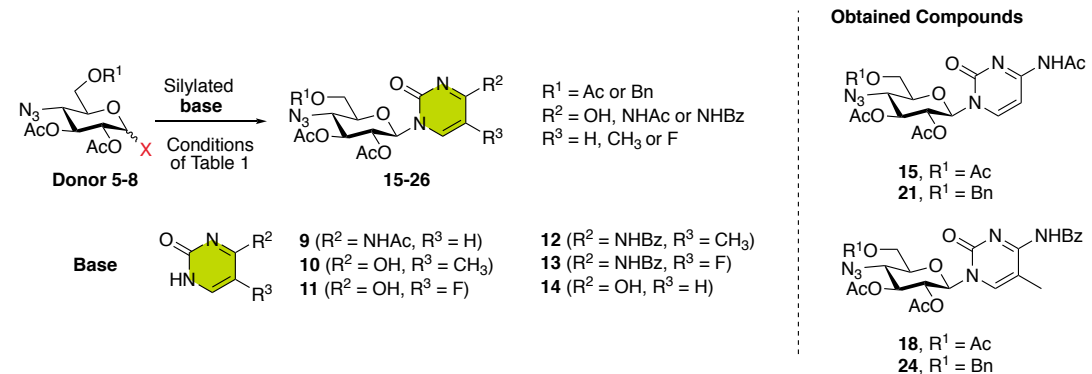

Scheme 2. Glycosylation of different pyrimidine bases 9-14 using donors 5-8

\begin{tabular}{|c|c|c|c|c|}
\hline entry & Donor & Base $^{a}$ & Conditions ${ }^{\mathrm{b}}$ & $\begin{array}{c}\text { Compound, } \\
\text { Yield (\%) }\end{array}$ \\
\hline 1 & 5 & 9 & TMSOTf (1.5 eq.) & $\mathbf{1 5}, 36$ \\
\hline 2 & 5 & 9 & $\mathrm{SnCl}_{4}(3 \text { eq. })^{\mathrm{c}}$ & $\mathbf{1 5}, 69$ \\
\hline 3 & 5 & 10 & $\mathrm{SnCl}_{4}$ (3 eq.) & 16,74 \\
\hline 4 & 5 & $11^{\mathrm{d}}$ & TMSOTf (1.5 eq.) & 17,71 \\
\hline 5 & 5 & 12 & $\mathrm{SnCl}_{4}$ (3 eq.) & 18,45 \\
\hline 6 & 5 & 13 & $\mathrm{SnCl}_{4}$ (3 eq.) & 19,51 \\
\hline 7 & 5 & $14^{\mathrm{d}}$ & TMSOTf (1.5 eq.) & $\mathbf{2 0}, 25$ \\
\hline 8 & 6 & $9^{e, f}$ & $\mathrm{Ph}_{3} \mathrm{PAuNTf}_{2}(10 \mathrm{~mol} \%)$ & $\mathbf{1 5}, 49$ \\
\hline 9 & 6 & $10^{\mathrm{e}, \mathrm{f}}$ & $\mathrm{Ph}_{3} \mathrm{PAuNTf}_{2}(10 \mathrm{~mol} \%)$ & $\mathbf{1 6}, 48$ \\
\hline 10 & 6 & $11^{\mathrm{f}}$ & $\mathrm{Ph}_{3} \mathrm{PAuNTf}_{2}(10 \mathrm{~mol} \%)$ & 17,40 \\
\hline 11 & 6 & $12^{\mathrm{e}, \mathrm{f}}$ & $\mathrm{Ph}_{3} \mathrm{PAuNTf}_{2}(10 \mathrm{~mol} \%)$ & 18,30 \\
\hline 12 & 6 & $13^{\mathrm{f}}$ & $\mathrm{Ph}_{3} \mathrm{PAuNTf}_{2}(10 \mathrm{~mol} \%)$ & 19,9 \\
\hline 13 & 6 & $14^{\mathrm{e}, \mathrm{f}}$ & $\mathrm{Ph}_{3} \mathrm{PAuNTf}_{2}(10 \mathrm{~mol} \%)$ & 20,78 \\
\hline 14 & 7 & 9 & TMSOTf (1.5 eq.) & $\mathbf{1 5}, 95$ \\
\hline 15 & 7 & 10 & TMSOTf (1.5 eq.) & 16,89 \\
\hline 16 & 7 & 11 & TMSOTf (1.5 eq.) & $\mathbf{1 7}, 71$ \\
\hline 17 & 7 & 12 & TMSOTf (1.5 eq.) & 18,88 \\
\hline 18 & 7 & 13 & TMSOTf (1.5 eq.) & 19,75 \\
\hline 19 & 7 & 14 & TMSOTf (1.5 eq.) & $\mathbf{2 0}, 94$ \\
\hline 20 & 8 & 9 & TMSOTf (1.5 eq.) & 21,54 \\
\hline 21 & 8 & 10 & TMSOTf (1.5 eq.) & 22,77 \\
\hline 22 & 8 & $11^{\mathrm{d}}$ & TMSOTf (1.5 eq.) & 23,76 \\
\hline 23 & 8 & 12 & TMSOTf (1.5 eq.) & $\mathbf{2 4}, 66$ \\
\hline 24 & 8 & 13 & TMSOTf (1.5 eq.) & $\mathbf{2 5}, 50$ \\
\hline 25 & 8 & 14 & TMSOTf (1.5 eq.) & $\mathbf{2 6}, 81$ \\
\hline
\end{tabular}

a) BSA (4 eq.) was used as silylating agent unless otherwise stated. b) the reaction mixture was heated for $12 \mathrm{~h}$ at $55{ }^{\circ} \mathrm{C}$ in $\mathrm{MeCN}$ unless otherwise stated c) the reaction was carried out in DCE. d) HMDS (1.8 eq.), saccharine (5 mol.\%) e) with BSTFA ( $N, O$-bis(trimethylsilyl)trifluoroacetamide). f) the reaction was carried out for $48 \mathrm{~h}$ at r.t.

reaction led to a low conversion with the desired $\beta$-glycosylated adduct 19 been isolated in only $9 \%$ yield (entry 12 ). We then turned our attention to the use of sulfoxide donors ${ }^{17}$ that we recently exploited with success in problematic glycosylations ${ }^{18}$ and which have been very rarely used for the $N$-glycosylation with furanosyl or pyranosyl derivatives. ${ }^{19}$ Donor 7 was prepared from phenyl $\beta$-D-thiogalactoside $\mathbf{1 b}$, following the same strategy as for $\mathbf{5}$, i.e. 4,6-O-benzylidene acetal formation, $O-2 / O-3$ acetylation (to $2 \mathbf{b}$ ), reductive opening of the acetal (Et $\mathrm{SiH}_{3}$ with cat. $\mathrm{Cu}(\mathrm{OTf})_{2}$ to $\left.\mathbf{3 b}\right)^{20}$ and introduction of the azide at C-4 through triflate displacement to afford $\mathbf{4 b}$ (Scheme 1). The obtained $\mathbf{4 b}$ was further treated by $\mathrm{NaI} / \mathrm{BF}_{3} \cdot \mathrm{OEt}_{2}$ in acetic anhydride leading to the acetolysis of the $6-\mathrm{OBn}$ position. ${ }^{21}$ Thioether oxidation was carried out under common conditions ( $m$-CPBA) smoothly giving sulfoxides 7 as a mixture of two diastereomers (dr of 1:1). Donor 7 was then subjected to glycosylation after prior silylation of the pyrimidine bases with BSA in MeCN. In all cases, TMSOTf (1.5 eq.) was used as promoter and after $12 \mathrm{~h}$ at $55^{\circ} \mathrm{C}$, we were pleased to obtain the corresponding $\beta$-nucleosides in good to excellent yields ranging from 71 to $95 \%$ (entries 14-19). We also prepared 6-OBn sulfoxide derivatives $\mathbf{8}$ (dr of 2:3) by direct oxidation of $\mathbf{4 b}$. $N$-glycosylation with this donor 8 efficiently led to 6-OBn $\beta$-nucleosides in comparable yields (50-81\%, entries 20-25).

In order to carry out the peptide coupling and access analogues, we first examined azide reduction directly on compound 15. However, while using Staudinger conditions ${ }^{22}$ or different hydrogenation conditions to reduce the azide 

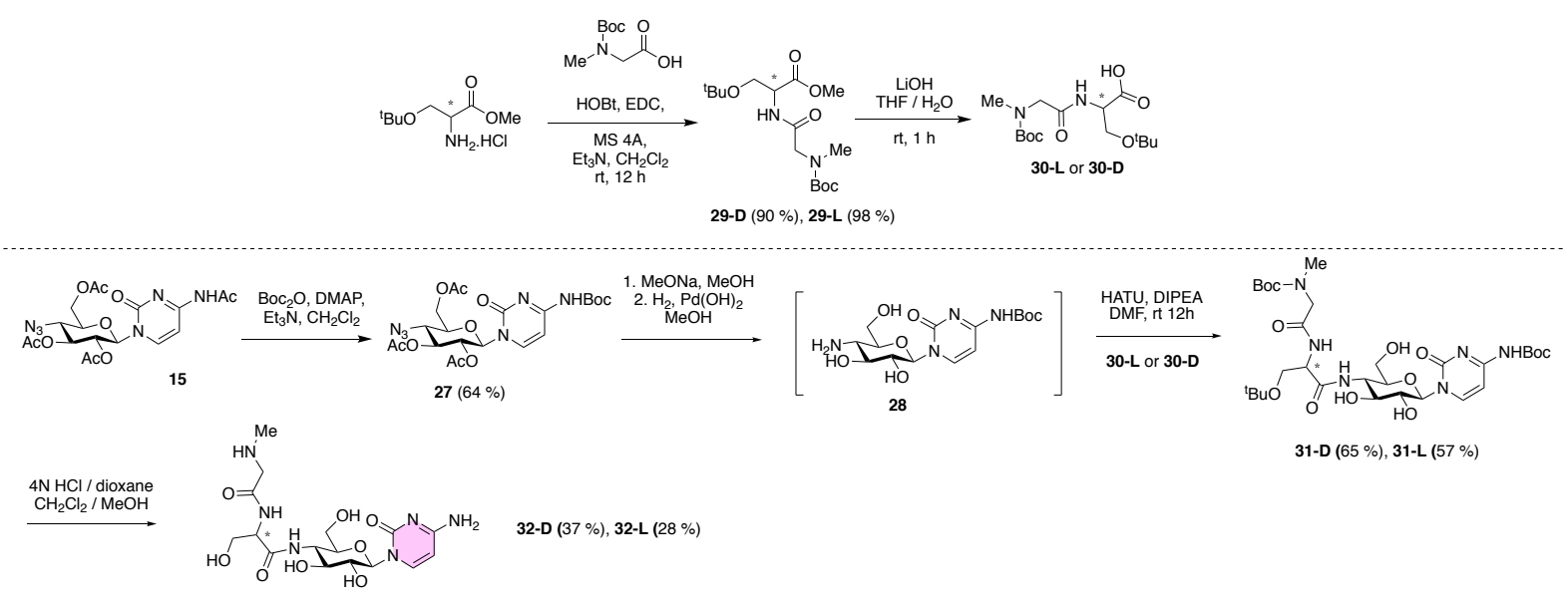

Scheme 3. Preparation of analogues 32-D and 32-L

to amine, we obtained complex mixtures that probably result from deacetylation and acetate migration. To solve this problem, we decided to protect first the $\mathrm{N}^{4}$-cytosine with a tert-butylcarbamate group and remove all the acetyl groups before hydrogenation (Scheme 3). Therefore, compound 15 was treated with $\mathrm{Boc}_{2} \mathrm{O}$ in the presence of NEt 3 and 4dimethylaminopyridine (DMAP) to provide the corresponding derivative 27 by concomitant deprotection of $\mathrm{N}^{4}$-Accytosine. Zemplén deacetylation was carried out followed by hydrogenation with palladium hydroxide in methanol. This sequence led to the corresponding amine $\mathbf{2 8}$ that was used without purification in the following peptide coupling. The synthesis of the dipeptide fragment 30-D, naturally present in the gougerotin, started with the coupling of the methyl ester of D-O-tert-butyl-serine with Boc-sarcosine using hydroxybenzotriazole (HOBt) and 1-ethyl-3-(3dimethylaminopropyl)carbodiimide (EDC) in the presence of triethylamine in $\mathrm{CH}_{2} \mathrm{Cl}_{2}$. The obtained dipeptide 29-D was then saponified with lithium hydroxide to carboxylic acid 30-D. The same sequence was also performed with the methyl ester of L-O-tert-butyl-serine providing the corresponding dipeptide fragment 30-L. The peptide coupling of amine 28 was then carried out with both dipeptides 30-D and 30-L using the coupling agent HATU ${ }^{23}$ in the presence of Hünig's base in DMF to furnish 31-D and 31-L in 65 and 57\% yield respectively. Both compounds were then treated with $4 \mathrm{~N} \mathrm{HCl}$ in $\mathrm{MeOH} / \mathrm{CH}_{2} \mathrm{Cl}_{2}$ to remove the Boc and $t$-butyl groups allowing the preparation of analogues 32-D and 32-L. Due of the high polarity of these products and associated difficulties to their purification, these compounds were obtained in 37 and $28 \%$ yields respectively.
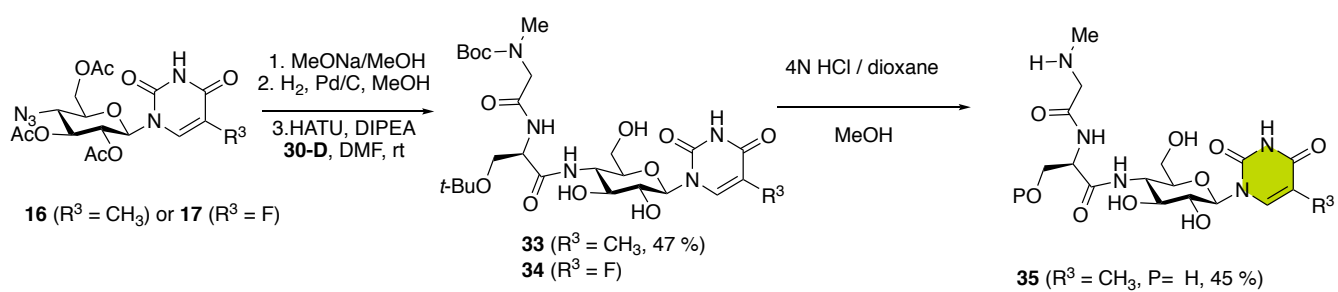

$35\left(\mathrm{R}^{3}=\mathrm{CH}_{3}, \mathrm{P}=\mathrm{H}, 45 \%\right)$

$36\left(R^{3}=F, P=t-B u, 35 \%\right.$ over the for 4 steps $)$

Scheme 4. Preparation of analogues 35-36

To access other compounds, the two uracil derivatives $\mathbf{1 6}$ and $\mathbf{1 7}$, were subjected to a similar reaction sequence, namely Zemplén deacetylation, hydrogenation followed by dipeptide coupling with carboxylic acid 30-D, affording the corresponding peptidonucleosides 33 and $\mathbf{3 4}$ (Scheme 4). Further deprotection with $\mathrm{HCl} 4 \mathrm{~N}$ allowed us to obtain analogues $\mathbf{3 5}$ and 36. For 34, a partial deprotection was obtained leading to $\mathbf{3 6}$ as the major adduct still having the $t$ butyl group on the serine. Attempts to further deprotect led to a very low yield of desired product due to degradation. Taking advantage of the presence of the azide in position C-4 of pyranosyl compound 18, we also prepared compound 40 containing a triazole unit (Scheme 5). To this end we synthesized alkyne 38 from 29-D after its reduction to alcohol 37, oxidation to the aldehyde ${ }^{24}$ and treatment with dimethyl (1-diazo-2-oxopropyl)phosphonate ${ }^{25}$ in the presence of potassium carbonate in $\mathrm{MeOH}$. The $\mathrm{CuAAC}$ reaction ${ }^{26}$ between alkyne $\mathbf{3 8}$ and azide $\mathbf{1 8}$ was carried out in a water/dichloromethane mixture with $\mathrm{CuSO}_{4} \cdot 5 \mathrm{H}_{2} \mathrm{O}$ and ascorbic acid to smoothly give the triazole derivative 39 in $76 \%$ yield. Final removal of the acyl groups with $\mathrm{MeONa}$ in methanol followed by treatment with $4 \mathrm{~N} \mathrm{HCl}$ at room temperature gave, as for $\mathbf{3 6}$, a partial deprotection leading to $\mathbf{4 0}$ having the $t$-butyl group on the serine as the major adduct in $58 \%$ yield. 

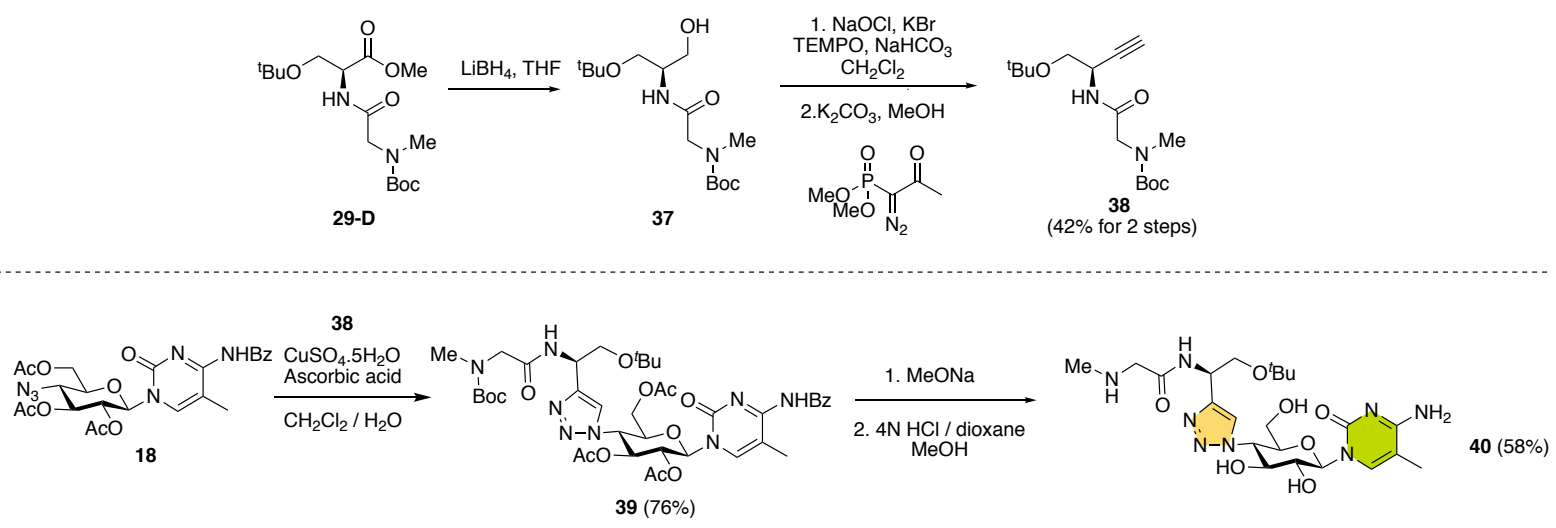

Scheme 5. Preparation of analogue $\mathbf{4 0}$

\section{Conclusion}

In this article, we have successfully prepared a series of peptidonucleosides, analogues of gougerotin. The main modifications relate to the replacement of the natural nucleic base by other pyrimidine bases and the replacement of the carboxamide function at $\mathrm{C} 5$ by an hydroxymethyl group. For the glycosylation stage, a methodological study involving different anomeric leaving groups (acetate, phenylsulfoxide and ortho-hexynylbenzoate) was carried out. Using six different pyrimidines, the sulfoxide donor in combination with TMSOTf as a promoter most generally led to the best yields. The antifungal activities of the synthesized analogues were evaluated in preventive tests against a panel of different pathogens such as Podosphaera fuliginea, Uromyces appendiculatus, Puccinia triticina, Alternaria brassicae Botryotiniacinereal, Zymoseptoria tritici. Although some of the analogs were found to have good bioactivities, none of them showed superior activity to gougerotin itself.

\section{Experimental}

\section{General remarks}

All non-aqueous reactions were run under an inert atmosphere (argon), by using standard techniques for manipulating air-sensitive compounds and the glassware was stored in the oven prior to use. All reagents and solvents were commercially available and were used without further purification. Molecular sieves $4 \AA$ were used as a powder and were activated overnight at $250{ }^{\circ} \mathrm{C}$ and under reduced pressure, in a Kugelrohr apparatus or with a micro-wave for 45 seconds. Reactions were monitored with analytical Merck TLC silica gel 60 F254 plates and visualized under UV $(254 \mathrm{~nm})$ and stained with $\mathrm{KMNO}_{4}$ or vanillin. Column chromatography was done with Merck Geduran silical gel Si $60(40-63 \mu \mathrm{m})$ and Redisep Rf columns (silica gel Si 60, 40-63 $\mu \mathrm{m}$ ) on an Interchim puriFlash ${ }^{\circledR}$ apparatus and on a Teledyne Isco combiflash Rf. Preparative thin-layer chromatography was performed on silica gel $60 \mathrm{~F} 2540.5 \mathrm{~mm}$ $20 \times 20 \mathrm{~cm}$ plates and visualised under UV $(254 \mathrm{~nm})$. Deuterated chloroform used for NMR analyses was generally neutralized by addition of anhydrous and granular $\mathrm{K}_{2} \mathrm{CO}_{3}$. NMR spectra were recorded with AM 300, AVANCE 300 and AVANCE 500 Brüker spectrometers. Chemical shifts are given in parts per million, referenced to the solvent peak of $\mathrm{CDCl}_{3}$, defined at $77.2 \mathrm{ppm}\left({ }^{13} \mathrm{C} \mathrm{NMR}\right)$ and $7.26 \mathrm{ppm}\left({ }^{1} \mathrm{H} \mathrm{NMR}\right)$ or to the solvent peak of $\mathrm{CD}_{3} \mathrm{OD}$, defined at $49.9 \mathrm{ppm}\left({ }^{13} \mathrm{C} \mathrm{NMR}\right)$ and $3.34 \mathrm{ppm}\left({ }^{1} \mathrm{H} \mathrm{NMR}\right)$ or to the solvent peak of $\mathrm{D}_{2} \mathrm{O}$, defined at $4.79 \mathrm{ppm}\left({ }^{1} \mathrm{H} \mathrm{NMR}\right)$ or to the solvent peak of DMSO-d 6 , defined at $39.5 \mathrm{ppm}\left({ }^{13} \mathrm{C} \mathrm{NMR}\right)$ and $2.50 \mathrm{ppm}\left({ }^{1} \mathrm{H} \mathrm{NMR}\right)$. Data are reported as follow: chemical shifts, multiplicity ( $\mathrm{s}=$ singlet, $\mathrm{d}=$ doublet, $\mathrm{t}=$ triplet, $\mathrm{q}=$ quadruplet, $\mathrm{m}=$ multiplet, $\mathrm{bs}=$ broad singlet), coupling constant (in $\mathrm{Hz}$ ) and integration. IR spectra were recorded on a Perkin-Elmer Spectrum BX instrument with an FT-IR system. Optical rotation were measured on an Anton Paar MCP300 polarimeter using a cell of 1-dm-length path. Mass spectra were recorded with Waters Micromass LCT Premier mass spectrometer.

Methyl 2,3-di- $O$-acetyl-4,6-O-benzylidene- $\alpha$-D-galactopyranoside 2a. To a suspension of methyl $\alpha$-Dgalactopyranoside $(5.0 \mathrm{~g}, 25.75 \mathrm{mmol}, 1.0$ eq.) and camphor-10-sulfonic acid (119.6 mg, $0.52 \mathrm{mmol}, 0.02 \mathrm{eq}$.) in dry chloroform $(400 \mathrm{~mL})$ under argon atmosphere was added dropwise benzaldehyde dimethyl acetal $(5.4 \mathrm{~mL}, 36.05$ mmol, 1.4 eq.). The resulting mixture was stirred for $24 \mathrm{~h}$ at $80^{\circ} \mathrm{C}$. Solvent was then removed and the residue was diluted in EtOAc $(75 \mathrm{~mL})$, neutralized with triethylamine then washed with water $(75 \mathrm{~mL})$. Aqueous phase was extracted with EtOAc $(10 \times 50 \mathrm{~mL})$. The organic layers were combined, dried over $\mathrm{Na}_{2} \mathrm{SO}_{4}$, filtered and concentrated under reduced pressure to afford the pure 4,6- $\mathrm{O}$-benzylidene acetal intermediate $(6.52 \mathrm{~g}, 23.12 \mathrm{mmol}, 90 \%)$ as a white 
powder (Experimental data are in agreement with those reported in the literature). ${ }^{27}$ To a stirred solution of the 4,6$O$-benzylidene acetal $(2.47 \mathrm{~g}, 8.76 \mathrm{mmol}, 1 \mathrm{eq}$.$) in pyridine (14 \mathrm{~mL})$ was added acetic anhydride $(6.62 \mathrm{~mL}, 70.1$ mmol, 8 eq.). The resulting mixture was stirred at room temperature for $12 \mathrm{~h}$. Solvent was then removed and the residue was co-evaporated with toluene $(3 \times 20 \mathrm{~mL})$. The crude product was purified by flash chromatography on silica gel (Heptane/EtOAc 70:30 to 60:40) to afford product 2a (3.20 g, $8.74 \mathrm{mmol}$, quantitative) as a white powder. $[\alpha]_{\mathrm{D}}{ }^{25}+202.3\left(c=0.6, \mathrm{CHCl}_{3}\right) ;{ }^{1} \mathrm{H} \mathrm{NMR}\left(300 \mathrm{MHz}, \mathrm{CDCl}_{3}\right) \delta 7.53-7.46\left(\mathrm{~m}, 2 \mathrm{H}, H_{\mathrm{Ar}}\right), 7.41-7.31\left(\mathrm{~m}, 3 \mathrm{H}, H_{\mathrm{Ar}}\right), 5.50$ $(\mathrm{s}, 1 \mathrm{H}, H 7), 5.35\left(\mathrm{dd}, 1 \mathrm{H}, J_{2, l}=3.0 \mathrm{~Hz}, J_{2,3}=10.5 \mathrm{~Hz}, H 2\right), 5.30\left(\mathrm{dd}, 1 \mathrm{H}, J_{3,4}=2.5 \mathrm{~Hz}, J_{3,2}=10.5 \mathrm{~Hz}, H 3\right), 5.07(\mathrm{~d}, 1 \mathrm{H}$, $\left.J_{1,2}=3.0 \mathrm{~Hz}, H 1\right), 4.45\left(\mathrm{~d}, 1 \mathrm{H}, J_{4,3}=2.5 \mathrm{~Hz}, H 4\right), 4.27\left(\mathrm{dd}, 1 \mathrm{H}, J_{6,5}=1.5 \mathrm{~Hz}, J_{6,6}{ }^{\prime}=12.5 \mathrm{~Hz}, H 6\right), 4.05\left(\mathrm{dd}, 1 \mathrm{H}, J_{6}, 5=\right.$ $1.5 \mathrm{~Hz}, J_{6}, \sigma^{=}=12.5 \mathrm{~Hz}, H 6$ '), $3.74(\mathrm{~m}, 1 \mathrm{H}, H 5), 3.40\left(\mathrm{~s}, 3 \mathrm{H}, \mathrm{OCH}_{3}\right), 2.07$ (s, 3H, OCOCH$\left.)_{3}\right), 2.06\left(\mathrm{~s}, 3 \mathrm{H}, \mathrm{OCOCH}_{3}\right)$; ${ }^{13} \mathrm{C}$ NMR $\left(75 \mathrm{MHz}, \mathrm{CDCl}_{3}\right) \delta 170.9(C=\mathrm{O}), 170.4(C=\mathrm{O}), 137.7(C \mathrm{qAr}) 129.2\left(C \mathrm{H}_{\mathrm{Ar}}\right), 128.4\left(C \mathrm{H}_{\mathrm{Ar}}\right), 126.4\left(C \mathrm{H}_{\mathrm{Ar}}\right)$, 101.1 (C7), 98.0 (C1), 74.1 (C4), 69.3 (C6), 68.8 (C3), 68.3 (C2), $62.2(C 5), 55.7\left(\mathrm{OCH}_{3}\right), 21.2\left(\mathrm{OCOCH}_{3}\right), 21.1$ $\left(\mathrm{OCOCH}_{3}\right)$. IR $v\left(\right.$ film, cm $\left.{ }^{-1}\right) 2914(=\mathrm{C}-\mathrm{H}), 2866\left(\mathrm{CH}_{3}\right), 1746(\mathrm{C}=\mathrm{O}) ;$ ESIHRMS m/z=389.1214 [M+Na] $]^{+}$ $\mathrm{C}_{18} \mathrm{H}_{22} \mathrm{O} 8 \mathrm{Na}$ requires 389.1212 .

Methyl 2,3-di- $\boldsymbol{O}$-acetyl-6-O-benzyl- $\boldsymbol{\alpha}$-D-galactopyranoside 3a. A solution of 2a (2.72 g, $7.4 \mathrm{mmol}, 1 \mathrm{eq}$.) and $4 \AA$ molecular sieves $(5 \mathrm{~g})$ in dry $\mathrm{CH}_{2} \mathrm{Cl}_{2}(50 \mathrm{~mL})$ was stirred for $1 \mathrm{~h}$ at room temperature under argon atmosphere. The mixture was cooled to $-78{ }^{\circ} \mathrm{C}$ and $\mathrm{Et}_{3} \mathrm{SiH}(1.18 \mathrm{~mL}, 7.4 \mathrm{mmol}, 1$ eq.) and $\mathrm{TfOH}(330 \mu \mathrm{L}, 3.7 \mathrm{mmol}, 0.5$ eq. $)$ were added successively. After being stirred for $15 \mathrm{~min}$ at $-78{ }^{\circ} \mathrm{C}, \mathrm{Et} 3 \mathrm{SiH}$ (1 eq.) and $\mathrm{TfOH}(0.5 \mathrm{eq})$ were added again. After 15 min on same conditions, new additions of $\mathrm{Et}_{3} \mathrm{SiH}$ ( 0.5 eq.) and $\mathrm{TfOH}$ ( 0.5 eq.) were done. Finally, after again 15 min, a final addition of $\mathrm{TfOH}(0.5$ eq. $)$ was realized. The resulting mixture was stirred at $-78^{\circ} \mathrm{C}$ for 30 min then diluted with $\mathrm{CHCl}_{3}(20 \mathrm{~mL})$ and poured in saturated aqueous solution of sodium bicarbonate $(40 \mathrm{~mL})$. The organic layer was extract with $\mathrm{CHCl}_{3}(3 \times 25 \mathrm{~mL})$, dried over $\mathrm{Na}_{2} \mathrm{SO}_{4}$, filtered and concentrated under vacuum. The crude mixture was directly dissolved in $\mathrm{MeCN}(25 \mathrm{~mL})$ and treated with a diluted aqueous solution of $\mathrm{HBF}_{4}(0.25 \mathrm{M}, 25 \mathrm{~mL})$. The resulting solution was stirred at room temperature for $1 \mathrm{~h} 30$ and then quenched with saturated solution of $\mathrm{NaHCO}_{3}$ until neutralization. Aqueous layer was extracted with EtOAc $(3 \times 15 \mathrm{~mL})$. Organic layers were dried over $\mathrm{Na}_{2} \mathrm{SO}_{4}$, filtered and concentrated under vacuum. The crude product was purified by flash chromatography on silica gel (Heptane/EtOAc 90:10 to 60:40) to afford the clean product 3a $(1.52 \mathrm{~g}, 4.1 \mathrm{mmol}, 63 \%)$ as a colorless oil. $[\alpha]_{\mathrm{D}}{ }^{25}$ $+119.6\left(c=0.9, \mathrm{CHCl}_{3}\right) ;{ }^{1} \mathrm{H} \mathrm{NMR}\left(300 \mathrm{MHz}, \mathrm{CDCl}_{3}\right) \delta 7.36-7.26\left(\mathrm{~m}, 5 \mathrm{H}, H_{\mathrm{Ar}}\right), 5.30\left(\mathrm{dd}, 1 \mathrm{H}, J_{2,1}=3.0 \mathrm{~Hz}, J_{2,3}=\right.$ $10.5 \mathrm{~Hz}, H 2), 5.24\left(\mathrm{dd}, 1 \mathrm{H}, J_{3,4}=2.5 \mathrm{~Hz}, J_{3,2}=10.5 \mathrm{~Hz}, H 3\right), 5.00\left(\mathrm{~d}, 1 \mathrm{H}, J_{1,2}=3.0 \mathrm{~Hz}, H 1\right), 4.60\left(\mathrm{~d}, 1 \mathrm{H}, J_{H, H}=12.0\right.$ $\left.\mathrm{Hz}, \mathrm{CH}_{2} \mathrm{Ph}\right) 4.54\left(\mathrm{~d}, 1 \mathrm{H}, J_{H, H}=12.0 \mathrm{~Hz}, \mathrm{CH}_{2} \mathrm{Ph}\right), 4.24(\mathrm{~m}, 1 \mathrm{H}, H 4), 3.97\left(\mathrm{t}, 1 \mathrm{H}, J_{5,4}=J_{5,6}=4.5 \mathrm{~Hz}, H 5\right), 3.78(\mathrm{dd}, 1 \mathrm{H}$, $\left.J_{6,5}=4.5 \mathrm{~Hz}, J_{6,6}=10.0 \mathrm{~Hz}, H 6\right), 3.73\left(\mathrm{dd}, 1 \mathrm{H}, J_{6,5}=4.5 \mathrm{~Hz}, J_{6,6}=10.0 \mathrm{~Hz}, H 6^{\prime}\right), 3.38\left(\mathrm{~s}, 3 \mathrm{H}, \mathrm{OCH}_{3}\right), 3.01$ (bs, $1 \mathrm{H}$, $\mathrm{OH}), 2.08\left(\mathrm{~s}, 3 \mathrm{H}, \mathrm{OCOCH}_{3}\right), 2.06\left(\mathrm{~s}, 3 \mathrm{H}, \mathrm{OCOCH}_{3}\right) ;{ }^{13} \mathrm{C} \mathrm{NMR}\left(75 \mathrm{MHz}, \mathrm{CDCl}_{3}\right) \delta 170.6(\mathrm{C}=\mathrm{O}), 170.3(\mathrm{C}=\mathrm{O}), 137.5$ $\left(C \mathrm{q}_{\mathrm{Ar}}\right), 128.7\left(C \mathrm{H}_{\mathrm{Ar}}\right), 128.1\left(\mathrm{CH}_{\mathrm{Ar}}\right), 127.9\left(\mathrm{CH}_{\mathrm{Ar}}\right), 97.6(C 1), 74.1\left(C \mathrm{H}_{2} \mathrm{Ph}\right), 70.4(C 2), 70.4(C 6), 69.4(C 4), 68.5$ (C3), 68.1 (C5), $55.6\left(\mathrm{OCH}_{3}\right), 21.2\left(\mathrm{OCOCH}_{3}\right), 21.1\left(\mathrm{OCOCH}_{3}\right) ; \mathrm{IR} v\left(\mathrm{film}, \mathrm{cm}^{-1}\right) 3466(\mathrm{O}-\mathrm{H}), 2934(=\mathrm{C}-\mathrm{H}), 1738$ $(\mathrm{C}=\mathrm{O})$; ESIHRMS $m / z=391.1369[\mathrm{M}+\mathrm{Na}]^{+}$. $\mathrm{C}_{18} \mathrm{H}_{24} \mathrm{O}_{8} \mathrm{Na}$ requires 391.1369.

Methyl 2,3-di- $\boldsymbol{O}$-acetyl-4-azido-6- $\boldsymbol{O}$-benzyl- $\boldsymbol{\alpha}$-D-glucopyranoside 4a. Galactopyranose 3a (77 mg, $0.209 \mathrm{mmol}, 1$ eq.) was co-evaporated 2 times with toluene $(5 \mathrm{~mL})$ and then diluted in dry $\mathrm{CH}_{2} \mathrm{Cl}_{2}(\mathrm{C}=0.1 \mathrm{M}, 2 \mathrm{~mL})$ under argon atmosphere. Pyridine $\left(0.18 \mathrm{~mL}, 2.29 \mathrm{mmol}, 11 \mathrm{eq}\right.$.) was added and the mixture was cooled to $0{ }^{\circ} \mathrm{C}$. Triflic anhydride ( $70 \mu \mathrm{L}, 0.418 \mathrm{mmol}, 2$ eq.) was added and the mixture stirred for $90 \mathrm{~min}$ at $0{ }^{\circ} \mathrm{C}$. The mixture was quenched by addition of $10 \%$ aqueous $\mathrm{NaHCO}_{3}$ solution $(20 \mathrm{~mL})$, the phases were separated and the organic layer was extracted with $3 \%$ hydrochloric acid $(3 \times 10 \mathrm{~mL})$, with water $(10 \mathrm{~mL})$, dried over $\mathrm{Na}_{2} \mathrm{SO}_{4}$ and was concentrated under vacuum. The resulting crude product was co-evaporated with toluene $(3 \mathrm{x})$ to remove all traces of pyridine and dried for $1 \mathrm{~h}$ under vacuum. The yellow residue was dissolved in dry DMF $(2.4 \mathrm{~mL})$, sodium azide (332 $\mathrm{mg}, 5.12 \mathrm{mmol}, 12 \mathrm{eq}$.) was added, and the reaction mixture was stirred overnight. The mixture was diluted with water and EtOAc $(15 \mathrm{~mL})$ and the phases were separated. The organic phase was washed with brine $(3 \times 15 \mathrm{~mL})$, dried over $\mathrm{Na}_{2} \mathrm{SO}_{4}$ and concentrated under vacuum. The residue was purified by flash chromatography on silica gel (Heptane/EtOAc 90:10 to $80: 20)$ to afford $4 \mathbf{a}(69 \mathrm{mg}, 0.175 \mathrm{mmol}, 84 \%)$ as a colorless oil. $[\alpha]_{\mathrm{D}}{ }^{25}+136.2\left(c=0.6, \mathrm{CHCl}_{3}\right) ;{ }^{1} \mathrm{H} \mathrm{NMR}(300$ $\left.\mathrm{MHz}, \mathrm{CDCl}_{3}\right) \delta 7.39-7.26\left(\mathrm{~m}, 5 \mathrm{H}, H_{\mathrm{Ar}}\right), 5.41\left(\mathrm{t}, 1 \mathrm{H}, J_{3,2}=10.0 \mathrm{~Hz}, H 3\right), 4.91\left(\mathrm{~d}, 1 \mathrm{H}, J_{l, 2}=4.0 \mathrm{~Hz}, H 1\right), 4.85(\mathrm{dd}, 1 \mathrm{H}$, $\left.J_{2, I}=4.0 \mathrm{~Hz}, J_{2,3}=10.0 \mathrm{~Hz}, H 2\right), 4.65\left(\mathrm{~d}, 1 \mathrm{H}, J_{H, H}=12.0 \mathrm{~Hz}, \mathrm{CH}_{2} \mathrm{Ph}\right) 4.54\left(\mathrm{~d}, 1 \mathrm{H}, J_{H, H}=12.0 \mathrm{~Hz}, \mathrm{CH} \mathrm{H}_{2} \mathrm{Ph}\right), 3.79(\mathrm{t}, 1 \mathrm{H}$, $\left.J_{4,3}=J_{4,5}=10.0 \mathrm{~Hz}, H 4\right), 3.74-3.41\left(\mathrm{~m}, 3 \mathrm{H}, H 5, H 6, H 6\right.$ '), $3.36\left(\mathrm{~s}, 3 \mathrm{H}, \mathrm{OCH}_{3}\right), 2.08(\mathrm{~s}, 3 \mathrm{H}, \mathrm{OCOCH}), 2.05(\mathrm{~s}, 3 \mathrm{H}$, $\left.\mathrm{OCOCH}_{3}\right) ;{ }^{13} \mathrm{C} \mathrm{NMR}\left(75 \mathrm{MHz}, \mathrm{CDCl}_{3}\right) \delta 170.5(\mathrm{C}=\mathrm{O}), 170.0(\mathrm{C}=\mathrm{O}), 137.8\left(\mathrm{Cq}_{\mathrm{Ar}}\right), 128.7\left(\mathrm{CH}_{\mathrm{Ar}}\right), 128.0\left(\mathrm{CH}_{\mathrm{Ar}}\right), 128.0$ $\left(\mathrm{CH}_{\mathrm{Ar}}\right), 97.3(\mathrm{C} 1), 73.8\left(\mathrm{CH}_{2} \mathrm{Ph}\right), 71.2(\mathrm{C} 2), 70.9(\mathrm{C3}), 69.3(\mathrm{C5}), 68.5(C 6), 60.2(C 4), 55.6\left(\mathrm{O}-\mathrm{CH}_{3}\right), 21.0$ $\left(\mathrm{OCOCH}_{3}\right), 21.0\left(\mathrm{OCOCH}_{3}\right)$; IR $v\left(\right.$ film, $\left.\mathrm{cm}^{-1}\right) 2935\left(\mathrm{CH}_{2}\right), 2106\left(\mathrm{~N}_{3}\right), 1746(\mathrm{C}=\mathrm{O})$; ESIHRMS $m / z=457.1712[\mathrm{M}+$ $\left.\mathrm{CH}_{3} \mathrm{CN}+\mathrm{Na}\right]^{+}$. $\mathrm{C}_{20} \mathrm{H}_{26} \mathrm{~N}_{4} \mathrm{O}_{7} \mathrm{Na}$ requires 457.1699 .

1,2,3,6-Tetra- $\boldsymbol{O}$-acetyl-4-azido- $\boldsymbol{\alpha}$-D-glucopyranose 5 . To a stirred solution of $\mathbf{4 a}(57 \mathrm{mg}, 0.16 \mathrm{mmol}, 1 \mathrm{eq}$.) in acetic anhydride $(0.5 \mathrm{~mL})$ was added dropwise at $0{ }^{\circ} \mathrm{C} \mathrm{H}_{2} \mathrm{SO}_{4}(10 \mu \mathrm{L})$. The resulting mixture was stirred overnight at room 
temperature and then diluted by cold water. After being stirred for $1 \mathrm{~h}$, the phases were separated and the aqueous phase was extracted with EtOAc $(3 \times 2 \mathrm{~mL})$. Organic layers were combined, neutralized with aqueous $\mathrm{NaHCO}_{3}(4$ $\mathrm{mL})$, then washed with brine $(2 \times 3 \mathrm{~mL})$, dried over $\mathrm{Na}_{2} \mathrm{SO}_{4}$ and evaporated under vacuum. The residue was then purified by flash chromatography on silica gel (Heptane/EtOAc 70:30 to 60:40) to afford clean product 5 ( $\alpha / \beta$ 8:2) (43 mg, $0.12 \mathrm{mmol}, 73 \%$ ) as a colorless oil (Experimental data agree with those reported in the literature). ${ }^{28}$

2,3,6-Tri-O-acetyl-4-azido-D-glucopyranose 5-OH. To a stirred solution of 5 ( 1 eq., $50 \mathrm{mg}, 0.13 \mathrm{mmol})$ in THF (1.3 $\mathrm{mL}$ ) was added benzylamine ( 1.5 eq., $22 \mu \mathrm{L}, 0.20 \mathrm{mmol})$ and the resulting mixture was stirred at room temperature for $14 \mathrm{~h}$ under inert atmosphere. After addition of $1 \mathrm{~N} \mathrm{HCl}(0.1 \mathrm{~mL})$, the reaction mixture was stirred for one more hour. The reaction mixture was diluted with $1 \mathrm{~N} \mathrm{HCl}(6 \mathrm{~mL})$ and extracted with $\mathrm{CH}_{2} \mathrm{Cl}_{2}(3 \times 8 \mathrm{~mL})$. The combined extracts were dried over anhydrous $\mathrm{Na}_{2} \mathrm{SO}_{4}$ and concentrated under reduce pressure. The residue was purified by flash chromatography on silica gel (Heptane/EtOAc 70:30 to 40:60) to give the corresponding hemiacetal 5-OH (28 mg, $0.085 \mathrm{mmol}, 63 \%, \alpha / \beta=70: 30)$ as a colorless oil. ${ }^{1} \mathrm{H}$ NMR $\left(300 \mathrm{MHz}, \mathrm{CDCl}_{3}\right) \delta 5.51\left(\mathrm{t}, 0.7 \mathrm{H}, J_{3,2}=J_{3,4}=10.0 \mathrm{~Hz}\right.$, $H 3 \alpha$ ), 5,38 (bs, $0.7 \mathrm{H}, H 1 \alpha), 5.19\left(\mathrm{t}, 0.3 \mathrm{H}, J_{3,2}=J_{3,4}=9.5 \mathrm{~Hz}, H 3 \beta\right), 4.82\left(\mathrm{dd}, 0.7 \mathrm{H}, J_{2,3}=10.0 \mathrm{~Hz}, J_{2,1}=3.5 \mathrm{~Hz}, H 2 \alpha\right)$, $4.80\left(\mathrm{dd}, 0.3 \mathrm{H}, J_{2,3}=9.5 \mathrm{~Hz}, J_{2,1}=8.0 \mathrm{~Hz}, H 2 \beta\right), 4.70\left(\mathrm{t}, 0.3 \mathrm{H}, J_{l, 2}=7.0 \mathrm{~Hz}, H 1 \beta\right), 4.39\left(\mathrm{dd}, 0.3 \mathrm{H}, J_{6,6^{\prime}}=12.0 \mathrm{~Hz}, J_{6,5}\right.$ $=2.5 \mathrm{~Hz}, H 6 \beta), 4.37\left(\mathrm{dd}, 0.7 \mathrm{H}, J_{6,6^{\prime}}=12.0 \mathrm{~Hz}, J_{6,5}=2.5 \mathrm{~Hz}, H 6 \alpha\right), 4.22\left(\mathrm{dd}, 1 \mathrm{H}, J_{6,6}=12.0 \mathrm{~Hz}, J^{\prime}, 5=4.0 \mathrm{~Hz}, H^{\prime} \beta\right.$, $\left.H 6^{\prime} \alpha\right), 4.02\left(\mathrm{ddd}, 0.7 \mathrm{H}, J_{5,4}=10.0 \mathrm{~Hz}, J_{5,6^{\prime}}=4.0 \mathrm{~Hz}, J_{5,6}=2.5 \mathrm{~Hz}, H 5 \alpha\right), 3.63\left(\mathrm{t}, 0.3 \mathrm{H}, J_{4,3}=J_{4,5}=10 \mathrm{~Hz}, H 4 \beta\right), 3.58$ $\left(\mathrm{t}, 0.7 \mathrm{H}, J_{4,3}=J_{4,5}=10.0 \mathrm{~Hz}, H 4 \alpha\right), 3.48\left(\mathrm{ddd}, 0.3 \mathrm{H}, J_{5,4}=10.0 \mathrm{~Hz}, J_{5,6}=4.0 \mathrm{~Hz}, J_{5,6}=2.5 \mathrm{~Hz}, H 5 \beta\right), 2.10(\mathrm{~s}, 3 \mathrm{H}$, $\left.\mathrm{OCOCH}_{3}\right), 2.09\left(\mathrm{~s}, 3 \mathrm{H}, \mathrm{OCOCH}_{3}\right), 2.06\left(\mathrm{~s}, 3 \mathrm{H}, \mathrm{OCOCH}_{3}\right) ;{ }^{13} \mathrm{C} \mathrm{NMR}\left(75 \mathrm{MHz}, \mathrm{CDCl}_{3}\right) \delta 170.9(\mathrm{C}=\mathrm{O}), 170.8(\mathrm{C}=\mathrm{O})$, $170.7(C=\mathrm{O}), 170.4(C=\mathrm{O}), 169.9(C=\mathrm{O}), 95.5(C 1 \beta), 90.6(C 1 \alpha), 73.5(C 2 \beta), 73.2(C 3 \beta), 72.5(C 5 \beta), 71.5(C 2 \alpha)$, $70.6(\mathrm{C} 3 \alpha), 67.8(C 5 \alpha), 63.0(C 6 \beta), 62.8(C 6 \alpha), 60.4(C 4 \beta), 60.3(C 4 \alpha), 21.0\left(\mathrm{COCH}_{3}\right) 20.9\left(\mathrm{COCH}_{3}\right), 20.8\left(\mathrm{COCH}_{3}\right)$; IR $v\left(\right.$ film, cm $\left.{ }^{-1}\right) 3458(\mathrm{O}-\mathrm{H}), 2960\left(\mathrm{CH}_{3}\right), 2108\left(\mathrm{~N}_{3}\right), 1739(\mathrm{C}=\mathrm{O}) ;$ ESIHRMS $\mathrm{m} / z=354.0903[\mathrm{M}+\mathrm{Na}]^{+}$. $\mathrm{C}_{12} \mathrm{H}_{17} \mathrm{~N}_{3} \mathrm{O}_{8} \mathrm{Na}$ requires 354.0913 .

1-O-(ortho-Hexynylbenzoyl)-2,3,4-tri- $O$-acetyl-4-azido-D-glucopyranose 6. To a stirred solution of 5-OH (1 eq., $28 \mathrm{mg}, 0.08 \mathrm{mmol}$ ) and ortho-(hex-1-yn-1-yl)benzoic acid (1.2 eq., $20 \mathrm{mg}, 0.10 \mathrm{mmol})$, in $\mathrm{CH}_{2} \mathrm{Cl}_{2}(0.3 \mathrm{~mL})$ were added DCC (1.5 eq., $26 \mathrm{mg}, 0.13 \mathrm{mmol})$ and DMAP (1.5 eq., $15 \mathrm{mg}, 0.13 \mathrm{mmol})$ under inert atmosphere. After being stirred for $3 \mathrm{~h}$ at room temperature, the reaction mixture was diluted with $\mathrm{CH}_{2} \mathrm{Cl}_{2}(1 \mathrm{~mL})$ and washed with saturated aqueous $\mathrm{NaHCO}_{3}(1 \mathrm{~mL})$ and brine $(1 \mathrm{~mL})$. The organic layer was dried over anhydrous $\mathrm{Na}_{2} \mathrm{SO}_{4}$ and then concentrated under reduced pressure. The residue was purified by flash chromatography on silica gel (Heptane/EtOAc 90:10 to $70: 30)$ to provide $6(36 \mathrm{mg}, 84 \% ; \alpha / \beta=3: 6)$ as a white solid. ${ }^{1} \mathrm{H}$ NMR $\left(300 \mathrm{MHz}, \mathrm{CDCl}_{3}\right) \delta 7.93-7.79\left(\mathrm{~m}, 1 \mathrm{H}, H_{\mathrm{Ar} \alpha}\right.$, $\left.H_{\mathrm{Ar}} \beta\right), 7.53-7.21\left(\mathrm{~m}, 3 \mathrm{H}, H_{\mathrm{Ar}} \alpha, H_{\mathrm{Ar}} \beta\right), 6.51\left(\mathrm{~d}, 0.4 \mathrm{H}, J_{l, 2}=3.5 \mathrm{~Hz}, H 1 \alpha\right), 5.86\left(\mathrm{~d}, 0.6 \mathrm{H}, J_{1,2}=8.0 \mathrm{~Hz}, H 1 \beta\right), 5.55(\mathrm{t}$, $\left.0.4 \mathrm{H}, J_{3,2}=J_{3,4}=10.0 \mathrm{~Hz}, H 3 \alpha\right), 5.23\left(\mathrm{t}, 0.6 \mathrm{H}, J_{3,2}=J_{3,4}=9.0 \mathrm{~Hz}, H 3 \beta\right), 5.16\left(\mathrm{dd}, 0.6 \mathrm{H}, J_{2,1}=8.0 \mathrm{~Hz}, J_{2,3}=9.0 \mathrm{~Hz}\right.$,

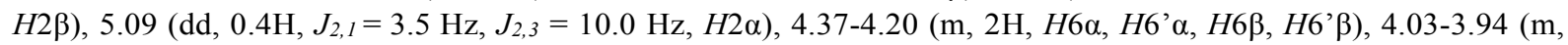
$0.4 \mathrm{H}, H 5 \alpha), 3.72\left(\mathrm{t}, 0.6 \mathrm{H}, J_{4,3}=J_{4,5}=9.0 \mathrm{~Hz}, H 4 \beta\right), 3.69\left(\mathrm{t}, 0.4 \mathrm{H}, J_{4,3}=J_{4,5}=10.0 \mathrm{~Hz}, H 4 \alpha\right), 3.65-3.58(\mathrm{~m}, 0.6 \mathrm{H}$, $H 5 \beta), 2.50-2.36(\mathrm{~m}, 2 \mathrm{H}, H 7), 2.07\left(\mathrm{~s}, 3 \mathrm{H}, \mathrm{COCH}_{3}\right), 2.04\left(\mathrm{~s}, 3 \mathrm{H}, \mathrm{COCH}_{3}\right), 1.93\left(\mathrm{~s}, 1.2 \mathrm{H}, \mathrm{COCH} \mathrm{C}_{3}\right), 1.92(\mathrm{~s}, 1.8 \mathrm{H}$, $\left.\mathrm{COCH}_{3} \beta\right), 1.64-1.49(\mathrm{~m}, 2 \mathrm{H}, H 8), 1.49-1.34(\mathrm{~m}, 2 \mathrm{H}, H 9), 0.88\left(\mathrm{t}, 3 \mathrm{H}, J_{\mathrm{CH}, 9}=7.0 \mathrm{~Hz}, \mathrm{CH}\right) ;{ }^{13} \mathrm{C} \mathrm{NMR}(75 \mathrm{MHz}$, $\left.\mathrm{CDCl}_{3}\right) \delta 170.7(C=\mathrm{O}), 170.2(C=\mathrm{O}), 170.0(C=\mathrm{O}), 169.9(C=\mathrm{O}), 164.2(C \mathrm{q}), 163.3(C \mathrm{q}), 135.4\left(C \mathrm{H}_{\mathrm{Ar}}\right), 135.0\left(C \mathrm{H}_{\mathrm{Ar}}\right)$, $132.8\left(C_{\mathrm{Ar}}\right), 132.7\left(\mathrm{CH}_{\mathrm{Ar}}\right), 130.9\left(C \mathrm{H}_{\mathrm{Ar}}\right), 129.8(\mathrm{Cq} \mathrm{Ar}), 129.2\left(C \mathrm{q}_{\mathrm{Ar}}\right), 127.6\left(C \mathrm{H}_{\mathrm{Ar}}\right), 127.4\left(C \mathrm{H}_{\mathrm{Ar}}\right), 126.1\left(C \mathrm{q}_{\mathrm{Ar}}\right)$,

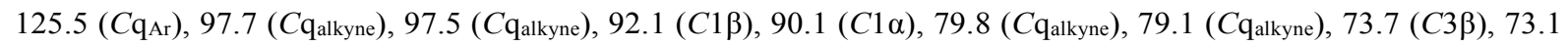
(C5 $)$ ), $70.8(C 3 \alpha), 70.6(C 2 \beta, C 5 \alpha), 69.7(C 2 \alpha), 62.7(C 6 \beta), 62.5(C 6 \alpha), 60.2(C 4 \beta), 60.0(C 4 \alpha), 30.9(C 8 \beta), 30.8$ $(C 8 \alpha), 22.3(C 9 \beta, C 9 \alpha), 20.9\left(\mathrm{COCH}_{3}\right), 20.9\left(\mathrm{COCH}_{3}\right), 20.8\left(\mathrm{COCH}_{3}\right), 20.7\left(\mathrm{COCH}_{3}\right), 20.7\left(\mathrm{COCH}_{3}\right), 19.8(C 7 \beta)$, $19.7(C 7 \alpha), 13.9\left(\mathrm{CH}_{3} \beta\right), 13.8\left(\mathrm{CH}_{3} \alpha\right)$; IR $v$ (film, $\left.\mathrm{cm}^{-1}\right) 2959\left(\mathrm{CH}_{3}\right), 2935\left(\mathrm{CH}_{2}\right), 2229$ (alkyne), $2110\left(\mathrm{~N}_{3}\right), 1745$ $(\mathrm{C}=\mathrm{O})$; ESIHRMS $m / z=538.1804[\mathrm{M}+\mathrm{Na}]^{+} . \mathrm{C}_{25} \mathrm{H}_{29} \mathrm{~N}_{3} \mathrm{O}_{9} \mathrm{Na}$ requires 538.1801.

Phenyl 2,3-di- $\boldsymbol{O}$-acetyl-4,6- $\boldsymbol{O}$-benzylidene-thio- $\boldsymbol{\beta}$-D-galactopyranoside $\mathbf{2 b}$. To a stirred solution of $\mathrm{Na}(52 \mathrm{mg}, 20$ $\mathrm{mol} \%$ ) in dry $\mathrm{MeOH}(113 \mathrm{~mL})$ was added phenyl 2,3,4,6-tetra- $O$-acetyl-thio- $\beta$-D-galactoside (5 g, $11.35 \mathrm{mmol}, 1$ eq.) under argon atmosphere. The resulting mixture was stirred at room temperature for $2 \mathrm{~h}$ and then neutralized with Dowex ${ }^{\circledR} \mathrm{H}^{+}$, filtered on celite ${ }^{\circledR}$, concentrated under reduced pressure and co-evaporated with toluene to afford the deprotected adduct 1b. This latter (3.03 g, $11.3 \mathrm{mmol}, 1$ eq.) was then dissolved in dry $\mathrm{MeCN}(24 \mathrm{~mL})$ then benzaldehyde dimethyl acetal $(2.7 \mathrm{~mL}, 17.8 \mathrm{mmol}, 1.6$ eq.) and p-TsOH (15 mol\%, $300 \mathrm{mg})$ were added. The mixture was stirred at room temperature for $2 \mathrm{~h}$ under argon atmosphere and then neutralized with Et $3 \mathrm{~N}(2 \mathrm{~mL})$. Water $(30$ $\mathrm{mL})$ and EtOAc $(50 \mathrm{~mL})$ were added and the aqueous layer was extracted with EtOAc $(2 \times 50 \mathrm{~mL})$. Organic layers were dried over $\mathrm{Na}_{2} \mathrm{SO}_{4}$, filtered and concentrated under reduced pressure. The crude product was recrystallized with heptane/EtOAc 70:30 to afford the desired compound $(3.3 \mathrm{~g}, 82 \%)$ as a white solid (experimental data agree with those reported in the literature). ${ }^{29}$ The obtained product ( $3.27 \mathrm{~g}, 11.1 \mathrm{mmol}, 1 \mathrm{eq}$.) was dissolved in pyridine $(12 \mathrm{~mL})$. $\mathrm{Ac}_{2} \mathrm{O}(6 \mathrm{~mL})$ was added and the mixture was stirred at room temperature overnight. Pyridine was co-evaporated with 
toluene. The crude product was recrystallized with Heptane/EtOAc 55/45 to afford $\mathbf{2 b}(3.61 \mathrm{~g}, 73 \%)$ as a white solid (experimental data agree with those reported in the literature). Erreur ! Signet non défini.

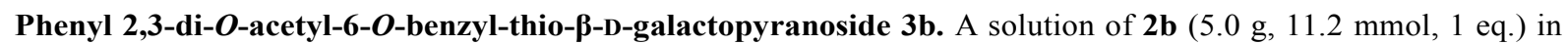
dry $\mathrm{MeCN}(110 \mathrm{~mL})$ was cooled to $0{ }^{\circ} \mathrm{C}$ and $\mathrm{Et}_{3} \mathrm{SiH}(10.8 \mathrm{~mL}, 67.4 \mathrm{mmol}, 1$ eq. $)$ was added, followed by the addition of $\mathrm{Cu}(\mathrm{OTf})_{2}\left(200 \mathrm{mg}, 0.56 \mathrm{mmol}, 0.05\right.$ eq.). The resulting mixture was stirred at $0{ }^{\circ} \mathrm{C}$ for $1 \mathrm{~h}$ and then hydrolysed with a saturated solution of $\mathrm{NaHCO}_{3}(60 \mathrm{~mL})$. The phases were separated and organic layer was dried over $\mathrm{Na}_{2} \mathrm{SO}_{4}$, filtered and concentrated under vacuum. The desired product was obtained in mixture with the silylated one (6.28 g, ratio 30:70). The crude mixture was directly dissolved in $\mathrm{MeCN}(20 \mathrm{~mL})$ and treated with a diluted aqueous solution of $\mathrm{HBF}_{4}(\mathrm{C}=0.25 \mathrm{M}, 20 \mathrm{~mL})$. The resulting solution was stirred at room temperature for $1.5 \mathrm{~h}$ and then quenched with saturated solution of $\mathrm{NaHCO}_{3}$ until neutralization. Aqueous layer was extracted with EtOAc $(3 \times 15 \mathrm{~mL})$. Organic layers were dried over $\mathrm{Na}_{2} \mathrm{SO}_{4}$, filtered and concentrated under vacuum. The crude product was purified by flash chromatography (Heptane/EtOAc 90:10 to 40:60) to afford product $\mathbf{3 b}$ ( $4.43 \mathrm{~g}, 9.93 \mathrm{mmol}, 88 \%)$ as a colorless oil. (Experimental data agree with those reported in the literature). ${ }^{30}$

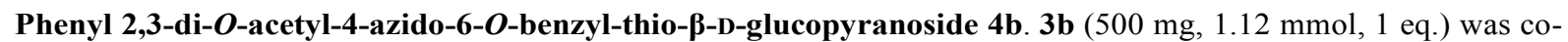
evaporated 2 times with toluene $(5 \mathrm{~mL})$ and then diluted in dry $\mathrm{CH}_{2} \mathrm{Cl}_{2}(\mathrm{C}=0.1 \mathrm{M}, 11.2 \mathrm{~mL})$ under argon atmosphere. Pyridine (1.0 mL, $12.32 \mathrm{mmol}, 11$ eq.) was added and the mixture was cooled to $0{ }^{\circ} \mathrm{C}$. Triflic anhydride $(280 \mu \mathrm{L}, 1.68$ mmol, 2 eq.) was added and the mixture stirred for $90 \mathrm{~min}$ at $0{ }^{\circ} \mathrm{C}$. The mixture was quenched by addition of $10 \%$ aqueous $\mathrm{NaHCO}_{3}$ solution $(50 \mathrm{~mL})$, the phases were separated and the organic layer was extracted with $3 \%$ hydrochloric acid $(3 \times 20 \mathrm{~mL})$, with water $(20 \mathrm{~mL})$, dried over $\mathrm{Na}_{2} \mathrm{SO}_{4}$ and was concentrated under vacuum. The resulting crude product was co-evaporated with toluene $(3 \mathrm{x})$ to remove all traces of pyridine and dried for $1 \mathrm{~h}$ under vacuum. The yellow residue was dissolved in dry DMF $(10 \mathrm{~mL})$, sodium azide $(1.6 \mathrm{~g}, 25.0 \mathrm{mmol}, 12 \mathrm{eq}$.) was added, and the reaction mixture was stirred overnight. The mixture was diluted with water and EtOAc $(20 \mathrm{~mL})$ and the phases were separated. The organic phase was washed with brine $(3 \times 30 \mathrm{~mL})$, dried over $\mathrm{Na}_{2} \mathrm{SO}_{4}$ and concentrated under vacuum. The residue was purified by flash chromatography on silica gel (Heptane/EtOAc 90:10 to 80:20) to afford product $4 \mathrm{~b}$ (347 mg, $0.736 \mathrm{mmol}, 66 \%)$ as a colorless oil. $[\alpha]_{\mathrm{D}}{ }^{25}-4.4\left(c=1.1, \mathrm{CHCl}_{3}\right) ;{ }^{1} \mathrm{H} \mathrm{NMR}\left(300 \mathrm{MHz}, \mathrm{CDCl}_{3}\right)$ $\delta$ 7.42-7.34 (m, 2H, $\left.H_{\mathrm{Ar}}\right), 7.30-7.09\left(\mathrm{~m}, 8 \mathrm{H}, H_{\mathrm{Ar}}\right), 5.04\left(\mathrm{dd}, 1 \mathrm{H}, J_{3,2}=9.5 \mathrm{~Hz}\right.$ and $\left.J_{3,4}=10.0 \mathrm{~Hz}, H 3\right), 4.79(\mathrm{dd}, 1 \mathrm{H}$, $J_{2,1}=10.0 \mathrm{~Hz}$ and $\left.J_{2,3}=9.5 \mathrm{~Hz}, H 2\right), 4.56\left(\mathrm{~d}, 1 \mathrm{H}, J_{1,2}=10.0 \mathrm{~Hz}, H 1\right), 4.52\left(\mathrm{~d}, 1 \mathrm{H}, J_{H, H}=12.0 \mathrm{~Hz}, \mathrm{CH} 2 \mathrm{Ph}\right), 4.45(\mathrm{~d}$, $\left.1 \mathrm{H}, J_{H, H}=12.0 \mathrm{~Hz}, \mathrm{CH} H_{2} \mathrm{Ph}\right), 3.69\left(\mathrm{dd}, 1 \mathrm{H}, J_{6,6^{\prime}}=11.0 \mathrm{~Hz}\right.$ and $\left.J_{6,5}=2.0 \mathrm{~Hz}, H 6\right), 3.64\left(\mathrm{t}, 1 \mathrm{H}, J_{4,3}=J_{4,5}=10.0 \mathrm{~Hz}, H 4\right)$ $3.62\left(\mathrm{dd}, 1 \mathrm{H}, J_{6^{\prime}, 6}=11.0 \mathrm{~Hz}, J_{6^{\prime}, 5}=4.0 \mathrm{~Hz}, H 6^{\prime}\right), 3.31\left(\mathrm{ddd}, 1 \mathrm{H}, J_{5,4}=10.0 \mathrm{~Hz}, J_{5,6^{\prime}}=4.0 \mathrm{~Hz}\right.$ and $\left.J_{5,6}=2.0 \mathrm{~Hz}, H 5\right)$, $1.96\left(\mathrm{~s}, 6 \mathrm{H}, \mathrm{CH}_{3}\right) ;{ }^{13} \mathrm{C} \mathrm{NMR}\left(75 \mathrm{MHz}, \mathrm{CDCl}_{3}\right) \delta 170.2(\mathrm{C}=\mathrm{O}), 169.7(\mathrm{C}=\mathrm{O}), 138.0\left(\mathrm{Cq}_{\mathrm{Ar}}\right), 133.3\left(\mathrm{CH}_{\mathrm{Ar}}\right), 132.0\left(C \mathrm{q}_{\mathrm{Ar}}\right)$, $129.2\left(\mathrm{CH}_{\mathrm{Ar}}\right), 128.6\left(\mathrm{CH}_{\mathrm{Ar}}\right), 128.5\left(\mathrm{CH}_{\mathrm{Ar}}\right), 128.0(\mathrm{CHC} \mathrm{Ar}), 127.9\left(\mathrm{CH}_{A r}\right), 85.9(\mathrm{C} 1), 78.4(C 5) 75.0(C 3), 73.8$ $\left(\mathrm{CH}_{2} \mathrm{Ph}\right), 70.4(C 2), 69.0(C 6), 60.0(C 4), 21.0\left(\mathrm{COCH}_{3}\right), 20.9\left(\mathrm{COCH}_{3}\right)$; IR v (film, $\left.\mathrm{cm}^{-1}\right) 3122(=\mathrm{C}-\mathrm{H}), 2926\left(-\mathrm{CH}_{2}\right)$, $2110\left(\mathrm{~N}_{3}\right), 1752(\mathrm{C}=\mathrm{O})$; ESIHRMS $m / z=494.1360[\mathrm{M}+\mathrm{Na}]^{+} . \mathrm{C}_{23} \mathrm{H}_{25} \mathrm{~N}_{3} \mathrm{O}_{6} \mathrm{SNa}$ requires 494.1362.

Phenyl 2,3,6-tri- $\boldsymbol{O}$-acetyl-4-azido-thio-p-D-glucopyranoside. To a stirred solution of $\mathbf{4 b}$ (1.48g, $3.14 \mathrm{mmol}, 1$ eq.) in dry acetic anhydride $(5.6 \mathrm{~mL})$ at $0{ }^{\circ} \mathrm{C}$ was added dropwise a solution of $\mathrm{NaI}(471 \mathrm{mg}, 3.14 \mathrm{mmol}, 1 \mathrm{eq})$ in $\mathrm{MeCN}$ $(1.9 \mathrm{~mL})$ followed by $\mathrm{BF}_{3} \cdot \mathrm{OEt}_{2}(1.16 \mathrm{~mL}, 4.71 \mathrm{mmol}, 1.5 \mathrm{eq})$. After completion of the reaction, the reaction mixture was quenched with aqueous $\mathrm{Na}_{2} \mathrm{~S}_{2} \mathrm{O}_{3}$ until neutralization and extracted with EtOAc $(15 \mathrm{~mL})$. The organic layer was washed with water $(3 \times 15 \mathrm{~mL})$, brine $(3 \times 15 \mathrm{~mL})$, dried over $\mathrm{Na}_{2} \mathrm{SO}_{4}$ and concentrated under vacuum. The crude product was purified by flash chromatography (Heptane/EtOAc 90:10 to 75:25) to afford the desired clean product $(0.8242 \mathrm{~g}, 62 \%)$ as a colorless oil. $[\alpha]_{\mathrm{D}}{ }^{25}-2.80\left(c=1, \mathrm{CHCl}_{3}\right) ;{ }^{1} \mathrm{H} \mathrm{NMR}\left(300 \mathrm{MHz}, \mathrm{CDCl}_{3}\right): 7.56-7.44\left(\mathrm{~m}, 2 \mathrm{H}, H_{\mathrm{Ar}}\right)$, 7.37-7.28 (m, $\left.3 \mathrm{H}, H_{\mathrm{Ar}}\right), 5.21\left(\mathrm{t}, 1 \mathrm{H}, J_{2,3}=J_{3,4}=10.5 \mathrm{~Hz}, H_{3}\right), 4.91\left(\mathrm{t}, 1 \mathrm{H}, \mathrm{J}_{1,2}=\mathrm{J}_{2,3}=10.5 \mathrm{~Hz}, H_{2}\right), 4.68\left(\mathrm{~d}, 1 \mathrm{H}, J_{1,2}=10.5\right.$ $\left.\mathrm{Hz}, H_{1}\right), 4.52-4.45\left(\mathrm{dd}, 1 \mathrm{H}, J_{6,6}=12.5 \mathrm{~Hz}\right.$ and $\left.J_{6,5}=2.0 \mathrm{~Hz}, H_{6}\right), 4.30-4.21\left(\mathrm{dd}, 1 \mathrm{H}, J_{6,6}=12.5 \mathrm{~Hz}\right.$ and $J_{6}, 5=5.0 \mathrm{~Hz}$, $\left.\mathrm{H}_{6}{ }^{\prime}\right) 3.64\left(\mathrm{t}, 1 \mathrm{H}, J_{4,5}=J_{3,4}=10.0 \mathrm{~Hz}, H_{4}\right), 3.54-3.46\left(\mathrm{ddd}, 1 \mathrm{H}, J_{4,5}=10.2 \mathrm{~Hz}, J_{6}, 5=5.0 \mathrm{~Hz}\right.$ and $\left.\mathrm{J}_{6,5}=2.0 \mathrm{~Hz}, H_{5}\right), 2.13(\mathrm{~s}$, $\left.3 \mathrm{H}, \mathrm{CH}_{3}\right), 2.12(\mathrm{~s}, 3 \mathrm{H}, \mathrm{CH}), 2.11(\mathrm{~s}, 3 \mathrm{H}, \mathrm{CH}) ;{ }^{13} \mathrm{C} \mathrm{NMR}\left(75 \mathrm{MHz}, \mathrm{CDCl}_{3}\right): 170.38(\mathrm{C}=\mathrm{O}), 169.87(\mathrm{C}=\mathrm{O}), 169.48$ $(C=\mathrm{O}), 133.4\left(2 * C \mathrm{H}_{\mathrm{Ar}}\right), 128.9\left(2 * C \mathrm{H}_{\mathrm{Ar}}\right), 128.5\left(C \mathrm{q}_{\mathrm{Ar}}\right), 85.6(C 1), 75.9(C 5), 74.7(C 3), 69.8(C 2), 62.8(C 6), 59.9$ $(C 4), 20.8\left(\mathrm{COCH}_{3}\right), 20.6\left(\mathrm{COCH}_{3}\right)$; IR $v\left(\right.$ film, $\left.\mathrm{cm}^{-1}\right): 2952(-\mathrm{CH} 2), 2109(\mathrm{~N} 3), 1745(\mathrm{C}=\mathrm{O})$; ESIHRMS m/z = $446.1004[\mathrm{M}+\mathrm{Na}]^{+}$. $\mathrm{C}_{18} \mathrm{H}_{21} \mathrm{~N}_{3} \mathrm{O}_{7} \mathrm{SNa}$ requires 446.0998.

2,3,6-Tri-O-acetyl-4-azido-ß-D-glucopyranosyl phenyl sulfoxide 7. To a stirred solution the previous described sulfide compound ( $1.4 \mathrm{~g}, 3.27 \mathrm{mmol}, 1$ eq.) in $\mathrm{CH}_{2} \mathrm{Cl}_{2}(31 \mathrm{~mL})$ was added $m$-CPBA $(75 \%, 0.85 \mathrm{~g}, 3.92 \mathrm{mmol}, 1.2$ eq.) at $-78^{\circ} \mathrm{C}$ under argon atmosphere. The resulting mixture was stirred at $-30{ }^{\circ} \mathrm{C}$ overnight and a solution of aqueous $\mathrm{Na}_{2} \mathrm{~S}_{2} \mathrm{O}_{3} / \mathrm{NaHCO}_{3}$ 50:50 $(20 / 20 \mathrm{~mL})$ was then added. The solution was allowed to warm at room temperature, extracted with EtOAc $(2 \times 30 \mathrm{~mL})$. Organics layers were dried over $\mathrm{Na}_{2} \mathrm{SO}_{4}$, filtered and concentrated under vacuum. The crude product was purified by flash chromatography (Heptane/EtOAc 80:20 to 50:50) to afford the clean product 7 as a mixture of two diastereoisomers $(\mathrm{dr}=1: 1,1.2 \mathrm{~g}, 84 \%)$ as a colorless oil. ${ }^{1} \mathrm{H} \mathrm{NMR}\left(300 \mathrm{MHz}, \mathrm{CDCl}_{3}\right): 7.71$ - 
$7.50\left(\mathrm{~m}, 10 \mathrm{H}, H_{\mathrm{Ar}}\right), 5.40-5.14\left(\mathrm{~m}, 4 \mathrm{H}, H_{2}, H_{3}\right), 4.51-4.44\left(\mathrm{~m}, 2 \mathrm{H}, H_{6}\right), 4.31\left(\mathrm{dd}, 1 \mathrm{H}, J_{6,6}=12.0 \mathrm{~Hz}\right.$ and $\left.J_{6,5}=1.5 \mathrm{~Hz}, H_{6}\right)$, $4.24\left(\mathrm{~d}, 1 \mathrm{H}, J_{2,1}=9.5 \mathrm{~Hz}, \mathrm{H}_{1}\right), 4.22-4.09\left(\mathrm{~m}, 2 \mathrm{H}, H_{1}, H_{6}\right), 3.70-3.56\left(\mathrm{~m}, 2 \mathrm{H}, H_{4}\right), 3.52-3.47\left(\mathrm{~m}, 1 \mathrm{H}, H_{5}\right), 3.43-3.33(\mathrm{~m}$, $\left.1 \mathrm{H}, \mathrm{H}_{5}\right), 2.14\left(\mathrm{~s}, 3 \mathrm{H}, \mathrm{CH}_{3}\right), 2.12\left(\mathrm{~s}, 3 \mathrm{H}, \mathrm{CH}_{3}\right), 2.11\left(\mathrm{~s}, 3 \mathrm{H}, \mathrm{CH}_{3}\right), 2.08\left(\mathrm{~s}, 3 \mathrm{H}, \mathrm{CH}_{3}\right), 2.02\left(\mathrm{~s}, 3 \mathrm{H}, \mathrm{CH}_{3}\right), 1.86(\mathrm{~s}, 3 \mathrm{H}$, $\left.\mathrm{CH}_{3}\right) ;{ }^{13} \mathrm{C}$ NMR $\left(75 \mathrm{MHz}, \mathrm{CDCl}_{3}\right): 170.2(\mathrm{C}=\mathrm{O}), 170.1(\mathrm{C}=\mathrm{O}), 169.9(\mathrm{C}=\mathrm{O}), 169.5(\mathrm{C}=\mathrm{O}), 169.2(C=\mathrm{O}), 131.7\left(C_{\mathrm{Ar}}\right)$, $131.5\left(C_{\mathrm{Ar}}\right), 129.0\left(C_{\mathrm{Ar}}\right), 128.9\left(C_{\mathrm{Ar}}\right), 125.7\left(C_{\mathrm{Ar}}\right), 125.4\left(C_{\mathrm{Ar}}\right), 92.4(C 1), 90.1(C 1), 77.5(C 5), 77.0(C 5), 74.9(C 3)$, 74.6 (C3), 67.5 (C2), $67.1(C 2), 62.4$ (C6), 62.2 (C6), $59.6(C 4), 59.3(C 4), 20.7\left(\mathrm{COCH}_{3}\right), 20.6\left(\mathrm{COCH}_{3}\right), 20.5$ $\left(\mathrm{COCH}_{3}\right) ; \mathrm{IR} \vee\left(\right.$ film, $\left.\mathrm{cm}^{-1}\right): 2942(-\mathrm{CH} 2), 2110(\mathrm{~N} 3), 1741(\mathrm{C}=\mathrm{O}) ;$ ESIHRMS m/z $=901.2006[2 \mathrm{M}+\mathrm{Na}]^{+}$. $\mathrm{C}_{36} \mathrm{H}_{42} \mathrm{~N}_{6} \mathrm{O}_{16} \mathrm{~S}_{2} \mathrm{Na}$ requires 901.2006 .

2,3-Di- $\boldsymbol{O}$-acetyl-4-azido-6- $\boldsymbol{O}$-benzyl- $\beta$-D-glucopyranosyl phenyl sulfoxide 8 . To a stirred solution of $\mathbf{4 b}$ (1.0 g, 2.12 mmol, 1 eq.) in $\mathrm{CH}_{2} \mathrm{Cl}_{2}\left(21 \mathrm{~mL}\right.$ ) was added $m$-CPBA (above $75 \%, 550 \mathrm{mg}, 3.18 \mathrm{mmol}, 1.5 \mathrm{eq}$.) at $-78{ }^{\circ} \mathrm{C}$ under argon atmosphere. The resulting mixture was stirred at $-30{ }^{\circ} \mathrm{C}$ overnight and dimethylsulfide $(0.2 \mathrm{~mL})$ was then added. The solution was allowed to warm at room temperature, diluted with $\mathrm{CH}_{2} \mathrm{Cl}_{2}(10 \mathrm{~mL})$, washed with water $(8 \mathrm{~mL})$, with a saturated aqueous solution of $\mathrm{NaHCO}_{3}(8 \mathrm{~mL})$, with water again $(8 \mathrm{~mL})$ and finally with brine $(8 \mathrm{~mL})$. The organic phase was dried with $\mathrm{Na}_{2} \mathrm{SO}_{4}$, filtered and concentrated under vacuum. The crude product was purified by flash chromatography on silica gel (Heptane/EtOAc 80:20 to 60:40) to afford product 8 (914 $\mathrm{mg}, 1.875 \mathrm{mmol}, 89 \%)$ as a colorless oil and as a mixture of two diastereoisomers $(\mathrm{dr}=2: 3) .{ }^{1} \mathrm{H} \mathrm{NMR}\left(300 \mathrm{MHz}, \mathrm{CDCl}_{3}\right) \delta 7.71-7.59(\mathrm{~m}, 2 \mathrm{H}$, $\left.H_{\mathrm{Ar}}\right), 7.55-7.42\left(\mathrm{~m}, 3 \mathrm{H}, H_{\mathrm{Ar}}\right), 7.42-7.21\left(\mathrm{~m}, 5 \mathrm{H}, H_{\mathrm{Ar}}\right), 5.29\left(\mathrm{t}, 0.4 \mathrm{H}, J_{2 x, 1 x}=J_{2 x, 3 x}=9.5 \mathrm{~Hz}, H 2 \mathrm{x}\right), 5.24\left(\mathrm{t}, 0.6 \mathrm{H}, J_{2 y, 1 y}\right.$ $\left.=J_{2 y, 3 y}=9.0 \mathrm{~Hz} H 2 \mathrm{y}\right), 5.19\left(\mathrm{t}, 0.4 \mathrm{H}, J_{3 x, 2 x}=J_{3 x, 4 x}=9.5 \mathrm{~Hz}, H 3 \mathrm{x}\right), 5.16\left(\mathrm{t}, 0.6 \mathrm{H}, J_{3 y, 2 y}=J_{3 y, 4 y}=9.0 \mathrm{~Hz}, H 3 \mathrm{y}\right), 4.56-$ $4.36\left(\mathrm{~m}, 2.6 \mathrm{H}, \mathrm{CH}_{2} \mathrm{Ph}, H 1 \mathrm{y}\right), 4.25\left(\mathrm{~d}, 0.4 \mathrm{H}, J_{1 x, 2 x}=9.5 \mathrm{~Hz}, H 1 \mathrm{x}\right), 3.82-3.69\left(\mathrm{~m}, 2.2 \mathrm{H}, H 6, H 6^{\prime}\right), 3.69-3.56(\mathrm{~m}, 1 \mathrm{H}$, $H 4$ ), 3.48-3.38 (dt, $0.6 \mathrm{H}, J b_{5 y, 4 y}=10.0 \mathrm{~Hz}, J_{5 y, 6^{\prime} y}=5.0 \mathrm{~Hz}$ and $\left.J_{5 y, 6 y}=2.5 \mathrm{~Hz}, H 5 \mathrm{y}\right), 3.36-3.24$ (ddd, $0.4 \mathrm{H}, J b_{5 x, 4 x}=$ $10.0 \mathrm{~Hz}, J_{5 x, 6^{\prime} x}=6.0 \mathrm{~Hz}$ and $\left.J_{5 x, 6 x}=4.0 \mathrm{~Hz}, H 5 \mathrm{x}\right), 2.10\left(\mathrm{~s}, 1.2 \mathrm{H}, \mathrm{OCOCH}_{3} \mathrm{x}\right), 2.08\left(\mathrm{~s}, 1.8 \mathrm{H}, \mathrm{OCOCH}_{3 \mathrm{y}}\right), 2.06(\mathrm{~s}, 1.2 \mathrm{H}$, $\left.\mathrm{OCOCH}_{3} \mathrm{x}\right), 1.83\left(\mathrm{~s}, 1.8 \mathrm{H}, \mathrm{OCOCH}_{3} \mathrm{y}\right) ;{ }^{13} \mathrm{C} \mathrm{NMR}\left(75 \mathrm{MHz}, \mathrm{CDCl}_{3}\right) \delta 170.4(C=\mathrm{O}), 170.3(C=\mathrm{O}), 169.7(C=\mathrm{O}), 169.4$ $(C=\mathrm{O}), 139.6\left(C \mathrm{q}_{\mathrm{Ar}}\right), 139.0\left(C \mathrm{q}_{\mathrm{Ar}}\right), 137.8\left(C \mathrm{q}_{\mathrm{Ar}}\right), 137.7\left(C \mathrm{q}_{\mathrm{Ar}}\right), 131.8\left(C \mathrm{H}_{\mathrm{Ar}}\right), 131.7\left(C \mathrm{H}_{\mathrm{Ar}}\right), 129.2\left(C \mathrm{H}_{\mathrm{Ar}}\right), 129.1$ $\left(C \mathrm{H}_{\mathrm{Ar}}\right), 128.6\left(\mathrm{CH}_{\mathrm{Ar}}\right), 128.5\left(\mathrm{CH}_{\mathrm{Ar}}\right), 128.1\left(C \mathrm{H}_{\mathrm{Ar}}\right), 128.0\left(C \mathrm{H}_{\mathrm{Ar}}\right), 127.9\left(C \mathrm{H}_{\mathrm{Ar}}\right), 127.9\left(C \mathrm{H}_{\mathrm{Ar}}\right), 125.8\left(C \mathrm{H}_{\mathrm{Ar}}\right), 125.6$ $\left(\mathrm{CH}_{\mathrm{Ar}}\right), 93.0(C 1 \mathrm{y}), 90.5(C 1 \mathrm{x}), 79.3(C 5 \mathrm{x}), 79.0(C 5 \mathrm{y}), 75.1(C 2 \mathrm{y}), 74.8(C 3 \mathrm{y}), 73.8\left(\mathrm{CH}_{2} \mathrm{Ph}\right), 68.6(C 6 \mathrm{x}), 68.4(C 6 \mathrm{y})$, $67.8(C 2 x), 67.4(C 3 x), 59.4(C 4 y), 59.2(C 4 x), 20.9\left(\mathrm{COCH}_{3}\right), 20.8\left(\mathrm{COCH}_{3}\right), 20.7\left(\mathrm{COCH}_{3}\right) ; \mathrm{IR} v\left(\right.$ film, $\left.\mathrm{cm}^{-1}\right) 2988$ $(=\mathrm{C}-\mathrm{H}), 2901\left(-\mathrm{CH}_{2}\right), 2110\left(\mathrm{~N}_{3}\right), 1755(\mathrm{C}=\mathrm{O})$; ESIHRMS $m / z=510.1312[\mathrm{M}+\mathrm{Na}]^{+} . \mathrm{C}_{23} \mathrm{H}_{25} \mathrm{~N}_{3} \mathrm{O}_{7} \mathrm{SNa}$ requires 510.1311 .

$\mathrm{N}$-Acetyl-cytosine 9. To a stirred solution of cytosine (500 mg, $4.5 \mathrm{mmol}, 1$ eq.) in pyridine $(2.5 \mathrm{~mL})$ was added acetic anhydride (2.1 mL, $22.05 \mathrm{mmol}, 5$ eq.). The resulting mixture was stirred overnight at room temperature then diluted with EtOAc $(2.0 \mathrm{~mL})$ and stirred again for $30 \mathrm{~min}$ at room temperature. The resulting white solid was filtered, washed with EtOAc, co-evaporated with toluene and dried under vacuum to afford clean product $9(0.6521 \mathrm{~g}, 95 \%)$ as a white solid (experimental data agree with those reported in the literature). ${ }^{31}$

$\mathrm{N}$-Benzoyl-5-methyl-cytosine 10. To a suspension of 5-methyl-cytosine (1.5 g, $12 \mathrm{mmol}$, 1eq.) in dry $\mathrm{MeCN}$ (40 $\mathrm{mL}$ ) was added benzoic anhydride ( $3.25 \mathrm{~g}, 14.4 \mathrm{mmol}, 1.2$ eq.) followed by DMAP (293 mg, $2.4 \mathrm{mmol}, 0.2$ eq.) under argon atmosphere. The resulting mixture was refluxed for $24 \mathrm{~h}$ then $\mathrm{EtOH}(25 \mathrm{~mL})$ was added to the hot solution. The solution was cooled to room temperature and the resulting solid was filtered, washed with $\mathrm{EtOH}(15 \mathrm{~mL})$ and $\mathrm{Et}_{2} \mathrm{O}$ $(15 \mathrm{~mL})$ and dried under vacuum to afford clean product $\mathbf{1 0}(1.913 \mathrm{~g}, 70 \%)$ as a white solid (experimental data agree with those reported in the literature). ${ }^{32}$

$\boldsymbol{N}$-Benzoyl-5-fluoro-cytosine 13. To a suspension of 5-fluoro-cytosine (1.5 g, $11.6 \mathrm{mmol}, 1$ eq.) in dry $\mathrm{MeCN}$ (15 $\mathrm{mL}$ ) was added benzoic anhydride $(3.15 \mathrm{~g}, 13.9 \mathrm{mmol}, 1.2$ eq.) followed by DMAP (283 $\mathrm{mg}, 2.32 \mathrm{mmol}, 0.2 \mathrm{eq}$.) under argon atmosphere. The resulting mixture was refluxed for $24 \mathrm{~h}$ then EtOH $(2 \mathrm{~mL})$ was added to the hot solution. The solution was cooled to room temperature and the resulting solid was filtered, washed with EtOH (15 mL) and $\mathrm{Et}_{2} \mathrm{O}(15 \mathrm{~mL})$ and dried under vacuum to afford clean product $13(1.9 \mathrm{~g}, 70 \%)$ as a white solid (experimental data agree with those reported in the literature). ${ }^{33}$

General Procedure for the glycosylation of sulfoxide donors. To a stirred solution of the nitrogen base (1.6 eq.) in dry $\mathrm{MeCN}\left(2 / 3 \mathrm{~V}_{\text {tot }}\right)$ under argon atmosphere was added BSA (4 eq.). The resulting mixture was heated at $60{ }^{\circ} \mathrm{C}$ for $1 \mathrm{~h}$ and then cooled to room temperature. The donor (1 eq.) is stirred with $4 \AA$ molecular sieves in dry MeCN $\left(1 / 3 \mathrm{~V}_{\text {tot }}\right)$ under argon for $1 \mathrm{~h}$. The solution of the nitrogen base was added to the donor and then TMSOTf (1.5 eq.). The resulting mixture was heated at $55{ }^{\circ} \mathrm{C}$ overnight and then quenched with aqueous $\mathrm{NaHCO}_{3}$. The reaction mixture was filtered and the aqueous phase was extracted with EtOAc (5x). The organic layers were combined, washed with $\mathrm{NaCl}$, dried over $\mathrm{Na}_{2} \mathrm{SO}_{4}$, filtered and concentrated under vacuum. The residue was purified by flash chromatography on silica gel to afford the clean product. 
2,3,6-Tri- $\boldsymbol{O}$-acetyl-4-azido-1- $\boldsymbol{N}$-( $\boldsymbol{N}$-acetyl-cytosine)-p-D-glucopyranoside $\mathbf{1 5}$. The General Procedure was followed using 7 (300 mg, $0.68 \mathrm{mmol}), 9$ (184 mg, $1.09 \mathrm{mmol})$, BSA (0.67 mL, $2.72 \mathrm{mmol})$, TMSOTf (0.18 mL, $1.02 \mathrm{mmol})$, $4 \AA$ molecular sieves $(200 \mathrm{mg})$ in dry $\mathrm{MeCN}(13.6 \mathrm{~mL})$. The residue was purified by flash chromatography on silica gel (EtOAc) to afford product $15(304 \mathrm{mg}, 0.65 \mathrm{mmol}, 95 \%)$ as a colorless oil. $[\alpha]_{\mathrm{D}}{ }^{25}+56.6\left(c=1.0, \mathrm{CHCl}_{3}\right) ;{ }^{1} \mathrm{H}$ NMR $\left(300 \mathrm{MHz}, \mathrm{CD}_{3} \mathrm{OD}\right) \delta 8.14\left(\mathrm{~d}, 1 \mathrm{H}, J_{H A r, H A r}=7.5 \mathrm{~Hz}, H_{\mathrm{Ar}}\right), 7.49\left(\mathrm{~d}, 1 \mathrm{H}, J_{H A r, H A r}=7.5 \mathrm{~Hz}, H_{\mathrm{Ar}}\right), 6.12\left(\mathrm{~d}, 1 \mathrm{H}, J_{I, 2}\right.$ $=9.0 \mathrm{~Hz}, H 1), 5.54\left(\mathrm{t}, 1 \mathrm{H}, J_{3,2}=J_{3,4}=9.5 \mathrm{~Hz}, H 3\right), 5.32\left(\mathrm{t}, 1 \mathrm{H}, J_{2,3}=J_{2,1}=9.5 \mathrm{~Hz}, H 2\right), 4.47\left(\mathrm{dd}, 1 \mathrm{H}, J_{6,6^{\prime}}=13.0 \mathrm{~Hz}\right.$ and $\left.J_{6,5}=1.0 \mathrm{~Hz}, H 6\right), 4.31\left(\mathrm{dd}, 1 \mathrm{H}, J_{6,6}=13.0 \mathrm{~Hz}\right.$ and $\left.J_{6,5}=4.5 \mathrm{~Hz}, H 6^{\prime}\right), 4.00-3.93(\mathrm{~m}, 2 \mathrm{H}, H 4, H 5), 2.21(\mathrm{~s}, 3 \mathrm{H}$, $\left.\mathrm{NHCOCH}_{3}\right), 2.14\left(\mathrm{~s}, 3 \mathrm{H}, \mathrm{OCOCH}_{3}\right), 2.12\left(\mathrm{~s}, 3 \mathrm{H}, \mathrm{OCOCH}_{3}\right), 1.94\left(\mathrm{~s}, 3 \mathrm{H}, \mathrm{OCOCH}_{3}\right) ;{ }^{13} \mathrm{C} \mathrm{NMR}\left(75 \mathrm{MHz}, \mathrm{CD}_{3} \mathrm{OD}\right) \delta$ $173.9(C=\mathrm{O}), 173.1(C=\mathrm{O}), 172.0(C=\mathrm{O}), 172.0(C=\mathrm{O}), 165.5\left(C \mathrm{qAr}_{\mathrm{Ar}}\right), 158.6(\mathrm{CqAr}), 147.5\left(\mathrm{CH}_{\mathrm{Ar}}\right), 99.8\left(\mathrm{CH}_{\mathrm{Ar}}\right), 83.6$ (C1), 77.1 (C4), $75.4(C 3), 73.2(C 2), 64.8(C 6), 61.9(C 5), 25.4\left(\mathrm{COCH}_{3}\right), 21.4\left(\mathrm{COCH}_{3}\right), 21.1\left(\mathrm{COCH}_{3}\right)$; IR v (film, $\left.\mathrm{cm}^{-1}\right) 3239(=\mathrm{C}-\mathrm{H}), 2112\left(\mathrm{~N}_{3}\right), 1747(\mathrm{C}=\mathrm{O}), 1663(\mathrm{NH}-\mathrm{C}=\mathrm{O})$; ESIHRMS m/z= 467.1486 [M+H] $]^{+} \mathrm{C}_{18} \mathrm{H}_{23} \mathrm{~N}_{6} \mathrm{O}_{9}$ requires 467.1527 .

2,3,6-Tri- $\boldsymbol{O}$-acetyl-4-azido-1- $\boldsymbol{N}$-thymine- $\boldsymbol{\beta}$-D-glucopyranoside 16. The general procedure was followed using 7 (70 $\mathrm{mg}, 0.16 \mathrm{mmol}), 10$ (32 mg, $0.256 \mathrm{mmol})$, BSA (0.16 mL, $0.64 \mathrm{mmol})$, TMSOTf (43 $\mu \mathrm{L}, 0.24 \mathrm{mmol}, 1.5 \mathrm{eq}.), 4 \AA$ molecular sieves $(150 \mathrm{mg})$ in dry $\mathrm{MeCN}(3 \mathrm{~mL})$. The residue was purified by preparative TLC (Hept/EtOAc 20:80) to afford product $16(63 \mathrm{mg}, 89 \%)$ as a white powder. $[\alpha]_{\mathrm{D}}{ }^{25}+8.7\left(c=0.9, \mathrm{CHCl}_{3}\right) ;{ }^{1} \mathrm{H} \mathrm{NMR}\left(300 \mathrm{MHz}, \mathrm{CDCl}_{3}\right) \delta$ $8.68(\mathrm{~s}, 1 \mathrm{H}, \mathrm{NH}), 7.05\left(\mathrm{~d}, 1 \mathrm{H}, J_{H A r, C H 3}=1.3 \mathrm{~Hz}, H_{\mathrm{Ar}}\right), 5.82\left(\mathrm{~d}, 1 \mathrm{H}, J_{l, 2}=9.5 \mathrm{~Hz}, H 1\right), 5.35\left(\mathrm{t}, 1 \mathrm{H}, J_{3,2}=J_{3,4}=9.5 \mathrm{~Hz}\right.$, $H 3), 5.10\left(\mathrm{t}, 1 \mathrm{H}, J_{2,1}=J_{2,3}=9.5 \mathrm{~Hz}, H 2\right), 4.37\left(\mathrm{dd}, 1 \mathrm{H}, J_{6,6}=12.5 \mathrm{~Hz}\right.$ and $\left.J_{6,5}=1.5 \mathrm{~Hz}, H 6\right), 4.25\left(\mathrm{dd}, 1 \mathrm{H}, J_{6}, 6=12.5\right.$ $\left.\mathrm{Hz}, J_{6,5}=4.5 \mathrm{~Hz}, H 6^{\prime}\right), 3.71-3.64(\mathrm{~m}, 2 \mathrm{H}, H 4, H 5), 2.11\left(\mathrm{~s}, 3 \mathrm{H}, \mathrm{C}(\mathrm{O}) \mathrm{C} H_{3}\right), 2.10\left(\mathrm{~s}, 3 \mathrm{H}, \mathrm{C}(\mathrm{O}) \mathrm{C} H_{3}\right), 1.97(\mathrm{~s}, 3 \mathrm{H}$, $\left.\mathrm{C}(\mathrm{O}) \mathrm{CH}_{3}\right), 1.92\left(\mathrm{~d}, 3 \mathrm{H}, J_{\mathrm{CH} 3, \mathrm{HAr}}=1.0 \mathrm{~Hz}, \mathrm{CArCH}_{3}\right) ;{ }^{13} \mathrm{C} \mathrm{NMR}\left(75 \mathrm{MHz}, \mathrm{CDCl}_{3}\right) \delta 170.4(\mathrm{C}=\mathrm{O}), 169.7(\mathrm{C}=\mathrm{O}), 169.4$ $(C=\mathrm{O}), 162.9\left(C \mathrm{q}_{\mathrm{Ar}}\right), 150.2\left(C \mathrm{q}_{\mathrm{Ar}}\right), 134.3\left(C \mathrm{H}_{\mathrm{Ar}}\right), 112.3\left(C \mathrm{q}_{\mathrm{Ar}}, 80.2(C 1), 75.1(C 5), 73.5(C 3), 69.3(C 2), 62.6(C 6)\right.$, $59.9(C 4), 20.8\left(\mathrm{C}(\mathrm{O}) \mathrm{CH}_{3}\right), 20.6\left(\mathrm{C}(\mathrm{O}) \mathrm{CH}_{3}\right), 20.4\left(\mathrm{C}(\mathrm{O}) \mathrm{CH}_{3}\right), 12.6\left(\mathrm{CArCH}_{3}\right) ; \mathrm{IR} v\left(\mathrm{film}, \mathrm{cm}^{-1}\right) 3220(\mathrm{~N}-\mathrm{H}), 3075$ $(=\mathrm{C}-\mathrm{H}), 2931\left(\mathrm{CH}_{3}\right), 2111\left(\mathrm{~N}_{3}\right), 1748(\mathrm{C}=\mathrm{O}), 1690(\mathrm{NH}-\mathrm{C}=\mathrm{O})$; ESIHRMS $m / z=440.1418\left[\mathrm{M}+\mathrm{H}^{+} . \mathrm{C}_{17} \mathrm{H}_{22} \mathrm{~N}_{5} \mathrm{O}_{9}\right.$ requires 440.1409 .

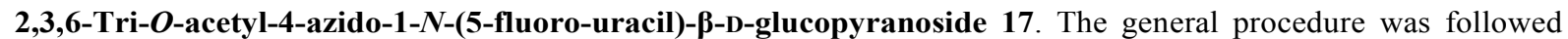
using 7 (70 mg, $0.16 \mathrm{mmol}), 11(33 \mathrm{mg}, 0.256 \mathrm{mmol})$, BSA (0.16 mL, $0.64 \mathrm{mmol})$, TMSOTf (43 $\mu \mathrm{L}, 0.24 \mathrm{mmol}, 1.5$ eq.), $4 \AA$ molecular sieves $(150 \mathrm{mg})$ in dry $\mathrm{MeCN}(3 \mathrm{~mL})$. The residue was purified by preparative TLC (Hept/EtOAc $30: 70)$ to afford product $17(49 \mathrm{mg}, 68 \%)$ as a yellow powder. $[\alpha]_{\mathrm{D}}{ }^{25}+23.0\left(c=1.0, \mathrm{CHCl}_{3}\right) ;{ }^{1} \mathrm{H} \mathrm{NMR}(300 \mathrm{MHz}$, $\left.\mathrm{CDCl}_{3}\right) \delta 9.48\left(\mathrm{~d}, 1 \mathrm{H}, J_{N H, F}=4.5 \mathrm{~Hz}, \mathrm{NH}\right), 7.34\left(\mathrm{~d}, 1 \mathrm{H}, J_{H A r, F}=5.5 \mathrm{~Hz}, H_{\mathrm{Ar}}\right), 5.84\left(\mathrm{~d}, 1 \mathrm{H}, J_{1,2}=9.5 \mathrm{~Hz}, H 1\right), 5.39(\mathrm{t}$, $\left.1 \mathrm{H}, J_{3,2}=J_{3,4}=9.5 \mathrm{~Hz}, H 3\right), 5.03\left(\mathrm{t}, 1 \mathrm{H}, J_{2,3}=J_{2,1}=9.5 \mathrm{~Hz}, H 2\right), 4.39\left(\mathrm{dd}, 1 \mathrm{H}, J_{6,6}=12.5 \mathrm{~Hz}\right.$ and $\left.J_{6,5}=1.5 \mathrm{~Hz}, H 6\right)$, $4.25\left(\mathrm{dd}, 1 \mathrm{H}, J_{6^{\prime}, 6}=12.5 \mathrm{~Hz}\right.$ and $\left.J_{6^{\prime}, 5}=4.5 \mathrm{~Hz}, H 6^{\prime}\right), 3.80-3.71(\mathrm{~m}, 1 \mathrm{H}, H 5), 3.66\left(\mathrm{dd}, 1 \mathrm{H}, J_{4,5}=10.5 \mathrm{~Hz}\right.$ and $J_{4,3}$ $=9.5 \mathrm{~Hz}, \mathrm{H} 4), 2.11(\mathrm{~s}, 3 \mathrm{H}, \mathrm{CH}), 2.10(\mathrm{~s}, 3 \mathrm{H}, \mathrm{CH}), 1.98\left(\mathrm{~s}, 3 \mathrm{H}, \mathrm{CH}_{3}\right) ;{ }^{13} \mathrm{C} \mathrm{NMR}\left(75 \mathrm{MHz}, \mathrm{CDCl}_{3}\right) \delta 170.4(C=\mathrm{O})$, $169.9(C=\mathrm{O}), 169.5(C=\mathrm{O}), 156.4\left(\mathrm{~d}, J_{\mathrm{C}-\mathrm{F}}=27 \mathrm{~Hz}, C \mathrm{q}_{\mathrm{Ar}}\right), 142.4\left(C \mathrm{q}_{\mathrm{Ar}}\right), 139.3\left(C \mathrm{q}_{\mathrm{Ar}}\right), 123.3\left(\mathrm{~d}, J_{\mathrm{C}-\mathrm{F}}=34 \mathrm{~Hz}, C \mathrm{H}_{\mathrm{Ar}}\right)$, 80.5 (C1), 75.0 (C5), $73.1(C 3), 69.5(C 2), 62.4(C 6), 59.7(C 4), 20.8\left(\mathrm{C}(\mathrm{O}) C \mathrm{H}_{3}\right), 20.6\left(\mathrm{C}(\mathrm{O}) C \mathrm{H}_{3}\right), 20.4\left(\mathrm{C}(\mathrm{O}) C \mathrm{H}_{3}\right)$; IR $v\left(\right.$ film, $\left.\mathrm{cm}^{-1}\right) 3222(\mathrm{~N}-\mathrm{H}), 3096(=\mathrm{C}-\mathrm{H}), 2116\left(\mathrm{~N}_{3}\right), 1712(\mathrm{C}=\mathrm{O}), 1673(\mathrm{NH}-\mathrm{C}=\mathrm{O}) ;$ ESIHRMS $m / z=444.1167$ $[\mathrm{M}+\mathrm{H}]^{+} . \mathrm{C}_{16} \mathrm{H}_{19} \mathrm{~N}_{5} \mathrm{O}_{9} \mathrm{~F}$ requires 444.1180 .

2,3,6-Tri- $O$-acetyl-4-azido-1- $N$-(4- $N$-benzoyl-5-methyl-cytosine)- $\beta$-D-glucopyranoside 18 . The general procedure was followed using 7 (70 mg, $0.16 \mathrm{mmol}), 12$ (59 mg, $0.256 \mathrm{mmol})$, BSA (0.16 mL, $0.64 \mathrm{mmol})$, TMSOTf (43 $\mu \mathrm{L}$, $0.24 \mathrm{mmol}, 1.5$ eq.), $4 \AA$ molecular sieves $(150 \mathrm{mg})$ in dry $\mathrm{MeCN}(3 \mathrm{~mL})$. The residue was purified by preparative TLC (Hept/EtOAc 30:70) to afford product $18(76 \mathrm{mg}, 88 \%)$ as a white powder. $[\alpha]_{\mathrm{D}}{ }^{25}+4.1\left(c=1.2, \mathrm{CHCl}_{3}\right) ;{ }^{1} \mathrm{H}$ NMR $\left(300 \mathrm{MHz}, \mathrm{CDCl}_{3}\right) \delta 8.39-8.19\left(\mathrm{~m}, 2 \mathrm{H}, H_{\mathrm{Ar}}\right), 7.59-7.36\left(\mathrm{~m}, 3 \mathrm{H}, H_{\mathrm{Ar}}\right), 7.20\left(\mathrm{~d}, 1 \mathrm{H}, J_{H A r, C H 3}=1.5 \mathrm{~Hz}, H_{\mathrm{Ar}}\right), 5.85$ $\left(\mathrm{d}, 1 \mathrm{H}, J_{1,2}=9.5 \mathrm{~Hz}, H 1\right), 5.35(\mathrm{~m}, 1 \mathrm{H}, H 3), 5.12\left(\mathrm{t}, 1 \mathrm{H}, J_{2,1}=J_{2,3}=9.5 \mathrm{~Hz}, H 2\right), 4.38\left(\mathrm{~d}, 1 \mathrm{H}, J_{6,6^{\prime}}=12.5 \mathrm{~Hz}, H 6\right)$, $4.27\left(\mathrm{dd}, 1 \mathrm{H}, J_{6,6}=12.5 \mathrm{~Hz}\right.$ and $\left.J_{6,5}=3.5 \mathrm{~Hz}, H 6^{\prime}\right), 3.79-3,61(\mathrm{~m}, 2 \mathrm{H}, H 4, H 5), 2.12(\mathrm{~s}, 3 \mathrm{H}, \mathrm{CH}), 2.11(\mathrm{~s}, 3 \mathrm{H}$, $\left.\mathrm{COCH}_{3}\right), 1.98\left(\mathrm{~s}, 3 \mathrm{H}, \mathrm{COCH}_{3}\right) ;{ }^{13} \mathrm{C} \mathrm{NMR}\left(75 \mathrm{MHz}, \mathrm{CDCl}_{3}\right) \delta 180.0(\mathrm{C}=\mathrm{O}), 170.5(\mathrm{C}=\mathrm{O}), 170.0(\mathrm{C}=\mathrm{O}), 169.6(C=\mathrm{O})$, $159.0(C \mathrm{qAr}), 148.2(C \mathrm{qAr}), 136.9(\mathrm{CqAr}), 135.5\left(C \mathrm{H}_{\mathrm{Ar}}\right), 133.0\left(C \mathrm{H}_{\mathrm{Ar}}\right), 130.2\left(C \mathrm{H}_{\mathrm{Ar}}\right), 128.4\left(C \mathrm{H}_{\mathrm{Ar}}\right), 113.3\left(C \mathrm{q}_{\mathrm{Ar}}\right), 80.6$ (C1), 75.4 (C5), $73.8(C 3), 69.8(C 2), 62.9(C 6), 60.1(C 4), 20.8\left(\mathrm{C}(\mathrm{O}) C_{3}\right), 20.6\left(\mathrm{C}(\mathrm{O}) C_{3}\right), 14.0\left(C \mathrm{H}_{3}\right)$; IR v (film, $\left.\mathrm{cm}^{-1}\right) 3072(=\mathrm{C}-\mathrm{H}), 2959(\mathrm{C}-\mathrm{H}), 2110\left(\mathrm{~N}_{3}\right), 1740(\mathrm{C}=\mathrm{O}), 1707(\mathrm{C}=\mathrm{O}), 1656(\mathrm{NH}-\mathrm{C}=\mathrm{O})$; ESIHRMS $m / z=543.1835$ $[\mathrm{M}+\mathrm{H}]^{+} . \mathrm{C}_{24} \mathrm{H}_{27} \mathrm{~N}_{6} \mathrm{O} 9$ requires 543.1840 .

2,3,6-Tri- $\boldsymbol{O}$-acetyl-4-azido-1- $\boldsymbol{N}$-(4- $\boldsymbol{N}$-benzoyl-5-fluoro-cytosine)- $\boldsymbol{\beta}$-D-glucopyranoside $\mathbf{1 9}$. The general procedure was followed using 7 (70 mg, $0.16 \mathrm{mmol}), 13$ (56 mg, $0.256 \mathrm{mmol})$, BSA (0.16 mL, $0.64 \mathrm{mmol})$, TMSOTf (43 $\mu \mathrm{L}$, $0.24 \mathrm{mmol}, 1.5$ eq.), $4 \AA$ molecular sieves $(150 \mathrm{mg})$ in dry $\mathrm{MeCN}(3 \mathrm{~mL})$. The residue was purified by preparative TLC (Hept/EtOAc 20:80) to afford product $19(75 \mathrm{mg}, 75 \%)$ as a white powder. $[\alpha]_{\mathrm{D}}{ }^{20}=+37.4(\mathrm{c}=0.5, \mathrm{MeOH}) ;{ }^{1} \mathrm{H}$ NMR (300 MHz, $\left.\mathrm{CDCl}_{3}\right) \delta 8.26\left(\mathrm{~d}, 2 \mathrm{H}, J_{H A r, H A r}=7.5 \mathrm{~Hz}, H_{\mathrm{Ar}}\right), 7.55\left(\mathrm{t}, 1 \mathrm{H}, J_{H A r, H A r}=7.5 \mathrm{~Hz} H_{\mathrm{Ar}}\right), 7.47-7.39(\mathrm{~m}, 3 \mathrm{H}$, 
$\left.H_{\mathrm{Ar}}\right), 5.82\left(\mathrm{~d}, 1 \mathrm{H}, J_{1,2}=9.5 \mathrm{~Hz}, H 1\right), 5.37\left(\mathrm{t}, 1 \mathrm{H}, J_{3,2}=J_{3,4}=9.5 \mathrm{~Hz}, H 3\right), 5.03\left(\mathrm{t}, 1 \mathrm{H}, J_{2,3}=J_{2, I}=9.5 \mathrm{~Hz}, H 2\right), 4.39(\mathrm{~d}$, $\left.1 \mathrm{H}, J_{H 6, H 6^{\prime}}=12.5 \mathrm{~Hz}, H 6\right), 4.27\left(\mathrm{dd}, 1 \mathrm{H}, J_{H 6^{\prime}, H 6}=12.5 \mathrm{~Hz}\right.$ and $\left.J_{H 6^{\prime}, H 5}=4.0 \mathrm{~Hz}, \mathrm{H} 6^{\prime}\right), 3.72-3.63(\mathrm{~m}, 2 \mathrm{H}, H 4, H 5), 2.12$ (s, 3H, $\left.\mathrm{CH}_{3}\right), 2.11\left(\mathrm{~s}, 3 \mathrm{H}, \mathrm{CH}_{3}\right), 1.99\left(\mathrm{~s}, 3 \mathrm{H}, \mathrm{CH}_{3}\right) ;{ }^{13} \mathrm{C} \mathrm{NMR}\left(75 \mathrm{MHz}, \mathrm{CDCl}_{3}\right) \delta 170.5(\mathrm{C}=\mathrm{O}), 170.1(\mathrm{C}=\mathrm{O}), 169.5$ $(C=\mathrm{O}), 151.8\left(\mathrm{~d}, J_{\mathrm{C}-\mathrm{F}}=19 \mathrm{~Hz}, C \mathrm{qAr}_{\mathrm{Ar}}\right), 146.8\left(C \mathrm{q}_{\mathrm{Ar}}\right), 141.8\left(C \mathrm{q}_{\mathrm{Ar}}\right), 138.7\left(C \mathrm{H}_{\mathrm{Ar}}\right), 135.7\left(C \mathrm{H}_{\mathrm{Ar}}\right), 133.7\left(C \mathrm{H}_{\mathrm{Ar}}\right), 130.4$ $\left(C \mathrm{H}_{\mathrm{Ar}}\right), 128.6\left(C \mathrm{H}_{\mathrm{Ar}}\right), 124.0\left(\mathrm{~d}, J_{\mathrm{C}-\mathrm{F}}=35 \mathrm{~Hz}, C \mathrm{H}_{\mathrm{Ar}}\right), 81.1(C 1), 75.5(C 5), 73.4(C 3), 69.8(C 2), 62.7(C 6), 60.0(C 4)$, $21.0\left(\mathrm{C}(\mathrm{O}) \mathrm{CH}_{3}\right), 20.7\left(\mathrm{C}(\mathrm{O}) \mathrm{CH}_{3}\right), 20.6\left(\mathrm{C}(\mathrm{O}) \mathrm{CH}_{3}\right)$; IR $v\left(\right.$ film, $\left.\mathrm{cm}^{-1}\right) 3100(=\mathrm{C}-\mathrm{H}), 2113\left(\mathrm{~N}_{3}\right), 1754(\mathrm{C}=\mathrm{O}), 1674$ $(\mathrm{NH}-\mathrm{C}=\mathrm{O})$ ESIHRMS $m / z=547.1589[\mathrm{M}+\mathrm{H}]^{+}$. $\mathrm{C}_{23} \mathrm{H}_{24} \mathrm{~N}_{6} \mathrm{O} 9 \mathrm{~F}$ requires 547.1589 .

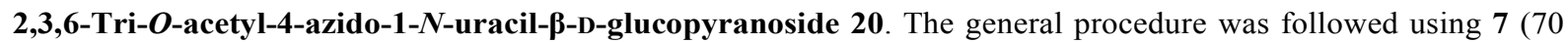
$\mathrm{mg}, 0.16 \mathrm{mmol}), 14(29 \mathrm{mg}, 0.256 \mathrm{mmol})$, BSA $(0.16 \mathrm{~mL}, 0.64 \mathrm{mmol})$, TMSOTf (43 $\mu \mathrm{L}, 0.24 \mathrm{mmol}, 1.5 \mathrm{eq}), 4 \AA$ molecular sieves $(150 \mathrm{mg})$ in dry MeCN $(3 \mathrm{~mL})$. The residue was purified by preparative TLC (Hept/EtOAc 20:80) to afford product $20(63 \mathrm{mg}, 94 \%)$ as a white powder. $[\alpha]_{\mathrm{D}}{ }^{25}+24.1\left(c=1.0, \mathrm{CHCl}_{3}\right) ;{ }^{1} \mathrm{H} \mathrm{NMR}\left(300 \mathrm{MHz}, \mathrm{CDCl}_{3}\right) \delta$ $9.13(\mathrm{bs}, 1 \mathrm{H}, \mathrm{NH}), 7.21\left(\mathrm{~d}, 1 \mathrm{H}, J_{H A r, H A r}=8.5 \mathrm{~Hz}, H_{\mathrm{Ar}}\right), 5.77\left(\mathrm{~d}, 1 \mathrm{H}, J_{1,2}=9.5 \mathrm{~Hz}, H 1\right), 5.74\left(\mathrm{~d}, 1 \mathrm{H}, J_{H A r, H A r}=8.5 \mathrm{~Hz}\right.$, $\left.H_{\mathrm{Ar}}\right), 5.32\left(\mathrm{t}, 1 \mathrm{H}, J_{3,2}=J_{3,4}=9.0 \mathrm{~Hz}, H 3\right), 5.03\left(\mathrm{dd}, 1 \mathrm{H}, J_{2,1}=9.5 \mathrm{~Hz}, J_{2,3}=9.0 \mathrm{~Hz}, H 2\right), 4.33\left(\mathrm{~d}, 1 \mathrm{H}, J_{6,6}=12.5 \mathrm{~Hz}\right.$, $H 6), 4.20\left(\mathrm{dd}, 1 \mathrm{H}, J_{6,6}=12.5 \mathrm{~Hz}\right.$ and $\left.J_{6,5}=3.0 \mathrm{~Hz}, H 6^{\prime}\right), 3.72-3.53(\mathrm{~m}, 2 \mathrm{H}, H 4, H 5), 2.05(\mathrm{~s}, 6 \mathrm{H}, 2 \mathrm{CH}), 1.93(\mathrm{~s}$, $\left.3 \mathrm{H}, \mathrm{CH}_{3}\right) ;{ }^{13} \mathrm{C}$ NMR $\left(75 \mathrm{MHz}, \mathrm{CDCl}_{3}\right) \delta 170.5(\mathrm{C}=\mathrm{O}), 169.9(\mathrm{C}=\mathrm{O}), 169.6(C=\mathrm{O}), 162.4(C \mathrm{qAr}), 150.3(C \mathrm{qAr}), 139.1$ $\left(\mathrm{CH}_{\mathrm{Ar}}\right), 104.0\left(\mathrm{CH}_{\mathrm{Ar}}\right), 80.5(C 1), 75.4(C 5), 73.6(C 3), 69.6(C 2), 62.7(C 6), 60.1(C 4), 21.0\left(\mathrm{CH}_{3}\right), 20.8\left(\mathrm{CH}_{3}\right), 20.5$ $\left(\mathrm{CH}_{3}\right) ; \mathrm{IR} v\left(\mathrm{film}, \mathrm{cm}^{-1}\right) 2960(=\mathrm{C}-\mathrm{H}), 2111\left(\mathrm{~N}_{3}\right), 1748(\mathrm{C}=\mathrm{O}), 1689(\mathrm{NH}-\mathrm{C}=\mathrm{O}) ;$ ESIHRMS $\mathrm{m} / z=426.1260[\mathrm{M}+\mathrm{H}]^{+}$. $\mathrm{C}_{16} \mathrm{H}_{19} \mathrm{~N}_{5} \mathrm{O} 9 \mathrm{~F}$ requires 426.1261 .

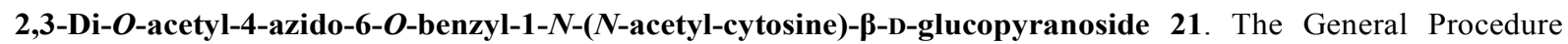
was followed using $8(50 \mathrm{mg}, 0.10 \mathrm{mmol}), 9(25 \mathrm{mg}, 0.16 \mathrm{mmol})$, BSA $(0.1 \mathrm{~mL}, 0.41 \mathrm{mmol})$, TMSOTf $(22 \mu \mathrm{L}, 0.12$ mmol), $4 \AA$ molecular sieves $(50 \mathrm{mg})$ in dry $\mathrm{MeCN}(1 \mathrm{~mL})$. The residue was purified by preparative TLC (Heptane/EtOAc 1:1) to afford product $21(28 \mathrm{mg}, 0.05 \mathrm{mmol}, 54 \%)$ as a colorless oil. $[\alpha]_{\mathrm{D}}{ }^{25}+47.5\left(c=1.0, \mathrm{CHCl}_{3}\right)$; ${ }^{1} \mathrm{H}$ NMR $\left(300 \mathrm{MHz}, \mathrm{CDCl}_{3}\right) \delta 7,67\left(\mathrm{~d}, 1 \mathrm{H}, J_{H A r, H A r}=7.5 \mathrm{~Hz}, H_{\mathrm{Ar}}\right), 7.47\left(\mathrm{~d}, 1 \mathrm{H}, J_{H A r, H A r}=7.5 \mathrm{~Hz}, H_{\mathrm{Ar}}\right) 7.36-7.27(\mathrm{~m}$, $\left.5 \mathrm{H}, H_{\mathrm{Ar}}\right), 5.99\left(\mathrm{~d}, 1 \mathrm{H}, J_{l, 2}=9.5 \mathrm{~Hz}, H 1\right), 5.32\left(\mathrm{dd}, 1 \mathrm{H}, J_{3,2}=9.5 \mathrm{~Hz}\right.$ and $\left.J_{3,4}=10.0 \mathrm{~Hz}, H 3\right), 5.03\left(\mathrm{t}, 1 \mathrm{H}, J_{2,3}=J_{2,1}=\right.$ $9.5 \mathrm{~Hz}, H 2), 4.57\left(\mathrm{~d}, 1 \mathrm{H}, J_{H, H}=12.0 \mathrm{~Hz}, \mathrm{CH} H_{2} \mathrm{Ph}\right), 4.52\left(\mathrm{~d}, 1 \mathrm{H}, J_{H, H}=12.0 \mathrm{~Hz}, \mathrm{CH}_{2} \mathrm{Ph}\right), 3.94\left(\mathrm{t}, 1 \mathrm{H}, J_{4,3}=J_{4,5}=10.0\right.$ $\mathrm{Hz}, H 6), 3.80-3.67(\mathrm{~m}, 2 \mathrm{H}, H 6), 3.60\left(\mathrm{ddd}, 1 \mathrm{H}, J_{5,4}=10.0 \mathrm{~Hz}\right.$ and $\left.J_{5,6}=3.5 \mathrm{~Hz}, J_{5,6^{\prime}}=2.0 \mathrm{~Hz}, H 5\right), 2.23(\mathrm{~s}, 3 \mathrm{H}$, $\left.\mathrm{NHCOCH}_{3}\right), 2.08\left(\mathrm{~s}, 3 \mathrm{H}, \mathrm{OCOCH}_{3}\right), 1.92\left(\mathrm{~s}, 3 \mathrm{H}, \mathrm{OCOCH}_{3}\right) ;{ }^{13} \mathrm{C} \mathrm{NMR}(75 \mathrm{MHz}, \mathrm{MeOD}) \delta 170.0(C=\mathrm{O}), 169.7$ $(C=\mathrm{O}), 163.1\left(C \mathrm{q}_{\mathrm{Ar}}\right), 155.3\left(C \mathrm{q}_{\mathrm{Ar}}\right), 144.5\left(C \mathrm{H}_{\mathrm{Ar}}\right), 137.6\left(C \mathrm{q}_{\mathrm{Ar}}\right), 128.7\left(C \mathrm{H}_{\mathrm{Ar}}\right), 128.2\left(C \mathrm{H}_{\mathrm{Ar}}\right), 128.1\left(C \mathrm{H}_{\mathrm{Ar}}\right), 98.0$ $\left(C \mathrm{H}_{\mathrm{Ar}}\right), 81.5(C 1), 77.3(C 5), 73.9\left(\mathrm{CH}_{2} \mathrm{Ph}\right), 73.5(C 2), 71.0(C 3), 68.2(C 6), 59.7(C 4), 25.2\left(\mathrm{COCH}_{3}\right), 20.8\left(\mathrm{COCH}_{3}\right)$, $20.6\left(\mathrm{COCH}_{3}\right) ; \mathrm{IR} v\left(\mathrm{film}, \mathrm{cm}^{-1}\right) 3148(=\mathrm{C}-\mathrm{H}), 2110\left(\mathrm{~N}_{3}\right), 1754(\mathrm{C}=\mathrm{O}), 1667(\mathrm{NH}-\mathrm{C}=\mathrm{O})$; ESIHRMS $m / z=515.1878$ $[\mathrm{M}+\mathrm{H}]^{+} . \mathrm{C}_{23} \mathrm{H}_{27} \mathrm{~N}_{6} \mathrm{O}_{8}$ requires 515.1890 .

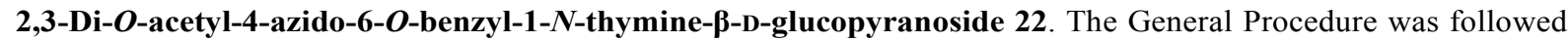
using 8 (581 mg, $1.2 \mathrm{mmol})$, Thymine $10(267 \mathrm{mg}, 2.1 \mathrm{mmol})$, BSA (1.3 mL, $5.29 \mathrm{mmol})$, TMSOTf (1.6 mL, 1.9 $\mathrm{mmol})$ in dry $\mathrm{MeCN}(25 \mathrm{~mL})$. The residue was purified by flash chromatography on silica gel (Heptane/EtOAc 70:30 to $60: 40)$ to afford product $22(496 \mathrm{mg}, 77 \%)$ as a white powder. $[\alpha]_{\mathrm{D}}{ }^{25}+11.1\left(c=1.0, \mathrm{CHCl}_{3}\right) ;{ }^{1} \mathrm{H} \mathrm{NMR}(300 \mathrm{MHz}$, $\left.\mathrm{CDCl}_{3}\right) \delta 7.42-7.25\left(\mathrm{~m}, 5 \mathrm{H}, H_{\mathrm{Ar}}\right), 7.11\left(\mathrm{~d}, 1 \mathrm{H}, J_{H A r, C H 3}=1.0 \mathrm{~Hz}, H_{\mathrm{Ar}}\right), 5.79\left(\mathrm{~d}, 1 \mathrm{H}, J_{1,2}=9.5 \mathrm{~Hz}, H 1\right), 5.29(\mathrm{dd}, 1 \mathrm{H}$, $J_{3,2}=9.5 \mathrm{~Hz}$ and $\left.J_{3,4}=10.0 \mathrm{~Hz}, H 3\right), 5.07\left(\mathrm{t}, 1 \mathrm{H}, J_{2, I}=J_{2,3}=9.5 \mathrm{~Hz}, H 2\right), 4.59\left(\mathrm{~d}, 1 \mathrm{H}, J_{H, H}=12.0 \mathrm{~Hz}, \mathrm{C} H_{2} \mathrm{Ph}\right), 4.52(\mathrm{~d}$, $\left.1 \mathrm{H}, J_{H, H}=12.0 \mathrm{~Hz}, \mathrm{CH}_{2} \mathrm{Ph}\right), 3.90\left(\mathrm{t}, 1 \mathrm{H}, J_{4,3}=J_{4,5}=10.0 \mathrm{~Hz}, H 4\right), 3.79-3.66\left(\mathrm{~m}, 2 \mathrm{H}, H 6, H 6\right.$ '), $2.08\left(\mathrm{~s}, 3 \mathrm{H}, \mathrm{C}(\mathrm{O}) \mathrm{CH} H_{3}\right)$, $1.95\left(\mathrm{~s}, 3 \mathrm{H}, \mathrm{C}(\mathrm{O}) \mathrm{CH}_{3}\right), 1.91\left(\mathrm{~d}, 3 \mathrm{H}, J_{\mathrm{CH} 3, \mathrm{HAr}}=1.0 \mathrm{~Hz}, \mathrm{C}_{\mathrm{Ar}} \mathrm{CH}_{3}\right) ;{ }^{13} \mathrm{C} \mathrm{NMR}\left(75 \mathrm{MHz}, \mathrm{CDCl}_{3}\right) \delta 169.9(\mathrm{C}=\mathrm{O}), 169.8$ $(C=\mathrm{O}), 163.5\left(C \mathrm{q}_{\mathrm{Ar}}\right), 150.6\left(C \mathrm{q}_{\mathrm{Ar}}\right), 137.6\left(C \mathrm{q}_{\mathrm{Ar}}\right), 134.6\left(C \mathrm{H}_{\mathrm{Ar}}\right), 128.7\left(C \mathrm{H}_{\mathrm{Ar}}\right), 128.2\left(C \mathrm{H}_{\mathrm{Ar}}\right), 128.0\left(C \mathrm{H}_{\mathrm{Ar}}\right), 112.3$ $\left(C \mathrm{q}_{\mathrm{Ar}}\right), 80.2(C 1), 76.9(C 5), 73.8\left(\mathrm{CH}_{2} \mathrm{Ph}\right), 73.7(C 3), 69.8(C 2), 68.2(C 6), 59.7(C 4), 20.8\left(\mathrm{C}(\mathrm{O}) C \mathrm{H}_{3}\right), 20.6$ $\left(\mathrm{C}(\mathrm{O}) \mathrm{CH}_{3}\right), 12.6\left(\mathrm{CArCH}_{3}\right) ; \mathrm{IR} v\left(\mathrm{film}, \mathrm{cm}^{-1}\right) 3675(\mathrm{~N}-\mathrm{H}), 2988(=\mathrm{C}-\mathrm{H}), 2901\left(\mathrm{CH}_{2}\right), 2111\left(\mathrm{~N}_{3}\right), 1754(\mathrm{C}=\mathrm{O}), 1697$ $(\mathrm{NH}-\mathrm{C}=\mathrm{O})$; ESIHRMS $m / z=487.1783[\mathrm{M}+\mathrm{H}]^{+}$. $\mathrm{C}_{22} \mathrm{H}_{26} \mathrm{~N}_{5} \mathrm{O}_{8}$ requires 487.1781 .

2,3-Di- $\boldsymbol{O}$-acetyl-4-azido-6- $\boldsymbol{O}$-benzyl-1- $\mathrm{N}$-(5-fluoro-uracil)- $\boldsymbol{\beta}$-D-glucopyranoside 23. A mixture of 5-fluoro-uracil 11 (42 mg, $0.31 \mathrm{mmol}, 1.5$ eq.), hexamethyldisilazane ( $77 \mu \mathrm{L}, 0.37 \mathrm{mmol}, 1.8$ eq.) and saccharine ( $3 \mathrm{mg}, 0.01 \mathrm{mmol}$, $6.5 \mathrm{~mol} \%)$ in anhydrous $\mathrm{MeCN}(1.5 \mathrm{~mL})$ was refluxed for $30 \mathrm{~min}$ under inert atmosphere. $8(100 \mathrm{mg}, 0.21 \mathrm{mmol}, 1$ eq.) and TMSOTf ( $56 \mu \mathrm{L}, 0.37 \mathrm{mmol}, 1.5$ eq. $)$ were then added and the resulting mixture was refluxed for $6 \mathrm{~h}$, cooled to room temperature, neutralized with saturated aqueous sodium bicarbonate $(3 \mathrm{~mL})$ and extracted with $\mathrm{CH}_{2} \mathrm{Cl}_{2}(8$ $\mathrm{mL}$ ). The organic extract was dried over anhydrous sodium sulfate, filtered and concentrated under reduced pressure. The residue was purified by flash chromatography (Heptane/EtOAc 90:10 to 50:50) to afford the clean product 23

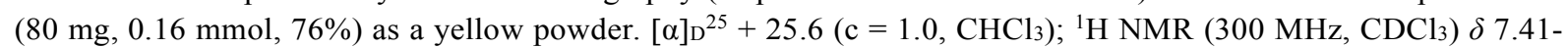
$7.26\left(\mathrm{~m}, 6 \mathrm{H}, H_{\mathrm{Ar}}\right), 5.75\left(\mathrm{dd}, 1 \mathrm{H}, J_{l, 2}=9.5 \mathrm{~Hz}\right.$ and $\left.J_{l, H A r}=1.5 \mathrm{~Hz}, H 1\right), 5.30\left(\mathrm{t}, 1 \mathrm{H}, J_{3,2}=J_{3,4}=9.5 \mathrm{~Hz}, H 3\right), 4.97(\mathrm{t}$, $\left.1 \mathrm{H}, J_{2,3}=J_{2, I}=9.5 \mathrm{~Hz}, H 2\right), 4.58\left(\mathrm{~d}, 1 \mathrm{H}, J_{H, H}=12.0 \mathrm{~Hz}, \mathrm{C} H_{2} \mathrm{Ph}\right), 4.53\left(\mathrm{~d}, 1 \mathrm{H}, J_{H, H}=12.0 \mathrm{~Hz}, \mathrm{CH} \mathrm{Ph}_{2}\right), 3.89(\mathrm{t}, 1 \mathrm{H}$, $\left.J_{4,3}=J_{4,5}=10.0 \mathrm{~Hz}, H 4\right), 3.80-3.66\left(\mathrm{~m}, 2 \mathrm{H}, H 6, H 6^{\prime}\right), 3.59\left(\mathrm{dt}, 1 \mathrm{H}, J_{5,4}=10.0 \mathrm{~Hz}, J_{5,6}=5.0 \mathrm{~Hz}, J_{5,6^{\prime}}=2.0 \mathrm{~Hz}, H 5\right)$, 
$2.09(\mathrm{~s}, 3 \mathrm{H}, \mathrm{CH} 3), 1.97\left(\mathrm{~s}, 3 \mathrm{H}, \mathrm{CH}_{3}\right) ;{ }^{13} \mathrm{C} \mathrm{NMR}\left(75 \mathrm{MHz}, \mathrm{CDCl}_{3}\right) \delta 170.0(\mathrm{C}=\mathrm{O}), 169.7(\mathrm{C}=\mathrm{O}), 156.5\left(\mathrm{~d}, J_{\mathrm{C}-\mathrm{F}}=27\right.$ $\left.\mathrm{Hz}, C \mathrm{q}_{\mathrm{Ar}}\right), 149.1\left(C \mathrm{q}_{\mathrm{Ar}}\right), 142.6\left(C \mathrm{q}_{\mathrm{Ar}}\right), 139.4\left(C \mathrm{q}_{\mathrm{Ar}}\right), 137.4\left(C \mathrm{q}_{\mathrm{Ar}}\right), 128.7\left(C \mathrm{H}_{\mathrm{Ar}}\right), 128.3\left(C \mathrm{H}_{\mathrm{Ar}}\right), 123.5\left(\mathrm{~d}, J_{\mathrm{C}-\mathrm{F}}=34 \mathrm{~Hz}\right.$, $\left.\mathrm{CH}_{\mathrm{Ar}}\right), 80.9(C 1), 77.0(C 5), 73.8\left(\mathrm{CH}_{2} \mathrm{Ph}\right), 73.3(C 3), 69.9(C 2), 68.1(C 6), 59.5(C 4), 20.8\left(\mathrm{C}(\mathrm{O}) C \mathrm{H}_{3}\right), 20.5$ $\left(\mathrm{C}(\mathrm{O}) \mathrm{CH}_{3}\right) ; \mathrm{IR} v\left(\right.$ film, $\left.\mathrm{cm}^{-1}\right) 3089(\mathrm{NH}), 2112\left(\mathrm{~N}_{3}\right), 1710(\mathrm{C}=\mathrm{O}), 1670(\mathrm{NH}-\mathrm{C}=\mathrm{O}) ;$ ESIHRMS $\mathrm{m} / z=514.1348$ $[\mathrm{M}+\mathrm{Na}]^{+} . \mathrm{C}_{21} \mathrm{H}_{22} \mathrm{~N}_{5} \mathrm{O}_{8} \mathrm{FNa}$ requires 514.1350 .

2,3-Di- $O$-acetyl-4-azido-6- $O$-benzyl-1- $N$-(4- $N$-benzoyl5-methyl-cytosine)- $\beta$-D-glucopyranoside 24 . The General Procedure was followed using 8 (45 mg, $0.092 \mathrm{mmol}), 12(34 \mathrm{mg}, 0.148 \mathrm{mmol})$, BSA (90 $\mu \mathrm{L}, 0.37 \mathrm{mmol})$, TMSOTf $(20 \mu \mathrm{L}, 0.11 \mathrm{mmol}), 4 \AA$ molecular sieves $(50 \mathrm{mg})$ in dry $\mathrm{MeCN}(0.9 \mathrm{~mL})$. The residue was purified by preparative TLC (Heptane/EtOAc 1:1) to afford product $24(36 \mathrm{mg}, 66 \%)$ as a yellow powder. $[\alpha]_{\mathrm{D}}{ }^{25}-12.7\left(c=1.2, \mathrm{CHCl}_{3}\right) ;{ }^{1} \mathrm{H}$ NMR $\left(300 \mathrm{MHz}, \mathrm{CDCl}_{3}\right) \delta 8.33-8.25\left(\mathrm{~m}, 2 \mathrm{H}, H_{\mathrm{Ar}}\right), 7.57-7.26\left(\mathrm{~m}, 9 \mathrm{H}, H_{\mathrm{Ar}}\right), 5.82\left(\mathrm{~d}, 1 \mathrm{H}, J_{l, 2}=9.5 \mathrm{~Hz}, H 1\right), 5.30(\mathrm{dd}$, $\left.1 \mathrm{H}, J_{3,2}=9.5 \mathrm{~Hz}, J_{3,4}=10.0 \mathrm{~Hz}, H 3\right), 5.10\left(\mathrm{t}, 1 \mathrm{H}, J_{2, I}=J_{2,3}=9.5 \mathrm{~Hz}, H 2\right), 4.60\left(\mathrm{~d}, 1 \mathrm{H}, J_{H, H}=12.0 \mathrm{~Hz}, \mathrm{C} H_{2} \mathrm{Ph}\right), 4.53$ (d, $\left.1 \mathrm{H}, J_{H, H}=12.0 \mathrm{~Hz}, \mathrm{CH} \mathrm{H}_{2} \mathrm{Ph}\right), 3.95\left(\mathrm{t}, 1 \mathrm{H}, J_{4,3}=J_{4,5}=10.0 \mathrm{~Hz}, H 4\right), 3.81-3.68(\mathrm{~m}, 2 \mathrm{H}, H 6, H 6$ '), 3.50 (ddd, $1 \mathrm{H}$, $\left.J_{5,4}=10.0 \mathrm{~Hz}, J_{5,6}=4.5 \mathrm{~Hz}, J_{5,6^{\prime}}=2.0 \mathrm{~Hz}, \mathrm{H5}\right), 2.10\left(\mathrm{~s}, 3 \mathrm{H}, \mathrm{CH}_{3}\right), 2.09\left(\mathrm{~s}, 3 \mathrm{H}, \mathrm{COCH}_{3}\right), 1.96\left(\mathrm{~s}, 3 \mathrm{H}, \mathrm{COCH}_{3}\right) ;{ }^{13} \mathrm{C}$ NMR $\left(75 \mathrm{MHz}, \mathrm{CDCl}_{3}\right) \delta 180.0(C=\mathrm{O}), 170.0(C=\mathrm{O}), 169.7(C=\mathrm{O}), 159.2\left(C \mathrm{q}_{\mathrm{Ar}}\right), 148.2\left(C \mathrm{q}_{\mathrm{Ar}}\right), 137.6\left(C \mathrm{q}_{\mathrm{Ar}}\right), 137.0$ $\left(C \mathrm{qAr}_{\mathrm{Ar}}\right), 135.9\left(C \mathrm{H}_{\mathrm{Ar}}\right), 132.9\left(C \mathrm{H}_{\mathrm{Ar}}\right), 130.2\left(C \mathrm{H}_{\mathrm{Ar}}\right), 128.7\left(C \mathrm{H}_{\mathrm{Ar}}\right), 128.4\left(C \mathrm{H}_{\mathrm{Ar}}\right), 128.2\left(C \mathrm{H}_{\mathrm{Ar}}\right), 128.0\left(C \mathrm{H}_{\mathrm{Ar}}\right), 113.1$ (CqAr), 80.7 (C1), $77.0(C 5), 73.8\left(\mathrm{CH}_{2} \mathrm{Ph}\right), 73.7(C 3), 69.9(C 2), 68.2(C 6), 59.6(C 4), 20.8\left(\mathrm{C}(\mathrm{O}) C \mathrm{H}_{3}\right), 20.6$ $\left(\mathrm{C}(\mathrm{O}) \mathrm{CH}_{3}\right), 13.9\left(\mathrm{CH}_{3}\right)$; IR $v\left(\right.$ film, $\left.\mathrm{cm}^{-1}\right) 2109\left(\mathrm{~N}_{3}\right), 1753(\mathrm{C}=\mathrm{O}), 1709(\mathrm{C}=\mathrm{O}) 1656(\mathrm{NH}-\mathrm{C}=\mathrm{O})$; ESIHRMS $m / z=$ $591.2208[\mathrm{M}+\mathrm{H}]^{+}$. $\mathrm{C}_{29} \mathrm{H}_{31} \mathrm{~N}_{6} \mathrm{O}_{8}$ requires 591.2203.

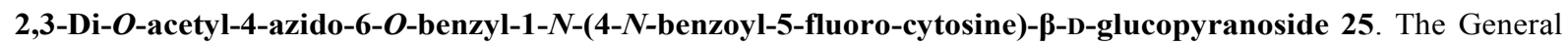
Procedure was followed using 8 (1.49 g, $3.05 \mathrm{mmol}), 13(1.14 \mathrm{~g}, 4.89 \mathrm{mmol})$, BSA (2.27 mL, $9.16 \mathrm{mmol})$, TMSOTf $(0.66 \mathrm{~mL}, 3.66 \mathrm{mmol})$ in dry $\mathrm{MeCN}(30 \mathrm{~mL})$. The residue was purified by preparative HPLC, gradient from 30 to $100 \% \mathrm{MeCN}$ in $15 \mathrm{~min}$, to afford product $25(918 \mathrm{mg}, 1.54 \mathrm{mmol}, 50 \%)$ as a yellow powder. $[\alpha]_{\mathrm{D}}{ }^{20}+22.9(c=0.84$, $\left.\mathrm{CHCl}_{3}\right) ;{ }^{1} \mathrm{H}$ NMR $\left(300 \mathrm{MHz}, \mathrm{CDCl}_{3}\right) \delta 8.29\left(\mathrm{~d}, 2 \mathrm{H}, J_{H A r, H A r}=7.0 \mathrm{~Hz}, H_{\mathrm{Ar}}\right), 7.57-7.43\left(\mathrm{~m}, 4 \mathrm{H}, H_{\mathrm{Ar}}\right), 7.42-7.30(\mathrm{~m}, 5 \mathrm{H}$, $\left.H_{\mathrm{Ar}}\right), 5.79\left(\mathrm{~d}, 1 \mathrm{H}, J_{l, 2}=9.0 \mathrm{~Hz}, H 1\right), 5.32\left(\mathrm{t}, 1 \mathrm{H}, J_{3,2}=J_{3,4}=9.5 \mathrm{~Hz}, H 3\right), 5.00\left(\mathrm{t}, 1 \mathrm{H}, J_{2,3}=J_{2, I}=9.5 \mathrm{~Hz}, H 2\right), 4.61(\mathrm{~d}$, $\left.1 \mathrm{H}, J_{H, H}=12.0 \mathrm{~Hz}, \mathrm{C} H_{2} \mathrm{Ph}\right), 4.56\left(\mathrm{~d}, 1 \mathrm{H}, J_{H, H}=12.0 \mathrm{~Hz}, \mathrm{CH}_{2} \mathrm{Ph}\right), 3.94\left(\mathrm{t}, 1 \mathrm{H}, J_{4,3}=J_{4,5}=10.0 \mathrm{~Hz}, H 4\right), 3.81-3.71(\mathrm{~m}$, $2 \mathrm{H}, H 6, H 6$ '), 3.59 (d, $\left.1 \mathrm{H}, J_{5,4}=10.0 \mathrm{~Hz}, H 5\right), 2.12(\mathrm{~s}, 3 \mathrm{H}, \mathrm{CH}), 2.00\left(\mathrm{~s}, 3 \mathrm{H}, \mathrm{CH}_{3}\right) ;{ }^{13} \mathrm{C} \mathrm{NMR}\left(75 \mathrm{MHz}, \mathrm{CDCl}_{3}\right) \delta$ $170.1(C=\mathrm{O}), 169.7(C=\mathrm{O}), 152.2\left(C \mathrm{q}_{\mathrm{Ar}}\right), 147.0\left(C \mathrm{q}_{\mathrm{Ar}}\right), 141.5\left(C \mathrm{q}_{\mathrm{Ar}}\right), 139.5\left(C \mathrm{q}_{\mathrm{Ar}}\right), 137.5\left(C \mathrm{q}_{\mathrm{Ar}}\right), 133.5\left(C \mathrm{H}_{\mathrm{Ar}}\right), 130.4$ $\left(C \mathrm{H}_{\mathrm{Ar}}\right), 128.8\left(\mathrm{CH}_{\mathrm{Ar}}\right), 128.6\left(\mathrm{CH}_{\mathrm{Ar}}\right), 128.3\left(\mathrm{CH}_{\mathrm{Ar}}\right), 128.1\left(\mathrm{CH}_{\mathrm{Ar}}\right), 81.1(C 1), 77.1(C 5), 73.9\left(C \mathrm{H}_{2} \mathrm{Ph}\right), 73.3(C 3), 70.1$ (C2), $68.1(C 6), 59.5(C 4), 20.8\left(\mathrm{C}(\mathrm{O}) \mathrm{CH}_{3}\right), 20.6\left(\mathrm{C}(\mathrm{O}) \mathrm{CH}_{3}\right)$; IR v (film, $\left.\mathrm{cm}^{-1}\right) 3089(=\mathrm{C}-\mathrm{H}), 2111\left(\mathrm{~N}_{3}\right), 1753(\mathrm{C}=\mathrm{O})$, $1672(\mathrm{NH}-\mathrm{C}=\mathrm{O})$; ESIHRMS $m / z=595.1951[\mathrm{M}+\mathrm{H}]^{+} . \mathrm{C}_{28} \mathrm{H}_{28} \mathrm{~N}_{6} \mathrm{O}_{8} \mathrm{~F}$ requires 595.1953 .

2,3-Di- $\boldsymbol{O}$-acetyl-4-azido-6- $\boldsymbol{O}$-benzyl-1- $\boldsymbol{N}$-uracil- $\boldsymbol{\beta}$-D-glucopyranoside 26 . The General Procedure was followed using 8 (400 mg, $0.82 \mathrm{mmol}), 14$ (147 mg, $1.31 \mathrm{mmol})$, BSA (0.80 mL, $3.28 \mathrm{mmol})$, TMSOTf (0.18 mL, $0.98 \mathrm{mmol})$ in dry $\mathrm{MeCN}(8.2 \mathrm{~mL})$. The residue was purified by flash chromatography (Heptane/EtOAc 90:10 to 50:50) to afford product $26(314 \mathrm{mg}, 0.66 \mathrm{mmol}, 81 \%)$ as a yellow powder. $[\alpha]_{\mathrm{D}}^{25}+20.6\left(c=1.0, \mathrm{CHCl}_{3}\right) ;{ }^{1} \mathrm{H} \mathrm{NMR}(300 \mathrm{MHz}$, $\left.\mathrm{CDCl}_{3}\right) \delta 8.78(\mathrm{bs}, 1 \mathrm{H}, \mathrm{N} H), 7.43-7.29\left(\mathrm{~m}, 6 \mathrm{H}, H_{\mathrm{Ar}}\right), 5.81\left(\mathrm{~d}, 1 \mathrm{H}, J_{l, 2}=9.0 \mathrm{~Hz}, H 1\right), 5.80\left(\mathrm{~d}, 1 \mathrm{H}, J_{H A r, H A r}=8.0 \mathrm{~Hz}\right.$, $\left.H_{\mathrm{Ar}}\right), 5.33\left(\mathrm{t}, 1 \mathrm{H}, J_{3,2}=J_{3,4}=9.5 \mathrm{~Hz}, H 3\right), 5.07\left(\mathrm{dd}, 1 \mathrm{H}, J_{2,1}=9.0 \mathrm{~Hz}, J_{2,3}=9.5 \mathrm{~Hz}, H 2\right), 4.62\left(\mathrm{~d}, 1 \mathrm{H}, J_{H, H}=12.0 \mathrm{~Hz}\right.$, $\left.\mathrm{CH}_{2} \mathrm{Ph}\right), 4.55\left(\mathrm{~d}, 1 \mathrm{H}, J_{H, H}=12.0 \mathrm{~Hz}, \mathrm{C} H_{2} \mathrm{Ph}\right), 3.94\left(\mathrm{dd}, 1 \mathrm{H}, J_{4,3}=9.5 \mathrm{~Hz}, J_{4,5}=10.0 \mathrm{~Hz}, H 4\right), 3.82-3.70(\mathrm{~m}, 2 \mathrm{H}, H 6$, $H 6$ '), $3.61\left(\mathrm{ddd}, 1 \mathrm{H}, J_{5,4}=10.0 \mathrm{~Hz}, J_{5,6}=3.0 \mathrm{~Hz}, J_{5,6}=2.0 \mathrm{~Hz}, H 5\right), 2.13$ (s, 3H, CH$), 1.99$ (s, $\left.3 \mathrm{H}, \mathrm{CH}_{3}\right) ;{ }^{13} \mathrm{C} \mathrm{NMR}$ $\left(75 \mathrm{MHz}, \mathrm{CDCl}_{3}\right) \delta 169.9(C=\mathrm{O}), 169.7(C=\mathrm{O}), 162.5\left(C \mathrm{q}_{\mathrm{Ar}}\right), 150.3\left(C \mathrm{q}_{\mathrm{Ar}}\right), 139.4\left(C \mathrm{H}_{\mathrm{Ar}}\right), 137.5\left(C \mathrm{q}_{\mathrm{Ar}}\right), 128.7\left(C \mathrm{H}_{\mathrm{Ar}}\right)$, $128.3\left(C \mathrm{H}_{\mathrm{Ar}}\right), 128.0\left(\mathrm{CH}_{\mathrm{Ar}}\right), 103.8\left(\mathrm{CH}_{\mathrm{Ar}}\right), 80.6(C 1), 77.0(C 5), 73.9\left(\mathrm{CH}_{2} \mathrm{Ph}\right), 73.5(C 3), 69.8(C 2), 68.2(C 6), 59.6$ $(C 4), 20.8\left(\mathrm{CH}_{3}\right), 20.6\left(\mathrm{CH}_{3}\right)$; IR $v\left(\right.$ film, $\left.\mathrm{cm}^{-1}\right) 2109\left(\mathrm{~N}_{3}\right), 1752(\mathrm{C}=\mathrm{O}), 1688(\mathrm{NH}-\underline{\mathrm{C}=\mathrm{O}})$; ESIHRMS $m / z=474.1626$ $[\mathrm{M}+\mathrm{H}]^{+} . \mathrm{C}_{21} \mathrm{H}_{24} \mathrm{~N}_{5} \mathrm{O}_{8}$ requires 474.1625 .

2,3,6-Tri- $\boldsymbol{O}$-acetyl-4-azido-1- $\boldsymbol{N}$-( $\boldsymbol{N}$-tert-butyloxycarbonyl--cytosine)- $\boldsymbol{\beta}$-D-glucopyranoside $\mathbf{2 7}$. To a stirred solution of 15 (40 mg, $0.09 \mathrm{mmol})$ in $\mathrm{CH}_{2} \mathrm{Cl}_{2}(1 \mathrm{~mL})$ was added di-tert-butyl-dicarbonate ( $40 \mu \mathrm{L}, 0.17 \mathrm{mmol}, 2$ eq.), triethylamine (12 $\mu \mathrm{L}, 0.09 \mathrm{mmol}, 1$ eq.) and 4-dimethylaminopyridine (10.5 mg, $0.09 \mathrm{mmol}, 1$ eq.). The resulting mixture was stirred at room temperature for $4 \mathrm{~h}$ and then concentrated under reduced pressure. The residue was purified by flash chromatography on silica gel $\left(\mathrm{CH}_{2} \mathrm{Cl}_{2} / \mathrm{MeOH} 99: 1\right.$ to 98:2) to afford product 27 (29 $\mathrm{mg}, 0.06 \mathrm{mmol}$, $64 \%)$ as a yellow powder. $[\alpha]_{\mathrm{D}}^{25}+41.7\left(c=1.2, \mathrm{CHCl}_{3}\right) ;{ }^{1} \mathrm{H} \mathrm{NMR}\left(300 \mathrm{MHz}, \mathrm{CDCl}_{3}\right) \delta 7.62\left(\mathrm{~d}, 1 \mathrm{H}, J_{H A r, H A r}=7.5\right.$ $\left.\mathrm{Hz}, H_{\mathrm{Ar}}\right), 7.30\left(\mathrm{~d}, 1 \mathrm{H}, J_{H A r, H A r}=7.5 \mathrm{~Hz}, H_{\mathrm{Ar}}\right), 6.05\left(\mathrm{~d}, 1 \mathrm{H}, J_{1,2}=9.5 \mathrm{~Hz}, H 1\right), 5.40\left(\mathrm{t}, 1 \mathrm{H}, J_{2,1}=J_{2,3}=9.5 \mathrm{~Hz}, H 2\right), 5.07$ $\left(\mathrm{t}, 1 \mathrm{H}, J_{3,2}=J_{3,4}=9.5 \mathrm{~Hz}, H 3\right), 4.39\left(\mathrm{~d}, 1 \mathrm{H}, J_{6,6^{\prime}}=12.5 \mathrm{~Hz}, H 6\right), 4.27\left(\mathrm{dd}, 1 \mathrm{H}, J_{6^{\prime}, 6}=12.5 \mathrm{~Hz}, J_{6^{\prime}, 5}=4.0 \mathrm{~Hz}, H 6^{\prime}\right)$, 3.76-3.67 (m, 2H, H4, H5), $2.11\left(\mathrm{~s}, 6 \mathrm{H}, \mathrm{OCOCH}_{3}\right), 1.97\left(\mathrm{~s}, 3 \mathrm{H}, \mathrm{OCOCH}_{3}\right), 1.51\left(\mathrm{~s}, 9 \mathrm{H}, \mathrm{OC}(\mathrm{CH})_{3}\right) ;{ }^{13} \mathrm{C} \mathrm{NMR}(75$ $\left.\mathrm{MHz}, \mathrm{CDCl}_{3}\right) \delta 170.5(C=\mathrm{O}), 170.2(C=\mathrm{O}), 169.5(C=\mathrm{O}), 143.8\left(C \mathrm{H}_{\mathrm{Ar}}\right), 96.3\left(C \mathrm{H}_{\mathrm{Ar}}\right), 83.7(C \mathrm{q}), 81.3(C 1), 75.5(C 4)$, 73.5 (C3), $70.7(\mathrm{C} 2), 62.7(C 6), 60.2(C 5), 28.2\left(\mathrm{C}\left(\mathrm{CH}_{3}\right)_{3}\right), 20.9\left(\mathrm{COCH}_{3}\right), 20.8\left(\mathrm{COCH}_{3}\right), 20.6\left(\mathrm{COCH}_{3}\right)$; IR $v(\mathrm{film}$, 
$\left.\mathrm{cm}^{-1}\right) 2987(\mathrm{C}-\mathrm{H}), 2109\left(\mathrm{~N}_{3}\right), 1751(\mathrm{C}=\mathrm{O}), 1731(\mathrm{C}=\mathrm{O}), 1667(\mathrm{NH}-\mathrm{C}=\mathrm{O}), 1626(\mathrm{NH}-\mathrm{C}=\mathrm{O})$; ESIHRMS m/z = $525.1945[\mathrm{M}+\mathrm{H}]^{+} . \mathrm{C}_{21} \mathrm{H}_{29} \mathrm{~N}_{6} \mathrm{O}_{10}$ requires 525.1953 .

Methyl Boc-sarcosinyl- $O$-tert-butyl-D-serinate 29-D. To a stirred solution of $O$-tert-butyl-D-serine methyl ester (250 $\mathrm{mg}, 1.18 \mathrm{mmol}, 1$ eq.) and Boc-sarcosine $\left(290 \mathrm{mg}, 1.54 \mathrm{mmol}, 1.3\right.$ eq.) in dry $\mathrm{CH}_{2} \mathrm{Cl}_{2}(9.8 \mathrm{~mL})$ were added $4 \AA$ molecular sieves (400 mg), hydroxybenzotriazole $(239 \mathrm{mg}, 1.77 \mathrm{mmol}, 1.5$ eq. $)$ and 1-ethyl-3-(3dimethylaminopropyl)carbodiimide ( $452 \mathrm{mg}, 2.36 \mathrm{mmol}, 2$ eq.). The resulting mixture was cooled to $0{ }^{\circ} \mathrm{C}$ and $\mathrm{Et}_{3} \mathrm{~N}$ ( $0.49 \mathrm{~mL}, 3.54 \mathrm{mmol}, 3 \mathrm{eq}$.) was added. After being stirred overnight at room temperature, the mixture was diluted with aqueous saturated $\mathrm{NaHCO}_{3}(8 \mathrm{~mL})$. The aqueous phase was then extracted with EtOAc $(3 \times 10 \mathrm{~mL})$. The organic layers were combined, dried over $\mathrm{Na}_{2} \mathrm{SO}_{4}$, filtered and concentrated under vacuum. The residue was purified by flash chromatography on silica gel (Heptane/EtOAc 80:20 to 60:40) to afford product 29-D (367 g, 90\%) as a colorless oil. $[\alpha]_{\mathrm{D}}{ }^{25}-26.4\left(c=1.0, \mathrm{CHCl}_{3}\right) ;{ }^{1} \mathrm{H} \mathrm{NMR}\left(300 \mathrm{MHz}, \mathrm{CDCl}_{3}\right) \delta 4.69(\mathrm{~m}, 1 \mathrm{H}, H 2), 4.17-3.81(\mathrm{~m}, 2 \mathrm{H}, H 3), 3.80(\mathrm{dd}, 1 \mathrm{H}$, $\left.J_{I, I^{\prime}}=9.0 \mathrm{~Hz}, J_{I^{\prime}, I}=3.0 \mathrm{~Hz}, H 1\right), 3.72\left(\mathrm{~s}, 3 \mathrm{H}, \mathrm{OCH}_{3}\right), 3.53\left(\mathrm{dd}, 1 \mathrm{H}, J_{l, I}=9.0 \mathrm{~Hz}, J_{I^{\prime}, I}=3.0 \mathrm{~Hz}, H 1\right), 2.93(\mathrm{~s}, 3 \mathrm{H}$, $\left.\mathrm{NCH}_{3}\right), 1.46\left(\mathrm{~s}, 9 \mathrm{H}, \mathrm{CO}_{2} \mathrm{C}\left(\mathrm{CH}_{3}\right)_{3}\right), 1.10\left(\mathrm{~s}, 9 \mathrm{H}, \mathrm{OC}\left(\mathrm{CH}_{3}\right)_{3}\right) ;{ }^{13} \mathrm{C} \mathrm{NMR}\left(75 \mathrm{MHz}, \mathrm{CDCl}_{3}\right) \delta 62.1(\mathrm{C} 1), 53.4(\mathrm{C} 3), 52.8$

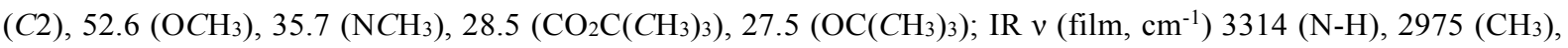
$2935\left(\mathrm{CH}_{2}\right), 1751(\mathrm{C}=\mathrm{O}), 1684(\mathrm{NH}-\mathrm{C}=\mathrm{O})$; ESIHRMS $m / z=347.2182[\mathrm{M}+\mathrm{H}]^{+} . \mathrm{C}_{16} \mathrm{H}_{31} \mathrm{~N}_{2} \mathrm{O}_{6}$ requires 347.2191.

Boc-sarcosinyl-O-tert-butyl-D-serine 30-D. To a stirred solution of 29-D (367 mg, $1.06 \mathrm{mmol}, 1$ eq.) in THF/ $\mathrm{H}_{2} \mathrm{O}$ mixture $(8.8 \mathrm{~mL} / 1.8 \mathrm{~mL} 5: 1)$, was added lithium hydroxide $(33 \mathrm{mg}, 1.38 \mathrm{mmol}, 1.3 \mathrm{eq}$.). The resulting mixture was stirred for $1 \mathrm{~h}$ at room temperature and then concentrated under vacuum until THF was evaporated. HCl $1 \mathrm{~N}$ was then added until the $\mathrm{pH}$ 2. The aqueous layer was extracted with EtOAc $(3 \times 10 \mathrm{~mL})$. The organic layers were combined, dried over $\mathrm{Na}_{2} \mathrm{SO}_{4}$ and concentrated under vacuum to afford the clean product 30-D (351 $\mathrm{mg}, 1.06 \mathrm{mmol}$, quantitative). The product is used without further purification. $[\alpha]_{\mathrm{D}}{ }^{25}-32.8\left(c=1.0, \mathrm{CHCl}_{3}\right) ;{ }^{1} \mathrm{H} \mathrm{NMR}\left(300 \mathrm{MHz}, \mathrm{CDCl}_{3}\right) \delta 6.90$ $\left(\mathrm{d}, 1 \mathrm{H}, J_{N H, H 2}=8.0 \mathrm{~Hz}, N \mathrm{H}\right), 4.68(\mathrm{~m}, 1 \mathrm{H}, H 2), 4.07-3.64\left(\mathrm{~m}, 2 \mathrm{H}, H 3, H 3\right.$ '), $3.85\left(\mathrm{dd}, 1 \mathrm{H}, J_{l, l},=9.0 \mathrm{~Hz}, J_{l, 2}=3.0 \mathrm{~Hz}\right.$, $\left.H 1), 3.55\left(\mathrm{dd}, 1 \mathrm{H}, J_{l, l^{\prime}}=9.0 \mathrm{~Hz}, J_{I^{\prime}, 2}=4.0 \mathrm{~Hz}, H 1^{\prime}\right), 2.92(\mathrm{~s}, 3 \mathrm{H}, \mathrm{NCH}), 1.44\left(\mathrm{~s}, 9 \mathrm{H}, \mathrm{CO}_{2} \mathrm{C}_{(\mathrm{CH}}\right)_{3}\right), 1.12(\mathrm{~s}, 9 \mathrm{H}$, $\left.\mathrm{OC}\left(\mathrm{CH}_{3}\right)_{3}\right) ;{ }^{13} \mathrm{C}$ NMR $\left(75 \mathrm{MHz}, \mathrm{CDCl}_{3}\right) \delta 173.7(\mathrm{C}=\mathrm{O}), 169.9(\mathrm{C}=\mathrm{O}), 81.2\left(\mathrm{CqCO}_{\mathrm{C}(\mathrm{CH}) 3}\right), 74.1(\mathrm{CqO} C(\mathrm{CH}) 3), 61.7$ $(C 1), 53.3(C 3), 52.7(C 2), 35.8\left(\mathrm{NCH}_{3}\right), 28.5\left(\mathrm{CO}_{2} \mathrm{C}\left(\mathrm{CH}_{3}\right)_{3}\right), 27.5\left(\mathrm{OC}\left(\mathrm{CH}_{3}\right)_{3}\right) ; \mathrm{IR} v\left(\mathrm{film}, \mathrm{cm}^{-1}\right) 3320(\mathrm{O}-\mathrm{H}), 2975$ $\left(\mathrm{CH}_{3}\right), 2935\left(\mathrm{CH}_{2}\right), 1739(\mathrm{C}=\mathrm{O}), 1670(\mathrm{NH}-\mathrm{C}=\mathrm{O})$; ESIHRMS $m / z=355.1842[\mathrm{M}+\mathrm{Na}]^{+} . \mathrm{C}_{15} \mathrm{H}_{28} \mathrm{~N}_{2} \mathrm{O}_{6} \mathrm{Na}$ requires 355.1845 .

Boc-sarcosinyl-O-tert-butyl-L-serine methyl ester 29-L. To a stirred solution of $O$-tert-butyl-L-serine methyl ester hydrochloride (250 mg, $1.18 \mathrm{mmol}, 1$ eq.) and Boc-sarcosine (290 mg, 1,55 mmol, 1.3 eq.) in dry $\mathrm{CH}_{2} \mathrm{Cl}_{2}(10 \mathrm{~mL})$ were added $4 \AA$ molecular sieves $(335 \mathrm{mg})$, hydroxybenzotriazole $(240 \mathrm{mg}, 1.77 \mathrm{mmol}, 1.5 \mathrm{eq}$.) and 1-ethyl-3-(3dimethylaminopropyl)carbodiimide ( $453 \mathrm{mg}, 0.47 \mathrm{mmol}, 2$ eq.). The resulting mixture was cooled to $0{ }^{\circ} \mathrm{C}$ and $\mathrm{Et} 3 \mathrm{~N}$ $(0.5 \mathrm{~mL}, 2.36 \mathrm{mmol}, 3$ eq. $)$ was added. After being stirred overnight at room temperature, the mixture was diluted with aqueous saturated $\mathrm{NaHCO}_{3}(6 \mathrm{~mL})$. Aqueous phase was then extracted with EtOAc $(3 \mathrm{x} 5 \mathrm{~mL})$. Organic layers were combined, dried over $\mathrm{Na}_{2} \mathrm{SO}_{4}$, filtered and concentrated under vacuum. The residue was purified by flash chromatography on silica gel (Heptane/EtOAc 50:50 to 30:70) to afford product 29-L (400 g, $1.16 \mathrm{mmol}, 98 \%$ ) as a colorless oil. $[\alpha]_{\mathrm{D}}{ }^{25}+32.9\left(c=0.82, \mathrm{CHCl}_{3}\right)$.

Boc-sarcosinyl-O-tert-butyl-L-serine 30-L. To a stirred solution of $\mathbf{2 9 - \mathrm { L }}\left(320 \mathrm{mg}, 0.92 \mathrm{mmol}, 1\right.$ eq.) in THF/ $\mathrm{H}_{2} \mathrm{O}$ mixture (7.7 mL/1.5 mL 5:1) was added lithium hydroxide (29 mg, $1.20 \mathrm{mmol}, 1.3 \mathrm{eq}$ ). The resulting mixture was stirred for $1 \mathrm{~h}$ at room temperature and then concentrated under vacuum until THF was evaporated. HCl $1 \mathrm{~N}$ was then added until $\mathrm{pH}$ 2. The aqueous layer was extracted with EtOAc $(3 \times 10 \mathrm{~mL})$. The organic layers were combined, dried over $\mathrm{Na}_{2} \mathrm{SO}_{4}$ and concentrated under vacuum to afford the clean product 30-L (305 $\mathrm{mg}, 0.92 \mathrm{mmol}$, quantitative). The product is used without further purification. $[\alpha] \mathrm{D}^{25}+27.3\left(c=1.0, \mathrm{CHCl}_{3}\right)$.

Peptidonucleoside 31-D. The solution 1 was prepared with $\mathrm{Na}(10 \mathrm{mg})$ in dry $\mathrm{MeOH}(2 \mathrm{~mL}, \mathrm{C}=0.22 \mathrm{M})$. To a stirred solution of the protected nucleoside $27(147 \mathrm{mg}, 0.28 \mathrm{mmol})$ in dry $\mathrm{MeOH}(5 \mathrm{~mL})$ was added the solution $1(0.26 \mathrm{~mL}$, $20 \mathrm{~mol} \%)$. The resulting mixture was stirred at room temperature for $1 \mathrm{~h}$ and then neutralized with Dowex ${ }^{\circledR} \mathrm{H}^{+}$, filtered on celite and concentrated under reduce pressure to afford clean product without further purification. The obtained product $(105 \mathrm{mg})$ was then hydrogenated at atmospheric pressure in the presence of $\mathrm{Pd}(\mathrm{OH})_{2}(40 \% \mathrm{w} / \mathrm{w}, 42$ $\mathrm{mg})$ in $\mathrm{MeOH}(2.6 \mathrm{~mL})$ for $12 \mathrm{~h}$. The resulting mixture was then filtered on celite ${ }^{\circledR}$ and concentrated under reduced pressure to afford the clean corresponding amine (94 mg). To a stirred solution of the latter in DMF (4 mL) was added the dipeptide (109 mg, $0.33 \mathrm{mmol}, 1.3$ eq.) and DIPEA (0.17 mL, $1.01 \mathrm{mmol}, 4$ eq.). After $1 \mathrm{~min}$, HATU (144 mg, $0.32 \mathrm{mmol}, 1.5$ eq.) was added and the resulting mixture was stirred at room temperature for $18 \mathrm{~h}$. Solvent was removed and the crude product was purified by flash chromatography on silica gel (EtOAc/EtOH 99:1 to 92:8) to afford product 31-D $(113 \mathrm{mg}, 0.16 \mathrm{mmol}, 65 \%)$ as a yellow powder; $[\alpha]_{\mathrm{D}}{ }^{25}+14.6(c=1.1, \mathrm{MeOH}) .{ }^{1} \mathrm{H} \mathrm{NMR}(500$ 
$\left.\mathrm{MHz}, \mathrm{CD}_{3} \mathrm{OD}\right)^{34} \delta 8.11\left(\mathrm{~d}, 1 \mathrm{H}, J_{H A r, H A r}=7.5 \mathrm{~Hz}, H_{\mathrm{Ar}}\right), 7.33-7.26\left(\mathrm{~m}, 1 \mathrm{H}, H_{\mathrm{Ar}}\right), 5.77\left(\mathrm{~d}, 1 \mathrm{H}, J_{I, 2}=9.0 \mathrm{~Hz}, H 1\right), 4.60-$ $4.49(\mathrm{~m}, 1 \mathrm{H}, H 7), 4.10-3.53(\mathrm{~m}, 10 \mathrm{H}, H 2, H 3, H 4, H 5, H 6, H 8, H 9), 3.03-2.83(\mathrm{~m}, 3 \mathrm{H}, \mathrm{NCH}), 1.56(\mathrm{~s}, 9 \mathrm{H}$, $\left.\mathrm{CO}_{2} \mathrm{C}\left(\mathrm{CH}_{3}\right)_{3}\right), 1.50-1.41\left(\mathrm{~m}, 9 \mathrm{H}, \mathrm{OC}\left(\mathrm{CH}_{3}\right)_{3}\right), 1.20\left(\mathrm{~s}, 9 \mathrm{H}, \mathrm{CO}_{2} \mathrm{C}\left(\mathrm{CH}_{3}\right)_{3}\right) ;{ }^{13} \mathrm{C} \mathrm{NMR}\left(75 \mathrm{MHz}, \mathrm{CD}_{3} \mathrm{OD}\right)^{35} \delta 172.1$ $(C=\mathrm{O}), 163.7(C \mathrm{q}), 157.0(C \mathrm{q}), 156.9(C \mathrm{q}), 151.9(C=\mathrm{O}), 144.9\left(\mathrm{CH}, C_{\mathrm{Ar}}\right), 96.1\left(\mathrm{CH}, C_{\mathrm{Ar}}\right), 85.2(C 1), 83.4\left(C\left(\mathrm{CH}_{3}\right)_{3}\right)$, $80.2(\mathrm{CH}), 75.8(\mathrm{CH}), 74.4\left(\mathrm{CH}_{2}\right), 73.9\left(\mathrm{C}_{\left.\left(\mathrm{CH}_{3}\right)_{3}\right),} 73.8\left(\mathrm{C}\left(\mathrm{CH}_{3}\right)_{3}\right), 71.6\left(\mathrm{CH}_{2}\right), 62.8\left(\mathrm{CH}_{2}\right), 56.1(C 7), 53.4(\mathrm{CH})\right.$,

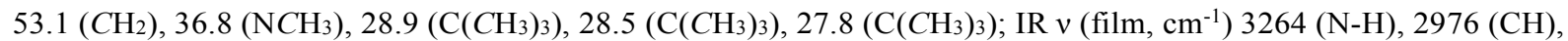
$2926(\mathrm{CH}), 1758(\mathrm{C}=\mathrm{O}), 1656(\mathrm{NH}-\mathrm{C}=\mathrm{O})$; ESIHRMS m/z $=687.3566[\mathrm{M}+\mathrm{H}]^{+} . \mathrm{C}_{30} \mathrm{H}_{51} \mathrm{~N}_{6} \mathrm{O}_{12}$ requires 687.3565.

Peptidonucleoside 32-D. To a stirred solution of 31-D (90 mg, $0.131 \mathrm{mmol}, 1$ eq.) in $\mathrm{CH}_{2} \mathrm{Cl}_{2} / \mathrm{MeOH}(2: 1 \mathrm{v} / \mathrm{v}, 1.3 \mathrm{~mL})$ was added a solution of $4 \mathrm{M} \mathrm{HCl}$ in dioxane $(0.23 \mathrm{~mL}, 0.92 \mathrm{mmol}, 7 \mathrm{eq}$.). The resulting mixture was stirred at room temperature for 2 days and then diluted with $\mathrm{H}_{2} \mathrm{O}$ and then neutralized with DOWEX ${ }^{\circledR}$ MONOSPHERE ${ }^{\circledR} 550 \mathrm{~A}(\mathrm{OH})$ anion exchange resin. The mixture was filtered on celite and then concentrated under vaccum. The crude product was purified by preparative TLC $\left(\mathrm{H}_{2} \mathrm{O} / \mathrm{EtOH} / \mathrm{EtOAc} 4: 4: 2, \mathrm{pH}\right.$ 9) to afford 32-D as a white powder $(21 \mathrm{mg}, 0.05 \mathrm{mmol}$, $37 \%) .[\alpha]_{\mathrm{D}^{25}}-31.3\left(c=1.1, \mathrm{H}_{2} \mathrm{O}\right) ;{ }^{1} \mathrm{H}$ NMR $\left(300 \mathrm{MHz}, \mathrm{D}_{2} \mathrm{O}\right) \delta 7.73\left(\mathrm{~d}, 1 \mathrm{H}, J_{H A r, H A r}=7.5 \mathrm{~Hz}, H_{\mathrm{Ar}}\right), 6.07(\mathrm{bs}, 1 \mathrm{H}$, $\left.H_{\mathrm{Ar}}\right), 5.64\left(\mathrm{~d}, 1 \mathrm{H}, J_{l, 2}=9.5 \mathrm{~Hz}, H 1\right), 4.45\left(\mathrm{t}, 1 \mathrm{H}, J_{7,8}=J_{7,8}=5.5 \mathrm{~Hz}, H 7\right), 3.94(\mathrm{~s}, 2 \mathrm{H}, H 9), 3.91-3.80(\mathrm{~m}, 1 \mathrm{H}, H 4)$, $3.86\left(\mathrm{~d}, 2 \mathrm{H}, J_{8,7}=5.5 \mathrm{~Hz}, H 8\right), 3.80-3.71(\mathrm{~m}, 3 \mathrm{H}, H 2, H 3, H 5), 3.68\left(\mathrm{dd}, 1 \mathrm{H}, J_{6,6}=12.5 \mathrm{~Hz}, J_{6,5}=1.5 \mathrm{~Hz}, H 6\right), 3.57$ $\left(\mathrm{dd}, 1 \mathrm{H}, J_{6,6}=12.5 \mathrm{~Hz}, J_{6^{\prime}, 5}=5.5 \mathrm{~Hz}, H 6^{\prime}\right), 2.74\left(\mathrm{~s}, 3 \mathrm{H}, \mathrm{NCH}_{3}\right) ;{ }^{13} \mathrm{C} \mathrm{NMR}\left(75 \mathrm{MHz}, \mathrm{D}_{2} \mathrm{O}\right) \delta 172.0(C=\mathrm{O}), 166.7$ $(C=\mathrm{O}), 166.0(C=\mathrm{O}), 157.9\left(C \mathrm{q}_{\mathrm{Ar}}\right), 141.7\left(C \mathrm{H}_{\mathrm{Ar}}\right), 97.0\left(C \mathrm{H}_{\mathrm{Ar}}\right), 83.3(C 1), 77.5(C 5), 73.7(C 3), 71.7(C 2), 61.0(C 8)$, 60.6 (C6), 55.9 (C7), $51.3(C 4), 49.4(C 9), 32.8\left(\mathrm{NCH}_{3}\right)$; IR v (film, cm $\left.{ }^{-1}\right) 3310(\mathrm{O}-\mathrm{H}), 3282(\mathrm{~N}-\mathrm{H}), 2976\left(\mathrm{CH}_{3}\right), 2933$ $\left(\mathrm{CH}_{2}\right), 1744(\mathrm{C}=\mathrm{O}), 1653(\mathrm{NH}-\mathrm{C}=\mathrm{O})$; ESIHRMS $m / z=431.3424[\mathrm{M}+\mathrm{H}]^{+} . \mathrm{C}_{16} \mathrm{H}_{28} \mathrm{~N}_{6} \mathrm{O}_{8}$ requires 431.1890.

Peptidonucleoside 31-L. The solution 1 was prepared with $\mathrm{Na}(10 \mathrm{mg})$ in dry $\mathrm{MeOH}(2 \mathrm{~mL}, \mathrm{C}=0.22 \mathrm{M})$. To a stirred solution of the protected nucleoside $27(503 \mathrm{mg}, 0.96 \mathrm{mmol})$ in dry $\mathrm{MeOH}(16 \mathrm{~mL})$ was added the solution 1 $(0.88 \mathrm{~mL}, 20 \mathrm{~mol} \%)$. The resulting mixture was stirred at room temperature for $1 \mathrm{~h}$ and then neutralized with Dowex ${ }^{\circledR}$ $\mathrm{H}^{+}$, filtered on celite and concentrated under reduce pressure to afford clean product without further purification. The obtained product $(355 \mathrm{mg})$ was then hydrogenolysed at atmospheric pressure in the presence of $\mathrm{Pd}(\mathrm{OH})_{2}-\mathrm{C}(40 \% \mathrm{w} / \mathrm{w}$, $142 \mathrm{mg})$ in $\mathrm{MeOH}(8.9 \mathrm{~mL})$ for $12 \mathrm{~h}$. The resulting mixture was then filtered on celite $\AA$ and concentrated under reduced pressure to afford the clean corresponding amine $(318 \mathrm{mg})$. To a stirred solution of the latter $(150 \mathrm{mg}, 0.40$ $\mathrm{mmol})$ in DMF $(6 \mathrm{~mL})$ was added the dipeptide $(174 \mathrm{mg}, 0.52 \mathrm{mmol}, 1.3$ eq. $)$ and DIPEA $(0.28 \mathrm{~mL}, 1.61 \mathrm{mmol}, 4$ eq.). After $1 \mathrm{~min}$, HATU (184 mg, $0.48 \mathrm{mmol}, 1.5$ eq.) was added and the resulting mixture was stirred at room temperature for $18 \mathrm{~h}$. Solvent was removed and the crude product was purified by flash chromatography on silica gel (EtOAc/EtOH 99:1 to 92:8) to afford product 31-L $(158 \mathrm{mg}, 0.23 \mathrm{mmol}, 57 \%)$ as a yellow powder. $[\alpha]_{\mathrm{D}^{20}}-99.0(c=$ $\left.0.34, \mathrm{H}_{2} \mathrm{O} / \mathrm{MeOH} 1: 1\right) ;{ }^{1} \mathrm{H}$ NMR $\left(500 \mathrm{MHz}, \mathrm{CD}_{3} \mathrm{OD}\right){ }^{34} \delta 8.15\left(\mathrm{~d}, 1 \mathrm{H}, J_{H A r, H A r}=7.5 \mathrm{~Hz}, H_{\mathrm{Ar}}\right), 7.37\left(\mathrm{~d}, 1 \mathrm{H}, J_{H A r, H A r}=\right.$ $\left.7.5 \mathrm{~Hz}, H_{\mathrm{Ar}}\right), 5.81\left(\mathrm{~d}, 1 \mathrm{H}, J_{1,2}=9.0 \mathrm{~Hz}, H 1\right), 4.60-4.49(\mathrm{~m}, 1 \mathrm{H}, H 7), 4.10-3.97(\mathrm{~m}, 2 \mathrm{H}, \mathrm{H} 9), 3.95\left(\mathrm{t}, 1 \mathrm{H}, J_{4,3}=J_{4,5}=\right.$ $10.0 \mathrm{~Hz}, H 4), 3.87-3.71(\mathrm{~m}, 2 \mathrm{H}, H 3, H 6), 3.71-3.58$ (m, 5H, H2, H5, H6', H8), 3.03-2.91 (bs, 3H, NCH3), 1.58 (s, $\left.9 \mathrm{H}, \mathrm{CO}_{2} \mathrm{C}\left(\mathrm{CH}_{3}\right)_{3}\right), 1.55-1.47\left(\mathrm{~m}, 9 \mathrm{H}, \mathrm{OC}\left(\mathrm{CH}_{3}\right)_{3}\right), 1.25\left(\mathrm{~s}, 9 \mathrm{H}, \mathrm{CO}_{2} \mathrm{C}\left(\mathrm{CH}_{3}\right)_{3}\right) ;{ }^{13} \mathrm{C} \mathrm{NMR}\left(75 \mathrm{MHz}, \mathrm{CD}_{3} \mathrm{OD}\right)^{35} \delta 173.4$ $(C=\mathrm{O}), 171.9(C=\mathrm{O}), 165.3(C \mathrm{q}), 158.6(C \mathrm{q}), 153.6(C=\mathrm{O}), 146.5\left(C \mathrm{H}_{\mathrm{Ar}}\right), 97.7\left(C \mathrm{H}_{\mathrm{Ar}}\right), 85.3(C 1), 83.4\left(C\left(\mathrm{CH}_{3}\right)_{3}\right)$, $81.8\left(\mathrm{C}_{\left.\left(\mathrm{CH}_{3}\right)_{3}\right),} 80.2(\mathrm{C5}), 75.8(\mathrm{C3}), 75.0\left(\mathrm{C}\left(\mathrm{CH}_{3}\right)_{3}\right), 74.5(\mathrm{C} 2), 62.8(\mathrm{C} 6), 58.6(\mathrm{C} 8), 55.7(\mathrm{C} 7), 53.2(\mathrm{C} 4), 52.9\right.$

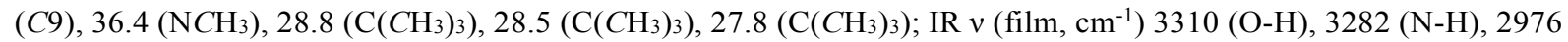
$\left(\mathrm{CH}_{3}\right), 2933\left(\mathrm{CH}_{2}\right), 1744(\mathrm{C}=\mathrm{O}), 1653(\mathrm{NH}-\mathrm{C}=\mathrm{O})$; ESIHRMS $m / z=687.3566[\mathrm{M}+\mathrm{H}]^{+} . \mathrm{C}_{30} \mathrm{H}_{51} \mathrm{~N}_{6} \mathrm{O}_{12}$ requires 687.3565 .

\footnotetext{
Analogue 32-L. To a stirred solution of 31-L (90 mg, $0.131 \mathrm{mmol}, 1$ eq.) in $\mathrm{CH}_{2} \mathrm{Cl}_{2} / \mathrm{MeOH}(2: 1 \mathrm{v} / \mathrm{v}, 1.3 \mathrm{~mL})$ was added a solution of $4 \mathrm{M} \mathrm{HCl}$ in dioxane $(0.23 \mathrm{~mL}, 0.92 \mathrm{mmol}, 7 \mathrm{eq}$.$) . The resulting mixture was stirred at room$ temperature for 2 days and then diluted with $\mathrm{H}_{2} \mathrm{O}$ and then neutralized with DOWEX ${ }^{\circledR}$ MONOSPHERE ${ }^{\circledR} 550 \mathrm{~A}(\mathrm{OH})$ anion exchange resin. The mixture was filtered on celite and then concentrated under vaccum. The crude product was purified by preparative TLC $\left(\mathrm{H}_{2} \mathrm{O} / \mathrm{EtOH} / \mathrm{EtOAc} 4: 4: 2, \mathrm{pH} 9\right)$ to afford clean product 32-L as a white powder (10.3 $\mathrm{mg}, 0.024 \mathrm{mmol}, 28 \%)$. $[\alpha]_{\mathrm{D}}{ }^{25}+278.3\left(c=1.0, \mathrm{H}_{2} \mathrm{O}\right) ;{ }^{1} \mathrm{H}$ NMR $\left(500 \mathrm{MHz}, \mathrm{D}_{2} \mathrm{O}\right) \delta 7.80\left(\mathrm{~d}, 1 \mathrm{H}, J_{H A r, H A r}=7.5 \mathrm{~Hz}\right.$, $\left.H_{\mathrm{Ar}}\right), 6.14\left(\mathrm{~d}, 1 \mathrm{H}, J_{H A r, H A r}=7.5 \mathrm{~Hz}, H_{\mathrm{Ar}}\right), 5.71\left(\mathrm{~d}, 1 \mathrm{H}, J_{1,2}=9.0 \mathrm{~Hz}, H 1\right), 4.52\left(\mathrm{t}, 1 \mathrm{H}, J_{7,8}=J_{7,8}=5.5 \mathrm{~Hz}, H 7\right), 3.99(\mathrm{t}$, $\left.1 \mathrm{H}, J_{4,3}=J_{4,5}=10.0 \mathrm{~Hz}, H 4\right), 3.92\left(\mathrm{~d}, 2 \mathrm{H}, J_{8,7}=5.5 \mathrm{~Hz}, H 8\right), 3.85\left(\mathrm{dd}, 1 \mathrm{H}, J_{3,2}=9.0 \mathrm{~Hz}\right.$ and $\left.J_{3,4}=10.0 \mathrm{~Hz}, H 3\right), 3.82$ $\left(\mathrm{t}, 1 \mathrm{H}, J_{2,3}=J_{2, I}=9.0 \mathrm{~Hz}, H 2\right), 3.80-3.70(\mathrm{~m}, 2 \mathrm{H}, H 5, H 6), 3.66-3.60(\mathrm{~m}, 3 \mathrm{H}, H 6, H 9), 2.54(\mathrm{~s}, 3 \mathrm{H}, \mathrm{NCH}) ;{ }^{13} \mathrm{C} \mathrm{NMR}$ $\left(75 \mathrm{MHz}, \mathrm{D}_{2} \mathrm{O}\right) \delta 172.2(C=\mathrm{O}), 171.1(C=\mathrm{O}), 166.1(C=\mathrm{O}), 158.0\left(C \mathrm{q}_{\mathrm{Ar}}\right), 141.8\left(C \mathrm{H}_{\mathrm{Ar}}\right), 97.1\left(C \mathrm{H}_{\mathrm{Ar}}\right), 83.1(C 1), 77.5$ (C5), 73.8 (C3), 71.7 (C2), 61.2 (C8), 60.7 (C6), 55.8 (C7), 51.4 (C4), 51.3 (C9), $33.8\left(\mathrm{NCH}_{3}\right)$; IR v (film, cm $\left.{ }^{-1}\right) 3310$ $(\mathrm{O}-\mathrm{H}), 3282(\mathrm{~N}-\mathrm{H}), 2976\left(\mathrm{CH}_{3}\right), 2933\left(\mathrm{CH}_{2}\right), 1744(\mathrm{C}=\mathrm{O}), 1653(\mathrm{NH}-\mathrm{C}=\mathrm{O})$; ESIHRMS $\mathrm{m} / z=431.1890[\mathrm{M}+\mathrm{H}]^{+}$. $\mathrm{C}_{16} \mathrm{H}_{27} \mathrm{~N}_{6} \mathrm{O} 8$ requires 431.1901 .
}

Peptidonucleoside 33. The same Procedure as described for 31-D was followed using 16 (315 mg, $0.72 \mathrm{mmol}$ ), solution $1(0.65 \mathrm{~mL}, \mathrm{C}=0.44 \mathrm{M}, 40 \mathrm{~mol} \%)$ in $\mathrm{MeOH}(10 \mathrm{~mL})$ to obtain the deacetylated compound $(227 \mathrm{mg})$. The 
obtained product was hydrogenolysed with $\mathrm{Pd}(\mathrm{OH})_{2}-\mathrm{C}(68 \mathrm{mg})$ in $\mathrm{MeOH}(3.8 \mathrm{~mL})$ to give the corresponding amine (110 mg, $0.38 \mathrm{mmol})$. Then, the peptide coupling was carried out with the amine (110 mg), 30-D (195 mg, $0.59 \mathrm{mmol})$, DIPEA $(0.3 \mathrm{~mL}, 1.8 \mathrm{mmol})$ and HATU $(206 \mathrm{mg}, 0.54 \mathrm{mmol})$ in DMF $(7.5 \mathrm{~mL})$. The residue was purified by flash chromatography on silica gel (EtOAc/EtOH 99:1 to 92:8) to afford clean product 33 (204 mg, $0.34 \mathrm{mmol}, 47 \%)$ as a yellow powder. $[\alpha]_{\mathrm{D}}{ }^{25}+24.4(c=0.55, \mathrm{MeOH}) .{ }^{1} \mathrm{H} \mathrm{NMR}\left(500 \mathrm{MHz}, \mathrm{CD}_{3} \mathrm{OD}\right){ }^{34} \delta 7.60\left(\mathrm{~s}, 1 \mathrm{H}, H_{\mathrm{Ar}}\right), 5.55\left(\mathrm{~d}, 1 \mathrm{H}, J_{1,2}=\right.$ $9.0 \mathrm{~Hz}, H 1), 4.45\left(\mathrm{t}, 1 \mathrm{H}, J_{7,8}=J_{7,8}=5.0 \mathrm{~Hz}, H 7\right), 4.01-3.91(\mathrm{~m}, 2 \mathrm{H}, H 9), 3.89-3.55(\mathrm{~m}, 7 \mathrm{H}, H 2, H 3, H 4, H 5, H 6$, H8), 2.99-2.88 (m, 3H, NCH3), $1.91(\mathrm{~s}, 3 \mathrm{H}, \mathrm{CH} 3), 1.57-1.35\left(\mathrm{~m}, 9 \mathrm{H}, \mathrm{OC}\left(\mathrm{CH}_{3}\right) 3\right), 1.21\left(\mathrm{~s}, 9 \mathrm{H}, \mathrm{CO}_{2} \mathrm{C}\left(\mathrm{CH}_{3}\right)_{3}\right) ;{ }^{13} \mathrm{C} \mathrm{NMR}$ $\left(75 \mathrm{MHz}, \mathrm{CD}_{3} \mathrm{OD}\right)^{35} \delta 172.0(C=\mathrm{O}), 170.6(C=\mathrm{O}), 170.5(C=\mathrm{O}), 164.7(C=\mathrm{O}), 151.5(C=\mathrm{O}), 136.7\left(C \mathrm{H}_{\mathrm{Ar}}\right), 110.5$ $\left(C q_{\mathrm{Ar}}\right), 85.5(\mathrm{Cl}), 80.3\left(C\left(\mathrm{CH}_{3}\right)_{3}\right), 78.3(\mathrm{CH}), 74.0(\mathrm{CH}), 73.5\left(C\left(\mathrm{CH}_{3}\right)_{3}\right), 71.9(\mathrm{CH}), 61.4\left(\mathrm{CH}_{2}\right), 54.3(C 7), 51.7$ $\left.(\mathrm{CH}), 51.5\left(\mathrm{CH}_{2}\right), 35.1\left(\mathrm{NCH}_{3}\right), 27.2\left(\mathrm{C}\left(\mathrm{CH}_{3}\right)_{3}\right), 26.2\left(\mathrm{C}_{(\mathrm{CH}}\right)_{3}\right), 10.8\left(\mathrm{CH}_{3}\right) ; \mathrm{IR} v\left(\mathrm{film}, \mathrm{cm}^{-1}\right) 3295(\mathrm{~N}-\mathrm{H}), 2974$ $(\mathrm{CH}), 1654(\mathrm{NH}-\mathrm{C}=\mathrm{O})$; ESIHRMS $m / z=602.4783[\mathrm{M}+\mathrm{H}]^{+} . \mathrm{C}_{26} \mathrm{H}_{44} \mathrm{~N}_{5} \mathrm{O}_{11}$ requires 602.3037.

Peptidonucleoside 35. To a stirred solution of 33 (30 mg, $0.05 \mathrm{mmol}, 1$ eq.) in $\mathrm{CH}_{2} \mathrm{Cl}_{2} / \mathrm{MeOH}(2: 1 \mathrm{v} / \mathrm{v}, 0.6 \mathrm{~mL})$ was added a solution of $4 \mathrm{M} \mathrm{HCl}$ in dioxane $(0.3 \mathrm{~mL}, 1.2 \mathrm{mmol}, 25$ eq. $)$. The resulting mixture was stirred at room temperature for 2 days and then diluted with $\mathrm{H}_{2} \mathrm{O}$ and then neutralized with $\mathrm{NEt}_{3}(0.2 \mathrm{~mL})$. The mixture was concentrated under vaccum and the crude product was purified by preparative TLC $\left(\mathrm{H}_{2} \mathrm{O} / \mathrm{EtOH} / \mathrm{EtOAc} 2: 2: 1\right.$, with $1 \%$ of $\left.\mathrm{NH}_{4} \mathrm{OH}\right)$ to afford clean product 35 as a white powder $(10 \mathrm{mg}, 0.022 \mathrm{mmol}, 45 \%) ;[\alpha]_{\mathrm{D}}{ }^{20}+8.9(c=1.0, \mathrm{MeOH})$; ${ }^{1} \mathrm{H}$ NMR (300 MHz, $\left.\mathrm{D}_{2} \mathrm{O}\right) \delta 7.62\left(\mathrm{~s}, 1 \mathrm{H}, H_{\mathrm{Ar}}\right), 5.61-5.54(\mathrm{~m}, 1 \mathrm{H}, H 1), 4.44\left(\mathrm{t}, 1 \mathrm{H}, J_{7,8}=J_{7,8}=5.5 \mathrm{~Hz}, H 7\right), 3.92-3.79$ $\left(\mathrm{m}, 4 \mathrm{H}, 1 \times \mathrm{CH}\right.$ and $\left.\mathrm{CH}_{2}\right), 3.79-3.64(\mathrm{~m}, 5 \mathrm{H}, 3 \times \mathrm{CH}$ and $\mathrm{CH} 2), 3.63-3.54\left(\mathrm{~m}, 1 \mathrm{H}, \mathrm{CH}_{2}\right), 2.67(\mathrm{~s}, 3 \mathrm{H}, \mathrm{NCH}), 1.86(\mathrm{~s}$, $3 \mathrm{H}, \mathrm{CH}) ;{ }^{13} \mathrm{C}$ NMR $\left(75 \mathrm{MHz}, \mathrm{D}_{2} \mathrm{O}\right) \delta 172.1(\mathrm{C}=\mathrm{O}), 167.8(\mathrm{C}=\mathrm{O}), 166.3(\mathrm{C}=\mathrm{O}), 152.2(\mathrm{C}=\mathrm{O}), 137.3\left(\mathrm{CH}_{\mathrm{Ar}}\right), 111.1$ $\left(C q_{\mathrm{Ar}}\right), 82.5(\mathrm{Cl}), 77.5(\mathrm{CH}), 73.6(\mathrm{CH}), 71.6(\mathrm{CH}), 61.1\left(\mathrm{CH}_{2}\right), 60.7\left(\mathrm{CH}_{2}\right), 56.0(\mathrm{C7}), 51.4(\mathrm{CH}), 49.4\left(\mathrm{CH}_{2}\right), 33.1$ $\left(\mathrm{NCH}_{3}\right), 11.4\left(\mathrm{CH}_{3}\right)$; IR $v\left(\right.$ film, $\left.\mathrm{cm}^{-1}\right) 3288(\mathrm{~N}-\mathrm{H}), 2923(\mathrm{CH}), 2854(\mathrm{CH}), 1664(\mathrm{NH}-\mathrm{C}=\mathrm{O})$; ESIHRMS m/z= $446.1867[\mathrm{M}+\mathrm{H}]^{+}$. $\mathrm{C}_{18} \mathrm{H}_{27} \mathrm{~N}_{5} \mathrm{O}_{9}$ requires 446.1887 .

Peptidonucleoside 36. The same Procedure as described for 31-D was followed using 17 (194 mg, $0.72 \mathrm{mmol}$ ), solution $1(1.2 \mathrm{~mL}, \mathrm{C}=0.22 \mathrm{M}, 40 \mathrm{~mol} \%$ of $\mathrm{Na})$ in $\mathrm{MeOH}(7 \mathrm{~mL})$. The obtained product $(125 \mathrm{mg})$ was hydrogenolysed with $\mathrm{Pd}(\mathrm{OH})_{2}-\mathrm{C}(50 \mathrm{mg})$ in $\mathrm{MeOH}(4 \mathrm{~mL})$ to give the corresponding amine $(107 \mathrm{mg})$. Then, the peptide coupling was carried out using the amine (107 mg, $0.37 \mathrm{mmol}), 30-\mathbf{D}(158 \mathrm{mg}, 0.48 \mathrm{mmol})$, DIPEA $(0.25 \mathrm{~mL}, 1.48 \mathrm{mmol})$ and HATU (167 mg, $0.44 \mathrm{mmol})$ in DMF $(6 \mathrm{~mL})$. The residue was purified by chromatography on silica gel $(\mathrm{DCM} / \mathrm{MeOH}$ 96:4 to $92: 8)$ to afford $34\left(124 \mathrm{mg}, 0.20 \mathrm{mmol}, 56 \%\right.$ over three steps). ESIHRMS $\mathrm{m} / z=606.2786[\mathrm{M}+\mathrm{H}]^{+}$. $\mathrm{C}_{25} \mathrm{H}_{41} \mathrm{~N}_{5} \mathrm{O}_{11} \mathrm{~F}$ requires 606.2787 . As 34 was not very clean, it was not fully characterized and then engaged in the next step. To a stirred solution of 34 (124 mg, $0.2 \mathrm{mmol}, 1$ eq.) in $\mathrm{CH}_{2} \mathrm{Cl}_{2} / \mathrm{MeOH}(2: 1 \mathrm{v} / \mathrm{v}, 2.3 \mathrm{~mL})$ was added a solution of $4 \mathrm{M} \mathrm{HCl}$ in dioxane $(0.35 \mathrm{~mL}, 1.4 \mathrm{mmol}, 6.8$ eq. $)$. The resulting mixture was stirred at room temperature for 3 days and then diluted with $\mathrm{H}_{2} \mathrm{O}$ and then neutralized with $\mathrm{NEt}_{3}(0.2 \mathrm{~mL})$. The mixture was concentrated under vaccum and the crude product was purified by preparative TLC $\left(\mathrm{H}_{2} \mathrm{O} / \mathrm{EtOH} / \mathrm{EtOAc} 3: 1: 1\right.$, with $1 \%$ of $\left.\mathrm{NH}_{4} \mathrm{OH}\right)$ to afford clean product 36 as a white powder $(54 \mathrm{mg}, 0.013 \mathrm{mmol}, 63 \%)$. $[\alpha]_{\mathrm{D}}{ }^{20}-73.3\left(c=0.15, \mathrm{H}_{2} \mathrm{O} / \mathrm{MeOH}: 1: 1\right) ;{ }^{1} \mathrm{H}$ NMR $\left(300 \mathrm{MHz}, \mathrm{D}_{2} \mathrm{O}\right) \delta 7.94\left(\mathrm{~d}, 1 \mathrm{H}, J_{H A r, F}=6.0 \mathrm{~Hz}, H_{\mathrm{Ar}}\right), 5.59\left(\mathrm{~d}, 1 \mathrm{H}, J_{l, 2}=9.0 \mathrm{~Hz}, H 1\right), 4.51-4.38(\mathrm{~m}, 1 \mathrm{H}, H 7)$, 3.99-3.46 (m, 10H, H2, H3, H4, H5, H6, H8, H9), $2.72(\mathrm{~s}, 3 \mathrm{H}, \mathrm{NCH}), 2.72\left(\mathrm{~s}, 9 \mathrm{H}, \mathrm{C}\left(\mathrm{CH}_{3}\right)_{3}\right) ;{ }^{13} \mathrm{C} \mathrm{NMR}(75 \mathrm{MHz}$, $\left.\mathrm{D}_{2} \mathrm{O}\right) \delta 181.4(C=\mathrm{O}), 172.3(C=\mathrm{O}), 166.7(C=\mathrm{O}), 161.2\left(\mathrm{~d}, J=23.6 \mathrm{~Hz}, C \mathrm{H}_{\mathrm{Ar}}\right), 152.2(\mathrm{CO}), 125.3(\mathrm{~d}, J=23.6 \mathrm{~Hz}$, $C q A r), 125.2(\mathrm{CH}), 82.9(\mathrm{C1}), 77.5(\mathrm{CH}), 75.0\left(\mathrm{C}\left(\mathrm{CH}_{3}\right)_{3}\right), 73.3(\mathrm{CH}), 71.7(\mathrm{CH}), 61.0\left(\mathrm{CH}_{2}\right), 60.7\left(\mathrm{CH}_{2}\right), 56.0(\mathrm{C} 7)$,

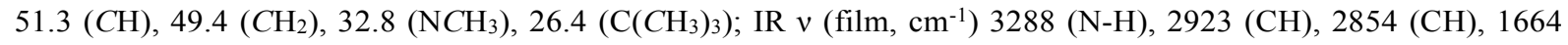
$(\mathrm{NH}-\mathrm{C}=\mathrm{O})$; ESIHRMS $m / z=506.2249[\mathrm{M}+\mathrm{H}]^{+} . \mathrm{C}_{20} \mathrm{H}_{33} \mathrm{~N}_{5} \mathrm{O} 9 \mathrm{~F}$ requires 506.2262.

tert-butyl (R)-(2-((1-(tert-butoxy)-3-hydroxypropan-2-yl)amino)-2-oxoethyl)(methyl)carbamate 37. To a stirred solution of 29-D (193 mg, $0.557 \mathrm{mmol}, 1$ eq.) in THF (2 mL) was added at $0{ }^{\circ} \mathrm{C} \mathrm{LiBH}_{4}(21 \mathrm{mg}, 0.95 \mathrm{mmol}, 1.7 \mathrm{eq}$.). The resulting mixture was stirred at room temperature for $4 \mathrm{~h}$ and then hydrolyzed with a saturated aqueous solution of $\mathrm{NH}_{4} \mathrm{Cl}(10 \mathrm{~mL})$. Water $(10 \mathrm{~mL})$ was added and the aqueous phase was extracted with EtOAc $(3 \mathrm{x} 15 \mathrm{~mL})$. The organic phase was dried with $\mathrm{Na}_{2} \mathrm{SO}_{4}$, filtered and concentrated under vacuum. The crude product was purified by flash chromatography on silica gel (Heptane/EtOAc 30:70 to 0:100) to afford product 37 (160 mg, $0.5 \mathrm{mmol}, 90 \%$ ) as a colorless oil. $[\alpha]_{\mathrm{D}}{ }^{20}-5.4\left(c=0.24, \mathrm{CHCl}_{3}\right) ;{ }^{1} \mathrm{H} \mathrm{NMR}\left(300 \mathrm{MHz}, \mathrm{CDCl}_{3}\right)^{34} \delta 6.81-6.58(\mathrm{bs}, 1 \mathrm{H}, \mathrm{NH}), 4.03-3.93$ $(\mathrm{m}, 1 \mathrm{H}, \mathrm{CHN}), 3.91-3.81\left(\mathrm{~m}, 2 \mathrm{H}, \mathrm{COCH}_{2} \mathrm{~N}\right), 3.80\left(\mathrm{dd}, 1 \mathrm{H}, J=11.5\right.$ and $\left.3.5 \mathrm{~Hz}, \mathrm{CH}_{2}\right), 3.69-3.58\left(\mathrm{~m}, 1 \mathrm{H}, \mathrm{CH}_{2}\right), 3.57-$ $3.50(\mathrm{~m}, 2 \mathrm{H}, \mathrm{CH} 2), 3.25-3.09(\mathrm{~m}, 1 \mathrm{H}, \mathrm{OH}), 2.91(\mathrm{~s}, 3 \mathrm{H}, \mathrm{NCH}), 1.43\left(\mathrm{~s}, 9 \mathrm{H}, \mathrm{C}\left(\mathrm{CH}_{3}\right)_{3}\right), 1.15\left(\mathrm{~s}, 9 \mathrm{H}, \mathrm{C}\left(\mathrm{CH}_{3}\right)_{3}\right) ;{ }^{13} \mathrm{C}$

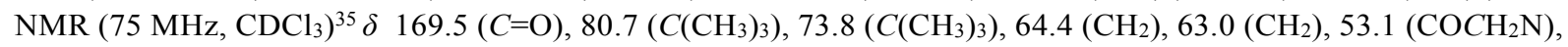
$50.5(\mathrm{CHN}), 35.6\left(\mathrm{NCH}_{3}\right), 28.3\left(\mathrm{C}\left(\mathrm{CH}_{3}\right)_{3}\right), 27.3\left(\mathrm{C}_{\left.\left(\mathrm{CH}_{3}\right)_{3}\right)}\right)$ IR $v\left(\right.$ film, cm $\left.{ }^{-1}\right) 3217(\mathrm{~N}-\mathrm{H}), 2974(\mathrm{CH}), 2875(\mathrm{CH})$, $1662(\mathrm{NH}-\mathrm{C}=\mathrm{O})$; ESIHRMS $m / z=419.2219[\mathrm{M}+\mathrm{H}]^{+}$. $\mathrm{C}_{15} \mathrm{H}_{31} \mathrm{~N}_{2} \mathrm{O}_{5}$ requires 419.2233.

tert-butyl (R)-(2-((1-(tert-butoxy)but-3-yn-2-yl)amino)-2-oxoethyl)(methyl)carbamate 38. To a stirred solution of alcohol 37 (0.130 g, $0.408 \mathrm{mmol}, 1.0$ eq.) in $\mathrm{CH}_{2} \mathrm{Cl}_{2}(8 \mathrm{~mL})$ at $0{ }^{\circ} \mathrm{C}$ was added saturated aqueous $\mathrm{NaHCO}_{3}(4 \mathrm{~mL})$, 
$\mathrm{KBr}$ (49 mg, $0.408 \mathrm{mmol}, 1.0$ eq.) and TEMPO (3 mg, $0.02 \mathrm{mmol}, 0.05$ eq.). $\mathrm{NaOCl}$ (0.5 M, $1.6 \mathrm{~mL}, 2.0$ eq.) was then added with a syringe pump over $30 \mathrm{~min}$. Saturated aqueous $\mathrm{Na}_{2} \mathrm{~S}_{2} \mathrm{O}_{3}(4 \mathrm{~mL})$ was added, the phases were separated and the aqueous layer was extracted with DCM $(3 \times 15 \mathrm{~mL})$. The combined organic layers were dried over $\mathrm{Na}_{2} \mathrm{SO}_{4}$, filtered and concentrated under reduced pressure to afford the corresponding crude $\alpha$-amino aldehyde ( $95 \mathrm{mg}$ ) which was used directly in the next step. To the latter $(90 \mathrm{mg}, 0.284 \mathrm{mmol}, 1$ eq.) and dimethyl (1-diazo-2oxopropyl)phosphonate ( $96 \mathrm{mg}, 0.498 \mathrm{mmol}, 1.75$ eq.) in $\mathrm{MeOH}(2 \mathrm{~mL})$ at $0{ }^{\circ} \mathrm{C}$ was added $\mathrm{K}_{2} \mathrm{CO}_{3}(83 \mathrm{mg}, 0.597$ mmol, 2.1 eq.). The resulting mixture was stirred at $0{ }^{\circ} \mathrm{C}$ for $3 \mathrm{~h}$ and was hydrolyzed with a sat. aq. solution of $\mathrm{NH}_{4} \mathrm{Cl}$ $(10 \mathrm{~mL})$. Water $(10 \mathrm{~mL})$ was added and the aqueous phase was extracted with EtOAc $(3 \times 15 \mathrm{~mL})$. The organic phase was dried with $\mathrm{Na}_{2} \mathrm{SO}_{4}$, filtered and concentrated under vacuum pressure. The crude product was purified by flash chromatography on silica gel (Heptane/EtOAc 70:30 to 50:50) to afford product 38 (51 mg, 0.163 mmol, 42\% over the two steps) as a colorless oil. $[\alpha]_{\mathrm{D}}{ }^{20}+2.5\left(c=0.32, \mathrm{CHCl}_{3}\right) ;{ }^{1} \mathrm{H}$ NMR $\left(300 \mathrm{MHz}, \mathrm{CDCl}_{3}\right)^{34} \delta 6.65-6.26(\mathrm{bs}, 1 \mathrm{H}$, $\mathrm{NH}), 4.83-4.69(\mathrm{~m}, 1 \mathrm{H}, \mathrm{CHN}), 3.90-3.67\left(\mathrm{~m}, 2 \mathrm{H}, \mathrm{COCH}_{2} \mathrm{~N}\right), 3.50-3.37\left(\mathrm{~m}, 2 \mathrm{H}, \mathrm{CH}_{2}\right), 2.86\left(\mathrm{~s}, 3 \mathrm{H}, \mathrm{CH}_{3}\right), 2.17(\mathrm{~d}, 1 \mathrm{H}$, $J=2.5 \mathrm{~Hz}, \mathrm{CH}$ Alkyne), $1.41\left(\mathrm{~s}, 9 \mathrm{H}, \mathrm{C}\left(\mathrm{CH}_{3}\right)_{3}\right), 1.09 \mathrm{~s}, 9 \mathrm{H},\left(\mathrm{C}\left(\mathrm{CH}_{3}\right)_{3}\right) ;{ }^{13} \mathrm{C} \mathrm{NMR}\left(75 \mathrm{MHz}, \mathrm{CDCl}_{3}\right)^{35} \delta 168.5(\mathrm{C}=\mathrm{O})$, 81.4 (CqAlkyne), $80.7\left(C\left(\mathrm{CH}_{3}\right)_{3}\right), 73.6\left(C\left(\mathrm{CH}_{3}\right)_{3}\right), 70.8\left(\mathrm{CH}_{\text {Alkyne }}\right), 63.5\left(\mathrm{CH}_{2}\right), 53.1\left(\mathrm{CH}_{2}\right), 41.6\left(\mathrm{COCH} \mathrm{H}_{2}\right), 35.6$

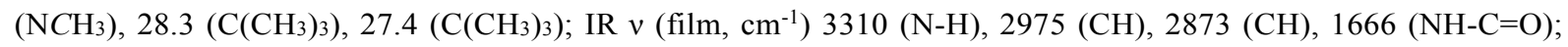
ESIHRMS $m / z=335.1947[\mathrm{M}+\mathrm{Na}]^{+}$. $\mathrm{C}_{16} \mathrm{H}_{28} \mathrm{~N}_{2} \mathrm{O}_{4}$ requires 335.1947.

Peptidonucleoside 39. To a stirred solution of azid 18 (78 mg, $0.144 \mathrm{mmol}, 1.0$ eq.) and alkyne 38 (45 mg, 0.144 mmol, 1.0 eq.) in $\mathrm{CH}_{2} \mathrm{Cl}_{2}(2 \mathrm{~mL})$ at r.t. was added $\mathrm{CuSO}_{4} \cdot 5 \mathrm{H}_{2} \mathrm{O}(4 \mathrm{mg}, 0.014 \mathrm{mmol}, 0.1$ eq. $)$ in water ( $\left.1 \mathrm{~mL}\right)$ followed by sodium ascorbate $(3 \mathrm{mg}, 0.014 \mathrm{mmol}, 0.1$ eq.) in water $(1 \mathrm{~mL})$. After stirring for $18 \mathrm{~h}$, water $(10 \mathrm{~mL})$ and EtOAc $(15 \mathrm{~mL})$ were added. The phases were separated and the aqueous layer was extracted with EtOAc $(3 \times 15 \mathrm{~mL})$. The combined organic layers were dried over $\mathrm{Na}_{2} \mathrm{SO}_{4}$, filtered and concentrated under reduced pressure. The crude product was purified by flash chromatography on silica gel (Heptane/EtOAc 50:50 to 0:100) to afford product 39 (94 mg, $0.163 \mathrm{mmol}, 76 \%)$ as a colorless oil. $[\alpha]_{\mathrm{D}}{ }^{20}-26.9\left(c=0.58, \mathrm{CHCl}_{3}\right) ;{ }^{1} \mathrm{H} \mathrm{NMR}\left(300 \mathrm{MHz}, \mathrm{CDCl}_{3}\right)^{34} \delta 8.28(\mathrm{~d}, 2 \mathrm{H}, J$ $\left.=7.5 \mathrm{~Hz}, H_{\mathrm{Ar}}\right), 7.57\left(\mathrm{~s}, 1 \mathrm{H}, H_{\text {triazole }}\right), 7.54-7.46\left(\mathrm{~m}, 1 \mathrm{H}, H_{\mathrm{Ar}}\right), 7.42\left(\mathrm{t}, 2 \mathrm{H}, J=7.5 \mathrm{~Hz}, H_{\mathrm{Ar}}\right), 7.27\left(\mathrm{~s}, 1 \mathrm{H}, H_{\mathrm{Ar}}\right), 6.94-6.77$ (bs, $1 \mathrm{H}, \mathrm{NH}), 6.09\left(\mathrm{~d}, 1 \mathrm{H}, J_{1,2}=9.5 \mathrm{~Hz}, H 1\right), 5.79\left(\mathrm{t}, 1 \mathrm{H}, J_{3,4}=J_{3,2}=9.5 \mathrm{~Hz}, H 3\right), 5.27\left(\mathrm{t}, 1 \mathrm{H}, J_{2,3}=J_{2,1}=9.5 \mathrm{~Hz}, H 2\right)$, $5.23-5.12(\mathrm{~m}, 1 \mathrm{H}, \mathrm{CHN}), 4.73\left(\mathrm{t}, 1 \mathrm{H}, J_{4,5}=J_{4,3}=9.5 \mathrm{~Hz}, H 4\right), 4.62-4.61(\mathrm{~m}, 1 \mathrm{H}, H 5), 4.08(\mathrm{dd}, 1 \mathrm{H}, J=2.0$ and 12.0 $\left.\mathrm{Hz}, \mathrm{CH}_{2}\right), 3.94-3.73\left(\mathrm{~m}, 4 \mathrm{H}, \mathrm{CH}_{2}\right), 3.62-3.49\left(\mathrm{~m}, 1 \mathrm{H}, \mathrm{CH}_{2}\right), 2.92\left(\mathrm{~s}, 3 \mathrm{H}, \mathrm{NCH}_{3}\right), 2.12(\mathrm{~s}, 3 \mathrm{H}, \mathrm{CH}), 2.04(\mathrm{~s}, 3 \mathrm{H}$, $\left.\mathrm{COCH}_{3}\right), 1.97\left(\mathrm{~s}, 3 \mathrm{H}, \mathrm{COCH}_{3}\right), 1.85\left(\mathrm{~s}, 3 \mathrm{H}, \mathrm{COCH}_{3}\right), 1.45\left(\mathrm{~s}, 9 \mathrm{H}, \mathrm{C}\left(\mathrm{CH}_{3}\right)_{3}\right), 1.09\left(\mathrm{~s}, 9 \mathrm{H}, \mathrm{C}\left(\mathrm{CH}_{3}\right)_{3}\right) ;{ }^{13} \mathrm{C} \mathrm{NMR}(75$ $\left.\mathrm{MHz}, \mathrm{CDCl}_{3}\right)^{35} \delta 179.9(C=\mathrm{O}), 169.9(C=\mathrm{O}), 169.7(C=\mathrm{O}), 169.0(C=\mathrm{O}), 168.5(C=\mathrm{O}), 158.8(C=\mathrm{O}), 148.0(C \mathrm{q})$, $136.7(C \mathrm{q}), 135.1\left(C \mathrm{H}_{\mathrm{Ar}}\right), 132.8\left(C \mathrm{H}_{\mathrm{Ar}}\right), 130.0\left(C \mathrm{H}_{\mathrm{Ar}}\right), 128.1\left(C \mathrm{H}_{\mathrm{Ar}}\right), 122.6\left(C \mathrm{H}_{\text {Triazole }}\right), 113.2\left(C \mathrm{H}_{\mathrm{Ar}}\right), 80.6(C 1), 77.2$ $(C q), 74.9(C 5), 73.5(C q), 72.2(C 3), 69.7(C 2), 62.8\left(\mathrm{CH}_{2}\right), 61.8\left(\mathrm{CH}_{2}\right), 59.9(C 4), 53.0\left(\mathrm{CH}_{2}\right), 46.5(C \mathrm{HN}), 35.8$

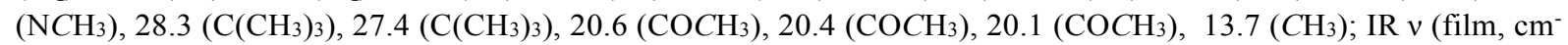
$\left.{ }^{1}\right) 2973(\mathrm{CH}), 2854(\mathrm{CH}), 1750(\mathrm{CO}), 1704(\mathrm{CO}), 1659(\mathrm{NH}-\mathrm{C}=\mathrm{O})$; ESIHRMS $m / z=855.3889[\mathrm{M}+\mathrm{H}]^{+} . \mathrm{C}_{40} \mathrm{H}_{55} \mathrm{~N}_{8} \mathrm{O}_{13}$ requires 855.13889 .

Peptidonucleoside 40. The solution 1 was prepared with $\mathrm{Na}(20 \mathrm{mg})$ in dry $\mathrm{MeOH}(2 \mathrm{~mL})$. To a stirred solution of the protected nucleoside $39(70 \mathrm{mg}, 0.028 \mathrm{mmol})$ in dry $\mathrm{MeOH}(0.2 \mathrm{~mL})$ was added the solution $1(0.4 \mathrm{~mL}, 1 \mathrm{eq}$.$) .$ The resulting mixture was stirred at room temperature for $5 \mathrm{~h}$ and a solution of $4 \mathrm{M} \mathrm{HCl}$ in dioxane $(0.6 \mathrm{~mL}, 2.4 \mathrm{mmol}$, 30 eq.) was added. The resulting mixture was stirred at room temperature for $8 \mathrm{~h}$ and neutralized with $\mathrm{NEt}_{3}(0.35 \mathrm{~mL})$. The mixture was concentrated under vaccum and the crude product was purified by preparative TLC $\left(\mathrm{H}_{2} \mathrm{O} / \mathrm{EtOH} / \mathrm{EtOAc} 1: 2: 2\right.$, with $1 \%$ of $\left.\mathrm{NH}_{4} \mathrm{OH}\right)$ to afford product $\mathbf{4 0}$ as a white powder, which was washed several times with $\mathrm{CHCl}_{3}(25 \mathrm{mg}, 0.047 \mathrm{mmol}, 58 \%)$. $[\alpha]_{\mathrm{D}}{ }^{20}-43.4\left(c=0.35, \mathrm{H}_{2} \mathrm{O} / \mathrm{MeOH}: 1 / 1\right) ;{ }^{1} \mathrm{H} \mathrm{NMR}\left(300 \mathrm{MHz}, \mathrm{CD}{ }_{3} \mathrm{OD}\right)$ $\delta 8.15\left(\mathrm{~s}, 1 \mathrm{H}, H_{\text {triazole }}\right), 7.91\left(\mathrm{~s}, 1 \mathrm{H}, H_{\mathrm{Ar}}\right), 7.62-7.24(\mathrm{bs}, 1 \mathrm{H}, \mathrm{NH}), 5.89\left(\mathrm{~d}, 1 \mathrm{H}, J_{l, 2}=9.5 \mathrm{~Hz}, H 1\right), 5.31(\mathrm{t}, 1 \mathrm{H}, J=5.5$ $\mathrm{Hz}, \mathrm{C} H \mathrm{~N}), 4.74\left(\mathrm{t}, 1 \mathrm{H}, J_{3,4}=J_{3,2}=9.5 \mathrm{~Hz}, H 3\right), 5.34-4.24(\mathrm{~m}, 2 \mathrm{H}, H 4$ and $H 5), 3.97-3.88(\mathrm{~m}, 2 \mathrm{H}, \mathrm{CH}), 3.85(\mathrm{t}, 1 \mathrm{H}$, $\left.J_{2,1}=J_{2,3}=9.5 \mathrm{~Hz}, H 2\right), 3.82-3.71(\mathrm{~m}, 2 \mathrm{H}, \mathrm{CH}), 3.52(\mathrm{dd}, 1 \mathrm{H}, J=1.5$ and $12.0 \mathrm{~Hz}, H 6), 3.52(\mathrm{dd}, 1 \mathrm{H}, J=4.0$ and 12.0

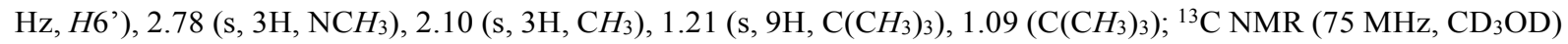
$\delta 164.9(C=\mathrm{O}), 163.4(C=\mathrm{O}), 153.5(C \mathrm{q}), 145.8(C \mathrm{q}), 140.6\left(\mathrm{CH}_{\mathrm{Ar}}\right), 124.0\left(C \mathrm{H}_{\text {Triazole }}\right), 100.7(C \mathrm{q}), 83.7(C 1), 77.5$ and $74.4(C 4$ and $C 5), 73.4(C q), 72.4(C 2), 63.0\left(\mathrm{CH}_{2}\right), 61.7(\mathrm{C3}), 60.0(\mathrm{C6}), 49.4\left(\mathrm{CH}_{2}\right), 47.2(\mathrm{CHN}), 32.4\left(\mathrm{NCH}_{3}\right)$, $26.3\left(\mathrm{C}\left(\mathrm{CH}_{3}\right)_{3}\right), 11.7\left(\mathrm{CH}_{3}\right)$; IR $v\left(\right.$ film, $\left.\mathrm{cm}^{-1}\right) 3056(\mathrm{NH}), 2921(\mathrm{CH}), 1666(\mathrm{NH}-\mathrm{C}=\mathrm{O})$; ESIHRMS $\mathrm{m} / z=525.2787$ $[\mathrm{M}+\mathrm{H}]^{+}$. $\mathrm{C}_{22} \mathrm{H}_{37} \mathrm{~N}_{8} \mathrm{O}_{7}$ requires 525.2785.

\section{Conflicts of interest}

There are no conflicts to declare

\section{Acknowledgements}

We gratefully acknowledge financial supports from CNRS and from Bayer CropScience (PhD thesis of MB). 


\section{Notes and references}

1 R. P. Oliver and H. G. Hewitt, Fungicides in crop protection, Cabi, 2014.

P. Jeschke, Pest Manag Sci, 2016, 72, 210.

3 M. C. Fisher, N. J. Hawkins, D. Sanglard and S. J. Gurr, Science, 2018, 360, 739.

4 T. Kanzaki, E. Higashide, H. Yamamoto, H. Shibata, K. Nakazma, H. Iwasaki, T. Takewaka, A. Miyake, J. Antibiot. (Tokyo), 1962, 15, 93

5 W. Andersch, R. N. Royalty, F. D. Smith, B. Springer and W. Thielert, Compositions comprising gougerotin and a biological control agent, US20150373973A1.

6 a) K. A. Watanabe, E. A. Falco and J. J. Fox, J. Am. Chem. Soc., 1972, 94, 3272. b) F. W. Lichtenthaler, T. Morino, W. Winterfeldt and Y. Sanemitsu, Tetrahedron Lett., 1975, 16, 3527.

7 M. T. Migawa, L. M. Risen, R. H. Griffey and E. E. Swayze, Organic Letters, 2005, 7, 3429.

8 L. Thiry, J. Gen. Vir., 1968, 2, 143.

9 J. M. Clark and J. K. Gunther, Biochim. Biophys. Acta, 1963, 76, 636.

10 A. R. Burkett, K. K. Schlender and H. M. Sell, Phytochemistry, 1970, 9, 545.

11 a) U. Niedballa and H. Vorbrüggen, Angew. Chem. Int. Ed. Engl., 1970, 9, 461. b) Comprehensive Organic Name Reactions and Reagents, 2010, 2915.

12 M. Sakagami and H. Hamana, Tetrahedron Lett., 2000, 41, 5547.

13 H. Vorbrüggen and K. Krolikiewicz, Angew. Chem., Int. Ed., 1975, 14, 421.

14 U. Niedballa and H. Vorbrüggen, Angew. Chem., Int. Ed., 1970, 9, 461.

15 N. Tzioumaki, S. Manta, E. Tsoukala, J. Vande Voorde, S. Liekens, D. Komiotis and J. Balzarini, Eur J Med Chem, $2011,46,993$.

16 Q. Zhang, J. Sun, Y. Zhu, F. Zhang and B. Yu, Angew. Chem. Int. Ed. Engl., 2011, 50, 4933.

17 J. Zeng, Y. Liu, W. Chen, X. Zhao, L. Meng and Q. Wan, Top. Curr. Chem., 2018, 376, 27.

18 a) S. Norsikian, C. Tresse, M. François-Eude, L. Jeanne-Julien, G. Masson, V. Servajean, G. Genta-Jouve, J. M. Beau and E. Roulland, Angew. Chem, Int. Ed., 2020, 59, 6612. b) C. Tresse, M. François-Heude, V. Servajean, R. Ravinder, C. Lesieur, L. Geiben, L. Jeanne-Julien, V. Steinmetz, P. Retailleau, E. Roulland, J.-M. Beau and S. Norsikian, Chem. Eur. J., 2021, doi.org/10.1002/chem.202005102

19 a) D. D'Alonzo, A. Guaragna, A. Van Aerschot, P. Herdewijn and G. Palumbo, J. Org. Chem., 2010, 75, 6402. b) N. Bomholt, P. T. Jorgensen and E. B. Pedersen, Bioorg. Med. Chem. Lett., 2011, 21, 7376. c) L. Chanteloup and J.-M. Beau, Tetrahedron Lett., 1992, 33, 5347.

20 A.-T. Tran, R. A. Jones, J. Pastor, J. Boisson, N. Smith and M. C. Galan, Adv. Synth. Catal., 2011, 353, 2593.

21 A. Brar and Y. D. Vankar, Tetrahedron Lett., 2006, 47, 5207.

22 S. Liu and K. J. Edgar, Biomacromolecules, 2015, 16, 2556.

23 L. A. Carpino, H. Imazumi, B. M. Foxman, M. J. Vela, P. Henklein, A. El-Faham, J. Klose and M. Bienert, Org. Lett., 2000, 2, 2253

24 S. Norsikian, M. Beretta, A. Cannillo, A. Martin, P. Retailleau and J.-M. Beau, Chem. Commun., 2015, 51, 9991.

25 Prepared according to J. Pietruszka and A. Witt, Synthesis, 2006, 4266 using tosylazide.

26 For recent reviews see : a) V. K. Tiwari, B. B. Mishra, K. B. Mishra, N. Mishra, A. S. Singh and X. Chen, Chem. Rev., 2016, 116, 3086. b) S. Neumann, M. Biewend, S. Rana and W. H. Binder, Macromol. Rapid Commun., 2020, 41, e1900359.

27 Tamiya, M.; Ohmori, K.; Kitamura, M.; Kato, H.; Arai, T.; Oorui, M.; Suzuki, K. Chem. Eur. J. 2007, 13, 97919823.

28 Lei, Z.; Wang, J.; Mao, G.; Wen, Y.; Tian, Y.; Wu, H.; Li, Y.; Xu, H. J. Agric. Food Chem. 2014, 62, 6065-6071.

29 Tiwari, P. ; Misra, A. K. Carbohydr. Res. 2006, 341, 339-350

30 Dara, S.; Saikam, V.; Yadav, M.; Singh, P. P.; Vishwakarma, R. A. Carbohydr. Res. 2014, 391, 93-96.

31 Shalom, Y. ; Blum, J. ; Harvey, R. G. J. Heterocycl. Chem 1996, 33, 681.

32 Buchini, S. ; Leumann, C. J. Eur. J. Org. Chem. 2006, 2006, 3152-3168

33 Caso, M. F. ; D’Alonzo, D. ; D’Errico, S. ; Palumbo, G. Guaragna, A. Org. Lett. 2015, 17, 2626-2629

34 Peaks in ${ }^{1} \mathrm{H}-\mathrm{NMR}$ spectrum broad and split due to the presence of $N$-Boc rotamers

35 Peaks in ${ }^{13} \mathrm{C}$-NMR spectrum broad and split due to the presence of $N$-Boc rotamers. 